\title{
Numerical study of extreme-ultra-violet generated plasmas in hydrogen
}

Dmitry Astakhov
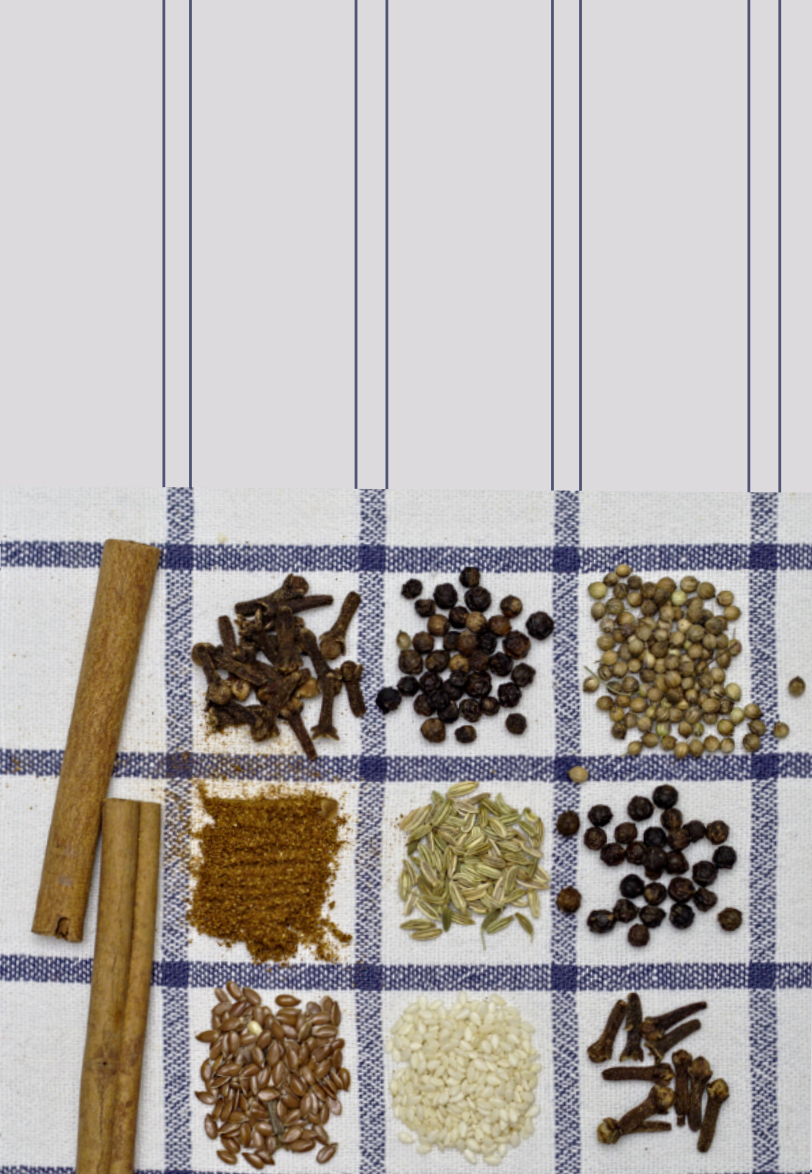


\section{NUMERICAL STUDY OF EXTREME-ULTRA-VIOLET GENERATED PLASMAS IN HYDROGEN}

\section{DISSERTATION}

to obtain

the degree of doctor at the University of Twente, on the authority of the rector magnificus, Prof.dr. H. Brinksma, on account of the decision of the graduation committee, to be publicly defended on Thursday 28 April, 2016 at 12:45

by

\section{Dmitry Astakhov}

born on 9 January 1986

in Sverdlovsk, the Russian Federation 
This dissertation has been approved by:

Promotor:

Prof.dr. F. Bijkerk, University of Twente

Co-promotor:

Dr. W. Goedheer, Utrecht University

Co-promotor:

Dr. D. Lopaev, Moscow State University/Skobeltsyn Institute of Nuclear Physics

Committee members:

Prof.dr. V. Banine, Eindhoven University of Technology

Prof.dr. U. Ebert, University of Amsterdam/Centrum Wiskunde en Informatica

Prof.dr. P.J. Kelly, University of Twente

Prof.dr. K.-J. Boller, University of Twente 
Thesis is based on the following publications:

\section{Chapter 5}

D.I. Astakhov, W.J. Goedheer, C.J. Lee, V.V. Ivanov, V.M. Krivtsun, A.I. Zotovich, S.M. Zyryanov, D.V. Lopaev, and F. Bijkerk, Plasma probe characteristics in low density hydrogen pulsed plasmas, Plasma Sources Sci. Technol. 24, 055018 (2015).

\section{Chapter 6}

D.I. Astakhov, W.J. Goedheer, C.J. Lee, V.V. Ivanov, V.M. Krivtsun, K.N. Koshelev, D.V. Lopaev, R.M. van der Horst, J. Beckers, E.A. Osorio, F. Bijkerk, Exploring the electron density in plasma induced by EUV radiation: II. Numerical studies in argon and hydrogen, submitted

\section{Chapter 7}

D.I. Astakhov, W.J. Goedheer, C.J. Lee, V.V. Ivanov, V.M. Krivtsun, O. Yakushev, K.N. Koshelev, D. V. Lopaev, and F. Bijkerk, Numerical and experimental studies of the carbon etching in EUV-induced plasma, submitted

This work is part of the research programme 'Controlling photon and plasma induced processes at EUV optical surfaces (CP3E)' of the 'Stichting voor Fundamenteel Onderzoek der Materie (FOM)', which is financially supported by the 'Nederlandse Organisatie voor Wetenschappelijk Onderzoek (NWO)'. The CP3E programme is co-financed by Carl Zeiss SMT and ASML. We also acknowledge financial support from Agentschap NL (EXEPT project).

ISBN: 978-90-365-4111-4

DOI: $10.3990 / 1.9789036541114$

CDmitry Astakhov, 2016. 

1 Introduction $\quad 13$

1.1 EUV lithography, mirrors and pulsed plasmas . . . . . . . . . . . . . . . . 13

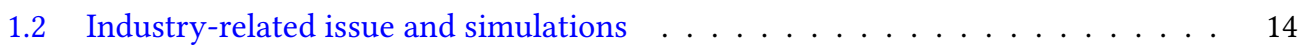

1.3 "Why a new code" . . . . . . . . . . . . . . . . 16

$2 \quad$ Numerical tool $\quad 19$

2.1 Plasma equations . . . . . . . . . . . . . . . . . . . . 19

2.1.1 Energy conserving scheme vs momentum conserving scheme . . . . . 22

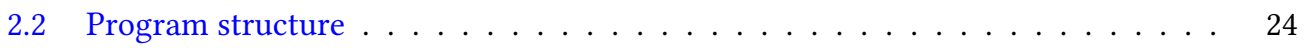

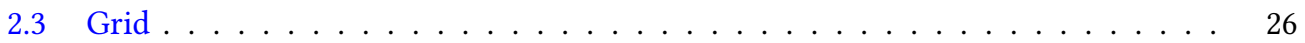

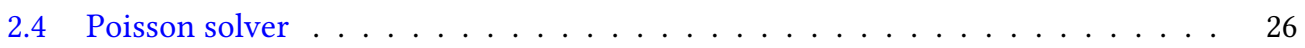

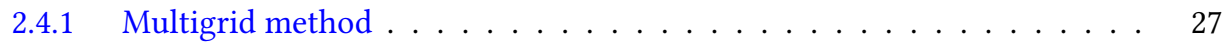

2.4 .2 Geometrical multigrid . . . . . . . . . . . . . . . . . . . . . . . . . . . . . . . . 30

2.4 .3 Algebraic multigrid . . . . . . . . . . . . . . . . . . . . . . . 32

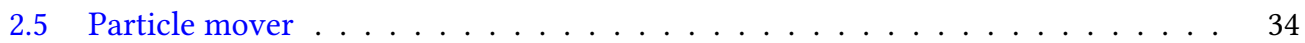

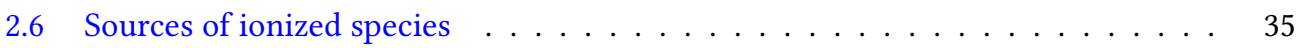

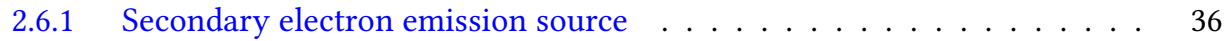

2.6 .2 Particle source . . . . . . . . . . . . . . . . 37

2.7 Collisions with background gas $\ldots \ldots \ldots \ldots \ldots \ldots \ldots \ldots$

2.8 Cross-section set and swarm parameters . . . . . . . . . . . . . . . 39

2.8 .1 Swarm experiment . . . . . . . . . . . . . . . . 40

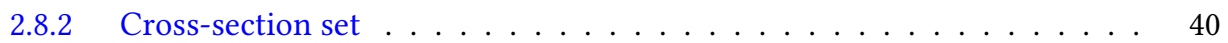

2.8 .3 Differential cross-sections . . . . . . . . . . . . . . . . . 43

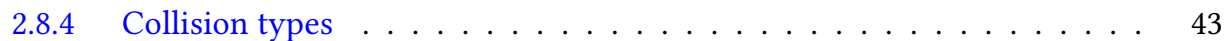

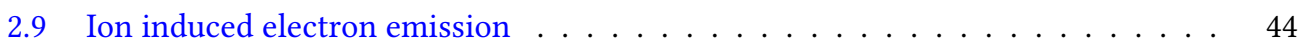

2.10 Displacement current . . . . . . . . . . . . . . . . . . . 45

3 Test problems $\quad 47$

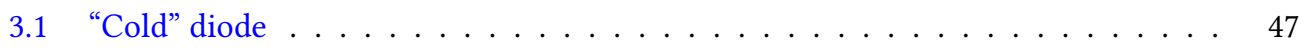

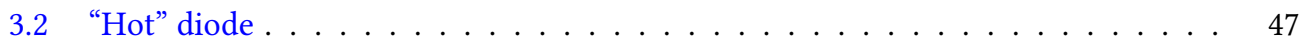

4 Research goal and experiments selection $\quad 51$

5 Plasma probe characteristics in low density hydrogen pulsed plasmas 53

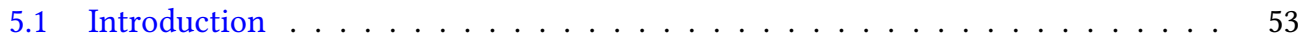

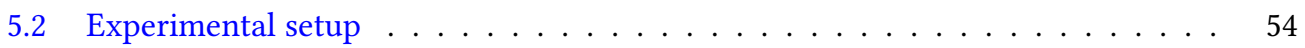

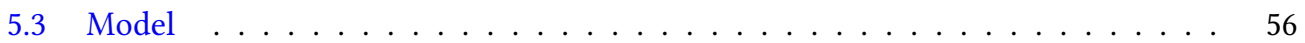

5.4 Results and discussion . . . . . . . . . . . . . . . . . . 60

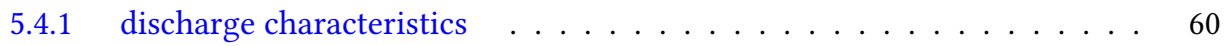

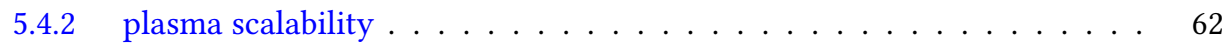

5.4.3 Axially symmetric plasma probe . . . . . . . . . . . . 62

5.4.4 Comparison between computed plasma density and that derived from measured probe characteristic . . . . . . . . . . . . . . 64

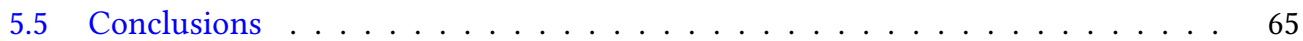


6 Exploring the electron density in plasma induced by EUV radiation:

$\begin{array}{ll}\text { II. Numerical studies in argon and hydrogen } & 71\end{array}$

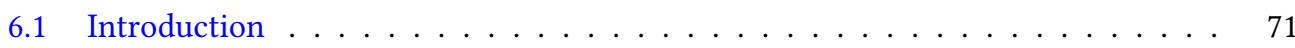

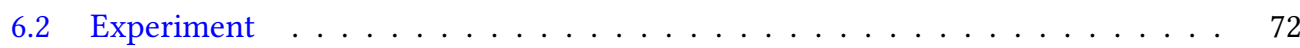

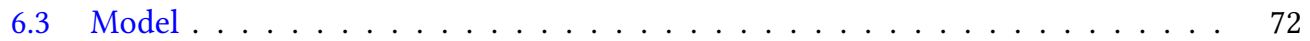

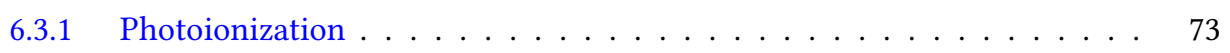

6.3 .2 EUV the spectrum ....................... 74

6.3.3 Secondary electron emission and influence of chamber configuration . $\quad 75$

6.3 .4 Grid resolution . . . . . . . . . . . . . . . . . . . 76

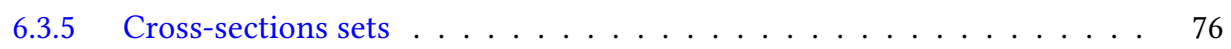

6.3.6 Calculation of the field average electron density . . . . . . . . . 78

6.4 Results and discussion . . . . . . . . . . . . . . . . . 78

6.4.1 Relation of field averaged electron density and simulated values . . . . 79

6.4.2 Effect of VUV part of the spectrum . . . . . . . . . . . . . 80

6.4.3 Effect of secondary electron emission due to EUV radiation . . . . . . . 80

6.4.4 Influence of electron induced secondary emission from cavity walls . . . 82

6.4.5 High charge imbalance plasma for $1 \mathrm{~Pa} \mathrm{H}_{2} \ldots \ldots \ldots$

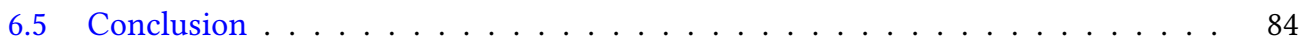

7 Numerical and experimental studies of the carbon etching in EUV-induced plasma 87

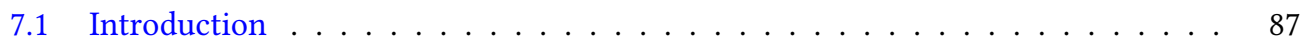

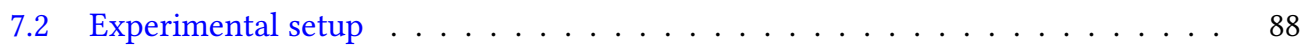

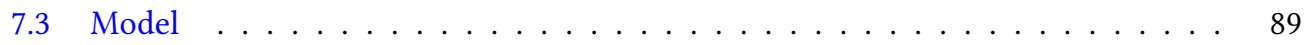

7.3 .1 Chamber configuration . . . . . . . . . . . . . . . . . . 89

7.3 .2 Deielectric model . . . . . . . . . . . . . . . . . . . . . 90

7.3.3 Length scales and grid resolution . . . . . . . . . . . . . . . . . . . . . . 90

7.3.4 photo-electron emission . . . . . . . . . . . . . . . . . . . . . . . . . . 91

7.3.5 EUV spectrum and photoionization . . . . . . . . . . . . . . . . . . . 91

7.3.6 Cross-sections set . . . . . . . . . . . . . . . . . . . . . 92

7.4 Analysis of the charge - bias characteristic . . . . . . . . . . . . . . 93

7.4.1 Average secondary electron yield . . . . . . . . . . . . . . . . . 94

7.4.2 Role of dielectric ring . . . . . . . . . . . . . . . . . . . . . . . . . . . . . . . 94

7.5 Ion fluxes to the sample surface $\ldots \ldots \ldots \ldots$

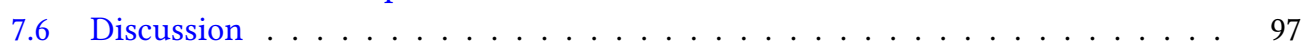

7.7 Conclusion . . . . . . . . . . . . . . . . . . . 99

8 Valorisation and outline $\quad 103$

8.1 Outlook and applications beyond EUV-induced plasmas . . . . . . . . . . . . 103

9 Conclusions $\quad 107$

$\begin{array}{lr}\text { Aknowledgments } & 109\end{array}$

$\begin{array}{ll}\text { Curriculum vitae } & 110\end{array}$

$\begin{array}{ll}\text { Bibliography } & 112\end{array}$ 


\section{Summary}

In this thesis, we present the development and study a numerical model of EUV-induced plasma. Understanding of behavior of low pressure low density plasmas is of industrial relevance, because of their potential use for on-line removal of different forms of contaminations from multilayer mirrors, which will help increase the throughput of EUV lithography.

The model is $2 \mathrm{D}$ axially symmetric particle-in-cell code, hence it allows the full geometry of an axially symmetric chamber to be taken into account. Therefore, a quantitative connection between different experimental data, such as discharge characteristic measurements, and plasma parameters could be established. In order to ensure that the simulations could be relied on for quantitative comparisons, special attention was paid to validating the model. First, the implementation of the model was tested using the accumulated large body of the swarm data to check the values of cross-sections (see chapter 2, section 2.8).

In a second step (see chapter 5), direct simulation of the dynamics of a low-density plasma, ignited by an electron avalanche, in the presence of a diagnostic probe was used as both a validation step and as a first direct comparison between experiment and model. In this study, the plasma, the probe response to the plasma, and the probe's influence on the plasma were all included in the model. Besides validation, the simulated plasma parameters were compared with that estimated from the probe $I V$ curves using a variety of techniques. It was shown that different probe analysis techniques lead to significant under and over estimates of plasma densities From these results, we suggested a useful criterion for estimating the error margin in experiments.

The introduction of EUV radiation into the experimental chamber adds a new layer of unknown and/or poorly known parameters, such as the power spectral density and intensity. These new unknowns were treated, within limits, as free parameters that were varied to obtain close agreement between the numerical model and experimentally measured parameters. This approach was only possible because of the extensive testing and validation of the model, which allowed us to freeze the model state, and concentrate on the effects of added the parameters and new EUV plasma dynamics.

The influence of different parameters on the ignition and dynamics of EUV induced plasmas was studied (see chapter 6). It was found that even low (i.e. 1\%) transmission in the spectrum purity filters in vacuum-ultraviolet wavelength range can have a significant role for EUV-induced plasma formation.

Finally, an experiment with carbon etching, due to EUV induced plasma was considered (see 
chapter 7). It was found that the predicted yield of carbon removal under EUV radiation in a hydrogen atmosphere corresponds well to etching experiments without EUV. This suggests that the underlying physical mechanism is the same in both cases, and that direct EUV processes play an insignificant role in carbon etching.

The developed model is applicable to pulsed low pressure low density EUV generated plasmas. It has proven to be a convenient instrument to help experimentalists understand EUV plasma dynamics. Although the model can be extended in various ways, the most promising, from the perspective of understanding EUV plasma, is the extension of the model to a hybrid plasma model (see Chapter 8). 


\section{Samenvatting}

In dit proefschrift wordt de ontwikkeling gepresenteerd van een numeriek model van Extreem Ultraviolet-geïnduceerde plasma's. Het begrijpen van het gedrag van deze lage druk, lage dichtheid plasma's heeft een belangrijke industriële relevantie vanwege het potentiele gebruik daarvan voor het online verwijderen van verschillende soorten verontreinigingen van multilaagspiegels. Dit is relevant voor de productiviteit van chip-fabricage middels EUV-fotolithografie.

Het onderwerp van het proefschrift betreft een 2D, axiaal-symmetrisch particle-in-cell model, waardoor het mogelijk is om rekening te houden met de volledige geometrie van een axiaal symmetrische kamer. Daarmee kan een kwantitatief verband worden vastgesteld tussen verschillende experimentele data, zoals metingen van karakteristieken van ontlading, en parameters van het plasma. Om er voor te zorgen dat, ook kwantitatief, volledig op de simulatie kan worden vertrouwd, werd bijzondere aandacht geschonken aan de validatie van het model. Allereerst werd de implementatie van het model getest met het gebruik van de geaccumuleerde swarm data om doorsneewaarden te controleren.

Daarnaast werd in een validatiefase directe simulatie van de dynamiek van een lagedichtheidplasma toegepast, welke geïnitieerd is door een elektron-avalanche in aanwezigheid van een diagnostische sonde, en een directe vergelijking tussen experiment en model. In dit onderzoek werden de plasmacondities en de invloed van de sonde op het plasma opgenomen in het model. Behalve validatie, werden ook de gesimuleerde plasmaparameters vergeleken met de parameters die werden geschat op basis van de sondegegevens met gebruik van verschillende technieken. Het kon worden aangetoond dat verschillende technieken van sonde-analyse leiden tot significante over- en/of onderschattingen van de plasmadichtheden. Uit deze resultaten is een effectief criterium voorgesteld voor het schatten van de foutmarge in experimenten.

De introductie van EUV straling in de experimentele kamer brengt een nieuwe serie van onbekende en/of slechts gedeeltelijk bekende parameters met zich mee zoals de spectrale vermogensdichtheid en intensiteit. Deze parameters werden verwerkt als vrije parameters om vervolgens een goede overeenkomst te bereiken tussen het numerieke model en de experimenteel gemeten parameters. Deze aanpak was alleen mogelijk door het uitgebreid testen en valideren van het model, en gaf de mogelijkheid om bij een vast model de effecten van de toegevoegde parameters en de nieuwe dynamiek van het EUV plasma te onderzoeken. De invloed van de verschillende parameters op het initiëren en de dynamiek van het plasma werden ook onderzocht. Het is gebleken dat zelfs een geringe deel-transmissie van toegepaste spectrale filters in het vacuüm-ultraviolet golflengtegebied een belangrijke rol kan spelen bij de vorming van 
EUV-geïnduceerde plasma's.

Ten slotte werd een experiment met het etsen van koolstof door EUV-geïnduceerde plasma's onderzocht. Het bleek dat de verwijdering, ofwel het etsen van koolstof onder EUV belichting in een waterstof-atmosfeer sterk overeenkomt met ets-experimenten zonder EUV. Dit wijst erop dat het onderliggende fysische mechanisme hetzelfde is en dat directe EUV processen een onbelangrijke rol spelen bij het etsen van koolstof.

Het ontwikkelde model is toepasbaar bij gepulseerde, lage druk, lage dichtheid EUV gegenereerde plasma's. Het is een krachtig instrument gebleken dat onderzoekers kan helpen om de dynamiek van EUV plasma's te begrijpen. Hoewel het model op verschillende manieren kan worden uitgebreid, is de meest veelbelovende uitbreiding die tot een hybride plasma model, d.w.z een combinatie van een fluïde plasma model voor het basis plasma met goed gedefiniëerde electronentemperatuur, en een particle-in-cell kinetische beschrijving voor de snelle plasmadeeltjes. Zo'n model zou uiterst relevant zijn voor de condities in EUV lithografische apparatuur. 


\section{Краткое содержание}

Данная работа посвящена численному моделированию динамики плазмы в водороде, индуцированной мягким рентгеновским излучением. Такую плазму возможно использовать для очистки (и поддержания чистоты) поверхности оптических элементов используемых в установках проекционной литографии в диапазоне мягкого рентгена, что обуславливает актуальность данной задачи.

Для моделирования плазмы использовался метод частиц в ячейках в двумерной, цилиндрически симметричной постановке. Такой подход позволил получить количественные оценки для различных величин, измеряемых в эксперименте(например, ток, прошедший через электрод), и соотнести их с параметрами плазмы, полученными в результате моделирования. Для проверки точности модели были проведены тестовые расчеты, в которых проверяли, воспроизводит ли модель результаты экспериментов с дрейфовыми трубами (см. главу 2, раздел 2.8).

Следующим шагом был проведен расчет зондовой характеристики, измеренной в установке, в которой водородная плазма образовывалась в результате развития электронной лавины (см. главу 5). Конфигурация эксперимента была осе-симметричной, что позволило самосогласованно учесть влияние цилиндрического зонда на формирование плазмы. Результаты расчетов и измерений тока на зонд совпали с достаточной точностью, однако параметры плазмы полученные в расчете существенно разошлись с таковыми из анализа зондовой характеристики с помощью стандартных зондовых теорий. Эти различия указывают на то, что стандартные методы анализа зондовых характеристик в случае импульсных плазм должны применяться с осторожностью, и разница между предсказаниями разных моделей отклика зонда может трактоваться как оценка погрешности определения характеристик плазмы.

Успешное моделирование эксперимента с электронной лавиной позволило перейти к более сложному случаю, и рассмотреть формирование плазмы в газе из-за поглощения мягкого рентгеновского излучения. Введение излучения в модель привело к появлению большого количества плохо определенных параметров, таких как интенсивность излучения, временная, пространственная и спектральная форма импульса излучения и т.д. Ввиду невозможности измерения всех необходимых характеристик импульса излучения одновременно с достаточной точностью, эти характеристики рассматривались как свободные переменные с диапазоном изменения определяемыми экспериментальной точностью. 
Влияние различных параметров импульса излучения на характеристики получаемой плазмы было рассмотрено в главе 6). Было обнаружено, что в рассматриваемых условиях даже малое ( 1\%) пропускание используемых спектральных фильтров в области вакуумного ультрафиолета оказывает существенное влияние на формирование плазмы.

В заключении, в главе 7 были проведены расчеты потоков ионов и атомов водорода из плазмы на образец, покрытый углеродом. В этом эксперименте наблюдалась очистка углерода, при этом простые оценки эффективности очистки давали многократно завышенные значения. Однако, оценки потоков, полученные с помощью разработанной модели показали, что эффективность очистки углерода согласуется с ранее измеренными значениями для водородной плазмы низкого давления. Что указывает, на сходство основных механизмов очистки и отсутствие существенного влияния излучения на процесс очистки углерода.

Таким образом, разработанная модель может быть применена к различным задачам связанным с описанием динамики плазмы, индуцированной мягким рентгеновским излучением в водороде низкого давления. Модель является удобным численным инструментом для анализа результатов экспериментов с импульсной плазмой. Перспективным развитием модели может быть обобщение до гибридной плазменной модели (см. главу 8 ). 


\section{Chapter 1}

\section{Introduction}

The general direction of the study presented in this thesis is motivated by the industrial need to describe the ignition and dynamics of low density, low pressure hydrogen plasmas, excited by extreme ultraviolet radiation (EUV), as discussed in Section. 1.1.

These plasmas have many similar properties with low pressure $(1-100 \mathrm{~Pa})$, low density $\left(n_{e} \sim\right.$ $10^{8}-10^{10} 1 / \mathrm{cm}^{3}$ ) pulsed plasmas that are commonly found in many laboratory experiments. These plasmas can exhibit complicated behavior because they are often operated in the nonlocal and non-stationary regime. Therefore, the study of these plasmas has also an academic relevance.

The main part of the study in this thesis is the development of a model (see Section. 2) and application of this model to a selected set of experiments, as discussed in Section. 4.

\subsection{EUV lithography, mirrors and pulsed plasmas}

In the semiconductor industry, photolithography is used to create patterns on silicon wafers, which is an important step in microchip production. Due to diffraction, the minimum size of the characteristic pattern dimensions depends on the wavelength used. Currently, an eximer laser $(\lambda \sim 193 \mathrm{~nm})$ is used as a radiation source, however, the printed feature sizes are significantly smaller than the laser wavelength. The technical measures that are used to circumvent the diffraction-limit are very complicated (e.g. off-axis irradiation, multipole irradiation, multiple patterning, non-linear resists, immersion). A review can be found in a number of textbooks (e.g. [1], [2]).

Since the transition to smaller feature sizes, while keeping the illuminating wavelength the same, is increasingly complicated, one of the possible solutions is to use shorter wavelengths ${ }^{1}$. However, this comes with a set of new problems, since the usual transmission optics cannot be used. Moreover, the shift to reflective optics in turn requires to decrease the wavelength even more $^{2}$, hence the wavelength range for next generation of optical lithography is in the $10-20$ $\mathrm{nm}$ (see introduction in [5]).

The current industry road-map is to shift to $13.5 \mathrm{~nm}$ radiation, i.e. to EUV wavelengths. In order to provide the required imaging performance, interference-based multilayer mirrors (MLM) are used as optical elements. As suggested by the name, MLM optics are based on the constructive interference from multiple layers. However, MLM optics are subject to contamination during exposure to the energetic EUV radiation, due to oxidation and carbon growth.

${ }^{1}$ Examples of the other possible solutions are direct nanoimprint lithography[3], maskless electron-beam lithography[4], etc.

${ }^{2}$ The shift to reflective optics leads to a decrease of the numerical aperture, compared to transmissive optics, this significantly affects the resolution of the imaging system. Therefore, to improve the resolution, the illuminating wavelength must decrease even more. 
Therefore, the study and elaboration of ways to maintain the MLM's reflectivity is interesting and important. Several approaches to increase the MLM's lifetime have been proposed. Most of them involve methods of on-line surface cleaning using active agents, such as hydrogen, or oxygen atoms.

One of the most promising ideas for non-destructive MLM surface cleaning is to use the plasma, produced in the residual gas by the EUV photons and the secondary electrons emitted from the mirror surface, as a cleaning agent. In order to ensure sufficient particle flux to the surface, an additional bias can be applied to the MLM. In this case, the fluxes and energies of charged particles (i.e. ions and electrons) to the MLM surface can be controlled over a large range.

Although experiments have shown that on-line cleaning can be achieved under certain conditions, it has proven difficult to develop an understanding of the processes involved due to two main causes: the characteristics of the EUV-induced plasma are poorly known, and the plasmasurface interaction has many contributing factors [6, 7, 8, 9, 10, 11]. Therefore, an accurate simulation and analysis of such an environment requires both: (a) an estimate of the fluxes from the plasma to the MLM surface and (b) the correct boundary conditions provided by the knowledge of the plasma induced chemistry on the surface. These conditions are addressed in the next section.

\subsection{Industry-related issue and simulations}

To begin with, let us narrow down the parameter space, since a large portion of the parameters can be estimated from the published literature $[12,5,13,14]$. In EUVL, the EUV radiation will be produced by a tin plasma based source $[15,16,17]$ (e.g. a discharge produced plasma (DPP) or laser produced plasma (LPP)).

Despite the fact that EUV sources may provide comparable time averaged EUV output, their characteristics differ significantly in their pulse repetition rate, spectral content, and spatial and temporal coherence, e.g., DPP based EUV source has a repetition rate in the range of $1 \mathrm{kHz}$.. $10 \mathrm{kHz}$, LPP based source has a repetition rate in the range of $10 \mathrm{kHz} . .100 \mathrm{kHz}$.

For a plasma-based source, the EUV pulse duration (approximately $100 \mathrm{~ns}$ ) is much shorter than the time between two consecutive pulses and much longer than the characteristic electron times (e.g. inverse plasma frequency, etc.) in both the solid and the plasma. However, the decay rate of the plasma after the EUV pulse depends on the geometry and volume of the containing vessel. Therefore, considering that, for the commercial use-case, the vacuum chamber is rather large, we limit our models to a single pulse, igniting a plasma in a cold neutral gas.

Next, we can estimate the relevant EUV energy fluence per pulse. The industry roadmap mentions a broad range of the energies from $100 \mathrm{~W}$ to $1 \mathrm{~kW}$ of EUV power ${ }^{3}$ at the intermediate focus. ${ }^{4}$ However, to estimate the energy fluence per pulse, we need the optics' dimensions. Given the two orders of magnitude arising from the possible range of source repetition rates, it is safe to estimate the diameter of the optics to be about $10 \mathrm{~cm}$. Note that dimensions of $1 \mathrm{~cm}$ are

\footnotetext{
${ }^{3}$ The EUV power relevant to EUVL is the power emitted by the source in a $2 \%$ bandwidth around $13.5 \mathrm{~nm}$ [15].

${ }^{4}$ Intermediate focus is the point in the optical path, where EUV radiation exits the source chamber and enters the chamber with optics.
} 
unlikely due to diffraction, while on the other hand, MLM optics with a $100 \mathrm{~cm}$ characteristic size are only subject to very low EUV intensities and have less relevance. From these estimates, we consider that the relevant energy fluence range for $250 \mathrm{~W}$ sources is $10^{-2} . .1 \mathrm{~mJ} /($ pulse $\times$ $\left.\mathrm{cm}^{2}\right)^{5}$.

The next step is to estimate the plasma density. Due to the fact that EUV radiation is very effectively absorbed by all materials, the background gas environment is expected to have a low pressure The pressure range, based on known EUVL conditions, ranges between $1-100 \mathrm{~Pa}$ hydrogen [18]. ${ }^{6}$ For these conditions (i.e. $10^{-2} \mathrm{~mJ} /\left(\right.$ pulse $\left.\times \mathrm{cm}^{2}\right)-1 \mathrm{~mJ} /\left(\right.$ pulse $\left.\times \mathrm{cm}^{2}\right)$ ), the plasma density can be estimated as $10^{7} . .10^{11} 1 / \mathrm{cm}^{3}$, which is similar to the density of the plasma found in glow discharges. However, important differences in the plasma dynamics originate from the pulsed radiation induced nature of the plasma.

A glow discharge plasma is quasi-stationary and the energy distribution function (EDF) for electrons is close to Maxwellian. In contrast, as photoionization makes a significant contribution to the EUV induced plasma formation, the electron EDF deviates significantly from a Maxwell distribution function during the EUV pulse and for some time after it. In spite of the presence of some fast electrons that might remain some time after the EUV pulse, the total energy decreases monotonically, therefore, the plasma starts to decay due to ambipolar diffusion.

Therefore, because of the non-stationary and non-Maxwellian nature of EUV induced plasmas, we need to solve the equations for the electron distribution function, $f_{e}$, without any assumption about its shape in advance. This rules out a significant class of simulation methods, because the fluid approximation cannot be applied. Here, by fluid approximation, we mean a class of methods that relies on continuity equations for moments of the distribution function, e.g. electron density $n_{e}=\int f_{e}(t, \vec{x}, \vec{v}) d \vec{v}$, flow $\left\langle n_{e} \vec{v}_{e}\right\rangle=\int \vec{v} f_{e}(t, \vec{x}, \vec{v}) d \vec{v}$, etc. However, $f_{e}$ is unknown, thus, a kinetic approach, which doesn't rely on an explicit form for $f_{e}$, should be used.

Unfortunately, kinetic methods are much more computationally intensive compared to fluid based methods, because these methods solve equations for the distribution function. Hence, let us estimate the problem size in terms of characteristic plasma length and time scales: the Debye radius, and plasma frequency. Even though an EUV induced plasma has a Debye radius and plasma frequency that evolves with time, it is reasonable to assume a single value for each, since the low energy part (i.e. the energy range up to several times the temperature) of the Maxwell energy distribution forms very fast due to inelastic collisions with the background gas and interactions via the electric field.

For the purpose of estimating the Debye radius, we assume $T_{e} \sim 0.5 \mathrm{eV}$, since this is a typical for electrons in low density hydrogen plasmas. The Debye radius for a plasma with $T_{e} \sim 0.5 \mathrm{eV}$ and $N_{e} \sim 10^{9} 1 / \mathrm{cm}^{3}$ is $R_{D} \sim 0.015 \mathrm{~cm}$, i.e. the typical length scale of a laboratory experiment is $10 \mathrm{~cm} \sim 600 \times R_{D}$. A reasonable time scale is $1 \mu \mathrm{s}$, since the duration of an EUV pulse from a plasma based source is about $0.1 \mu \mathrm{s}$. Using the parameters above, the plasma frequency is about

${ }^{5}$ The energy fluence per pulse is (EUV power)/(rep. rate. $\times$ surface area). EUV source repetition rates vary greatly, the common range being $1 . .4 \mathrm{kHz}$ for DPP and $10 . .50 \mathrm{kHz}$ for LPP [15, 17]. For this estimate we choose a repetition rate range of $4 . .50 \mathrm{kHz}$ and round the results to lower and higher values respectively. The out of band EUV radiation is not taken into account in this estimate, because, for LPP based source, this contribution increase radiation fluence by a factor of two.

${ }^{6}$ The pressure range can be estimated by considering the EUV absorbtion in the volume. For several (e.g. 6 - 8) mirrors with characteristic size about $10 \mathrm{~cm}$ the optical path should be about $100 \mathrm{~cm}$. Hence, $\sim 13 \%$ of EUV radiation is absorbed by gas for $100 \mathrm{~Pa} \mathrm{H}_{2}$ background pressure. Therefore, higher pressure of background gas seems to be impractical. 
$2 \mathrm{GHz}: 1 \mu \mathrm{s} \sim 2000 / \omega_{p l}$.

Given these estimations, the natural choice is to apply a method from the Particle-in-Cell (PIC) family This kind of method requires that the Debye radius and plasma frequency should be resolved, fortunately, under the conditions described above, this seems to be a feasible task. ${ }^{7}$

The PIC method also has the advantage of being able to describe the plasma sheath in a selfconsistent manner, i.e. no special plasma sheath models are required, as is often the case for hybrid and fluid models. Thus, we can straightforwardly obtain the energy resolved ion fluxes from the EUV induced plasma to the surface of the MLM mirror through the PIC simulations, which is the goal of the present work.

\section{3 "Why a new code"}

There is no standard program for plasma physics related problems, instead, there are many codes, designed by different laboratories for their specific purposes. Because of the extremely large number of codes, it is impossible to prove that there is no pre-existing code that could be used to solve the problems considered in this thesis.

Most probably there are some codes, which, if combined and provided with the correct input, could be used to accomplish this task. But, it would require (a) finding such codes, (b) adding (or fine-tuning) code that is specific to the problems that we consider, and (c) testing its applicability, since each code is tailored towards a specific set of problems. In fact, overcoming these problems is not simpler than writing a new code that is tailored towards our specific problems from the beginning.

More specifically, we need to describe, in 2D, the fast evolution of a low pressure, low density hydrogen plasma, and compute the ion flux from the plasma to the surface. This, in turn, requires a kinetic description for both ions and electrons, dealing with many reactions between plasma species and the background gas, and an appropriate description of the plasma sheath. This combination is not found in the freely available codes for simulations of low pressure low density plasmas, since these features are not required for common tasks, such as a stationary glow discharge in 1D, complex plasma chemistry for an almost stationary plasma using a fluid description, e.g. for material processing in lithography, and the evolution of a plasma with relatively simple chemistry in 1-3D (e.g. plasma thrusters for space applications), etc.

A few examples of plasma simulation codes, which are available for download include: XOOPIC [19], Magbolts [20], BOLSIG+ [21]. There are many other closed source codes, e.g. Starfish, PLASIMO, VSIM and many others. However, all of these codes would require significant modifications before they could be used to study EUV-induced, low density hydrogen plasmas.

Hence, most likely, at least two codes would need to be merged to obtain the required set of features. In view of the completely different internal structure of the different codes this seems to be a hopeless task. Therefore, we have developed a new code, which is described in next section.

${ }^{7}$ By resolving the Debye radius and plasma frequency, we mean that the grid spacing should be less than $3.4 R_{D}$, and the time step should be less than $2 / \omega_{p l}$. However, a much stricter condition for the time step is used, e.g. $\Delta t<$ $0.1 / \omega_{p l}$. Otherwise, the error in the total energy is often unacceptably high. Hence, to model a region with a size of $600 R_{D} \times 600 R_{D}$ we need approximately $200 \times 200$ grid points, and at least $10^{5}$ super particles. For modern computers this is not a big problem. 
EUV induced plasmas have been considered in 1D with argon as background gas [22], [23]. We did not use this work as a starting point, because we need to deal with hydrogen in $2 \mathrm{D}$. Some parts of the code (e.g. the initial version of the cross-sections set) and design decisions of the model developed in this thesis are based on an unpublished 1D low pressure hydrogen plasma model, for which we acknowledge the contribution of Vladimir Ivanov. 



\section{Chapter 2}

\section{Numerical tool}

\subsection{Plasma equations}

The are many variations in the formulation of particle based methods for simulations of plasma dynamics. For clarity, in this section, we describe the route from the equations of state for a plasma to the numerical scheme used in our code, which follows [24]. A detailed description of plasma physics and numerical methods can be found in appropriate textbooks (e.g., [25]).

The kinetic equations for a plasma (Vlasov - Boltzmann equations) read

$$
\begin{gathered}
\frac{\partial f_{a}}{\partial t}+\vec{v} \vec{\nabla}_{\vec{r}} f_{a}+\frac{\vec{F}_{a}}{m_{a}} \vec{\nabla}_{\vec{v}} f_{a}=S t(f), \\
\vec{F}_{a}=q_{a}\left(\vec{E}+\frac{1}{c} \vec{v}_{a} \times \vec{H}\right)
\end{gathered}
$$

where $f_{a}$ is the distribution function for a species, $a$, with mass $m_{a}$ and charge $q_{a} . S t(f)$ is the term which accounts for the effect of collisions. $S t(f)$ has a complicated form, but, fortunately we do not need to expand it explicitly, because, in our model, we use another approach to introduce collisions (see Section. 2.7). To these equations one should add the Maxwell equations for the field.

$$
\begin{gathered}
\operatorname{div} \vec{E}=4 \pi \rho, \quad \operatorname{rot} \vec{H}=\frac{1}{c} \frac{\partial \vec{E}}{\partial t}+\frac{4 \pi}{c} \vec{j}, \quad \operatorname{rot} \vec{E}=-\frac{1}{c} \frac{\partial \vec{H}}{\partial t}, \quad \operatorname{div} \vec{H}=0 \\
\rho=\sum_{a} q_{a} \int_{-\infty}^{\infty} f_{a} d \vec{v}, \quad \vec{j}=\sum_{a} q_{a} \int_{-\infty}^{\infty} f_{a} \vec{v} d \vec{v}
\end{gathered}
$$

where the symbols have their usual definitions. In the considered case, there is no strong external magnetic field, and the magnetic field generated by the plasma is negligible (i.e. $|\vec{H}| \ll|\vec{E}|$ ). Thus, is it possible to use the electrostatic approximation, i.e.

$$
\begin{gathered}
\frac{\partial f_{a}}{\partial t}+\vec{v} \vec{\nabla}_{\vec{r}} f_{a}+\frac{q_{a} \vec{E}_{a}}{m_{a}} \vec{\nabla}_{\vec{v}} f_{a}=S t(f), \\
\vec{E}=-\vec{\nabla} \varphi, \quad \Delta \varphi=-4 \pi \rho
\end{gathered}
$$

It is not possible to solve these nonlinear equations analytically in any real-life related case. For a numerical solution, some approximation for the distribution function $f_{a}$ should be made, e.g. both fluid and kinetic descriptions use an approximation for the distribution function, but the PIC method allows a broader class of possible distribution functions to be used compared to fluid 
methods.

For the fluid description, the distribution function is multiplied with powers of the velocity and integrated over velocity space, yielding moments of the distribution function. For example: the electron density is $n_{e}=\int f_{e}(t, \vec{x} d \vec{v}, \vec{v})$, electron flux (i.e. current) $\left\langle n_{e} \overrightarrow{v_{e}}\right\rangle=\int \vec{v} f_{e}(t, \vec{x}, \vec{v}) d \vec{v}$, electron energy density $\left\langle n_{e} \frac{m_{e} v_{e}^{2}}{2}\right\rangle=\frac{1}{2} m_{e} \int(\vec{v} \cdot \vec{v}) f_{e}(t, \vec{x}, \vec{v}) d \vec{v}$, etc. Then, continuity equations can be obtained for the electron density, current density etc. A fluid model needs to be coupled to an additional model to describe the plasma sheath, because the plasma sheath region generally cannot be described in the continuous matter approximation.

As was mentioned previously, the fluid approach requires some a priori assumptions about the shape of the distribution function to close the system of equations, since the lower moments of the distribution function depend on higher moments, i.e. electron density on the electrons flux, electron flux on energy density, etc. Unfortunately, in the present case it is hard to make a good approximation for the distribution function in advance.

For the Particle-in-Cell method, the distribution function is approximated as follows

$$
f_{a}(t, \vec{x}, \vec{v})=\sum_{i_{a}} w_{i_{a}} \delta\left(\vec{v}-\vec{v}_{i_{a}}\right) S\left(\vec{x}-\vec{x}_{i_{a}}\right), \quad \int_{V} S(\vec{x}) d \vec{x}=1
$$

Here $w_{i_{a}}>0, \vec{x}_{i_{a}}$ and $\vec{v}_{i_{a}}$ are the parameters that correspond to the weight, coordinate, and velocity of the $i$ th super particle of species $a$, i.e. a super particle is a term in sum in (2.7), because it represents an object with a certain coordinate and velocity. The function $S$, which is referred to as a shape function is non-negative (i.e. for any input, $S(\vec{x}) \geq 0$ ).

The weight parameter corresponds to the number of particles that are represented by a super particle. To illustrate this statement, let us integrate the distribution function over phase space.

$$
N^{a}(t)=\int_{V} d \vec{x} \int_{-\infty}^{\infty} d \vec{v} f_{a}(t, \vec{x}, \vec{v})=\sum_{i_{a}} w_{i_{a}}
$$

Hence, $w_{i_{a}}$ corresponds to a number of particles, because the distribution function $f_{a}$ is normalized to the full number of particles, $N^{a}$, for species $a$ in the simulated volume $V$.

It is possible to use any shape function (2.9). However, the delta function is more convenient when one needs to deal with an irregularly spaced grid, otherwise, the right hand side of equations of motion, which we obtain at the end of this section (i.e. equations (2.14) and (2.15)), have a more complicated form. Therefore, we use the following approximation for the distribution function, which have delta functions for velocity and position.

$$
f_{a}(t, \vec{x}, \vec{v})=\sum_{i_{a}} w_{i_{a}} \delta\left(\vec{v}-\vec{v}_{i_{a}}\right) \delta\left(\vec{x}-\vec{x}_{i_{a}}\right)
$$

Despite the fact that it is possible to obtain equations for $\vec{x}_{i_{a}}$ and $\vec{v}_{i_{a}}$ by substituting (2.9) to (2.5), it is more convenient to use an effective Lagrangian [26, 27].

The numerical versions of Poisson's equation and the equations of motion for particles (i.e. (2.6) and (2.14)) should be consistent with each other, i.e. there should be a certain relationship between the particle's shape function, the order of the numerical representation of the force 
acting on the particle, and the approximation order of the discretization of Poisson's equation.

The common approach is to use certain heuristic rules to derive a consistent numerical scheme. Unfortunately, it is hard to apply these rules for any case other than a uniform rectangular grid. On the other hand, the derivation of equations of motion via the effective Lagrangian is free from the above mentioned issues.

The effective Lagrangian that leads to (2.5) without a collision term can be written as follows.

$$
L=\sum_{a} \int d \vec{x} d \vec{v} f_{a}(t, \vec{x}, \vec{v})\left(\frac{m_{a} v^{2}}{2}-q_{a} \varphi(\vec{x})\right)+\frac{1}{8 \pi} \int d \vec{x}(\vec{\nabla} \varphi(\vec{x}))^{2}
$$

The index $a$ denotes species, e.g. electrons or hydrogen ions, etc.

Before we proceed further, let us introduce a spatial grid for the potential. We use the finite element method to approximate the potential on the grid.

$$
\varphi(t, \vec{x})=\sum_{j} \alpha_{j}(t) \xi_{j}(\vec{x})
$$

Here the index $j$ runs over all grid points, the functions $\xi_{j}(\vec{x})$ form a set of basis functions, weighted by time-varying coefficients, $\alpha_{j}$, which, together, represent the electric field potential $\varphi(t, \vec{x})$ with sufficient accuracy. In our model, we choose simple basis functions as discussed later (2.16).

Substituting (2.9) and (2.11) into the first term of (2.10) and integrating yields the formula ${ }^{1}$

$$
L=\sum_{a} \sum_{i_{a}} w_{i_{a}}\left(\frac{m_{a} v_{i_{a}}^{2}}{2}-q_{a} \sum_{j} \alpha_{j}(t) \xi_{j}\left(\vec{x}_{i_{a}}\right)\right)+\frac{1}{8 \pi} \int d \vec{x}(\vec{\nabla} \varphi(\vec{x}))^{2}
$$

The geometry we are interested in possesses cylindrical symmetry, therefore, it is convenient to change to cylindrical coordinates for the last term of (2.12) and integrate over the polar angle before we apply the Euler-Lagrange equations to obtain the equations of motion. We do not change coordinates in the first term of (2.12) since it is convenient to trace super particles in 3D, but solve Poisson's equation in 2D. Then we obtain

$$
\begin{aligned}
L=\sum_{a} \sum_{i_{a}} w_{i_{a}}\left(\frac{m_{a} v_{i_{a}}^{2}}{2}-q_{a} \sum_{j} \alpha_{j}(t) \xi_{j}\left(\vec{x}_{i_{a}}\right)\right)+\frac{1}{4} \int r d r d z\left(\vec{\nabla}_{r, z} \varphi(r, z)\right)^{2}= \\
=\sum_{a} \sum_{i_{a}} w_{i_{a}}\left(\frac{m_{a} v_{i_{a}}^{2}}{2}-q_{a} \sum_{j} \alpha_{j}(t) \xi_{j}\left(\vec{x}_{i_{a}}\right)\right)+ \\
+\frac{1}{4} \sum_{j} \sum_{k} \alpha_{j}(t) \alpha_{k}(t) \int r d r d z\left(\vec{\nabla}_{r, z} \xi_{j} \cdot \vec{\nabla}_{r, z} \xi_{k}\right)
\end{aligned}
$$

Finally, with the help of the Euler-Lagrange equations, the equations of motion are obtained for

${ }^{1}$ Here, we take advantage of our simple form for $f_{a}$, which allows the integral of the first term of the Lagrangian to be performed analytically. 
the dynamic variables (e.g. $\vec{x}_{i_{a}}, \vec{v}_{i_{a}}=\dot{\vec{x}}_{i_{a}}$ and $\alpha_{j}$ )

$$
\begin{gathered}
\ddot{\vec{x}}_{i_{a}}=-\frac{q_{a}}{m_{a}} \vec{\nabla} \varphi=-\frac{q_{a}}{m_{a}} \sum_{j} \alpha_{j} \vec{\nabla} \xi\left(x_{i_{a}}\right) \\
\sum_{j} \alpha_{j} \int r d r d z\left(\vec{\nabla}_{r, z} \xi_{j} \cdot \vec{\nabla}_{r, z} \xi_{k}\right)=2 \sum_{a} q_{a} \sum_{i_{a}} w_{i_{a}} \xi_{k}\left(x_{i_{a}}\right)
\end{gathered}
$$

As we obtained equations (2.14) and (2.15) via the Lagrangian approach, the conserved quantity is energy in the limit of infinitely small time steps. Unfortunately, the momentum is not conserved because the grid breaks the symmetry to infinitesimal shifts, thus Noether's theorem cannot be applied.

Although the failure of momentum conservation is a problem, it is a phase error on the level of one super particle oscillating at the plasma frequency in an effective potential. For the case where oscillations of an external field are the dominate source of energy for the system and collisions between charged species and background gas are negligible, phase errors become very significant. In such cases, the phase error changes the amount of energy flowing into the system.

However, in our case, the relevant conditions are that collisions between plasma species and the background gas are significant. These collisions randomise the oscillation phases of the super particles. The overall momentum is not conserved, since, in the model, we discard slow neutral species, which are produced by collisions. Therefore, the numerical scheme should only be accurate enough to not produce an excessive phase error between two collisions. Finally, the main energy source for the EUV-induced plasma is provided by the EUV radiation itself and a DC external bias (if present). These factors are insensitive to the phase of the plasma oscillations. Therefore, for our task, conservation of energy by the numerical scheme is more important than conservation of momentum.

\subsubsection{Energy conserving scheme vs momentum conserving scheme}

The difference between the energy conserving scheme and the momentum conserving scheme, on the level of equations, comes from the difference between the right hand side of (2.14) for each case. For the energy conserving scheme, in the right hand side of equation (2.14), the local field acts on the particle, i.e. no information from the nearby cells is used. For the momentum conserving scheme, a slightly different procedure is used [28]. Firstly, the field gradients are computed for the grid points, secondly these gradients are interpolated to the positions of the super particles. This procedure is not trivial, because the right hand side does not coincide with the local potential gradient. The approach used for the momentum conserved scheme can be viewed as field smoothing in terms of the energy conserving scheme, i.e. local field gradients are used in the energy conserving scheme, while averaged local field gradients are used in the momentum conserving scheme.

For example let us consider a 1D situation with a field distribution as in Fig. 2.1. Note that the electric field at the grid points (i.e. $\vec{E}$ ) is zero if it is computed as the spatial derivative of the potential by the symmetric scheme (i.e. $E_{j}=-\frac{\varphi_{j+1}-\varphi_{j-1}}{2 h}$ ).

Thus, if we simulate the super particle movement in this potential with the momentum con- 

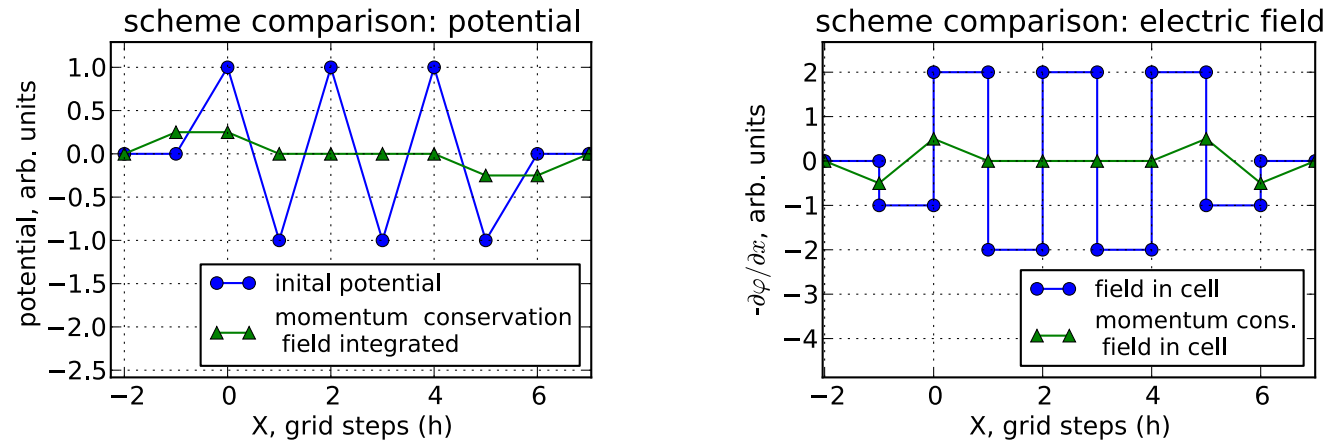

Figure 2.1: In the left plot, the initial model potential with a high frequency oscillation is compared to the effective potential that the particle sees in the momentum conserving case. The effective potential is obtained by integrating the field strength for the momentum conserved scheme from the right plot. The high frequency oscillation of potential is effectively removed in the momentum conservation scheme approach, as compared to energy conservation scheme approach.

serving scheme, under the assumption that the kinetic energy of the particle is much larger than the variations of the potential, we would obtain a super particle that goes straight, neither gaining nor loosing energy. Over longer distance scales, this is correct, but it is not correct for a single super particle in detail, since the local potential variations are not taken into account However, if the potential variations are comparable or larger than the particle kinetic energy this approach would yield an incorrect solution.

If we simulate super particle movement in this potential with the energy conserving scheme, then there would be a non-zero force acting on the particle, because the local field gradient is computed to be $E_{l o c}=-\frac{\varphi_{j+1}-\varphi_{j}}{h}$. If we apply Euler's scheme for the time discretization of (2.14), energy is not conserved for particles that cross grid points during a time step, because of the significant potential gradient changes at the grid points (see Fig. 2.1 and Fig. 2.2). For the full simulation (i.e. with equations (2.15) included) this would result in a significant artificial increase in the temperature unless the time step is very small, i.e. $\Delta t \ll \frac{h}{v}$ for all particles.

Since our task requires dealing with plasma sheaths, and space charge generated potential barriers, the super particle kinetic energy is comparable to the potential variations. This poses a problem for the momentum conserving scheme, because the particle would see an incorrect potential near a sharp potential barrier see Fig. 2.2. That would lead to a situation where the super particle would not see the full potential barrier, which would lead to significant errors in energy conservation.

Unfortunately, the same situation is also troublesome for the energy conserving scheme, since we need to consider a broad energy spectrum for the electrons, because there can be very hot and very cold electrons present at the same time in the simulation domain (see Section 2.6). While, for the majority of the particles, the time scale is the plasma frequency, the overall time step is governed by the fast minority, in combination with the size of the smallest cells (which corresponds to plasma sheath or space charge potential barrier). This significantly slows down the simulation process. 

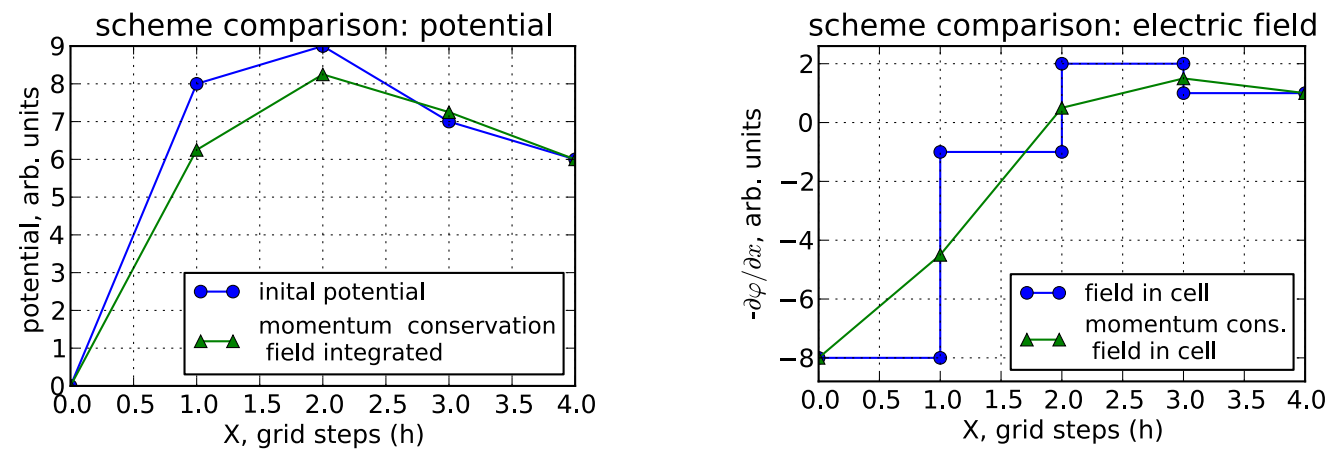

Figure 2.2: Comparison of the initial potential distribution with the effective potential for a momentum conserving scheme. Due to the smoothing effect, the maximum value of the effective potential is lower than for the initial potential. Thus, the particle, which starts from $X=0$ can go under the potential barrier in the momentum conserving scheme, but not in the energy conserving scheme. For example, a particle, which starts from $X=0$ with energy 8.5 arb. units, overcomes the barrier in the case of momentum conserving scheme. To prevent this situation one would need to increase the grid resolution.

However, under the relevant conditions, the plasma sheath and space charge potential barrier are an almost constant background for the high energy electron contribution to the EDF, since many slow particles participate in the potential build-up.

Therefore, it is possible to use a sub stepping scheme, i.e. at each time step we determine whether a particle crosses the cell boundary or not. If the cell boundary is not crossed, then the usual time step is used. In the case where the particle would cross the boundary, we find a series of sub steps that sum to the longer time step used for the field calculation, and, at the end of one sub step, the particle is located on the cell boundary. This approach reduces the computational cost, since the additional time steps are only applied to particles that require it, and allows the simulations to resolve both short and long time-scale behaviour.

\subsection{Program structure}

Our implementation of a Particle-In-Cell (PIC) model with Monte-Carlo (MC) collisions is intended to numerically solve equations (2.14) and (2.15) with an empirical collision term. The flow chart of the main loop is presented in Fig. 2.3.

We use an explicit method to solve the numerical equations derived in section 2.1. Therefore, the main loop consists of a Poisson's equation solver, which is followed by updating the particle's positions and velocities based on the obtained field distribution and current particle's velocities. The new charge distribution is calculated from the updated particle's positions and used as input for the next iteration. At each time step, new particles can be injected into the simulation domain, if required. This loop iterates, advancing time by $d t$ until the required simulation time is reached.

The computation of the charge distribution (i.e. the right-hand side of equation (2.15)) is often called as the charge to grid deposition step. During this step, the charge contained by each super-particle is attributed to grid points using (2.16) and (2.15). It is important to optimise this 


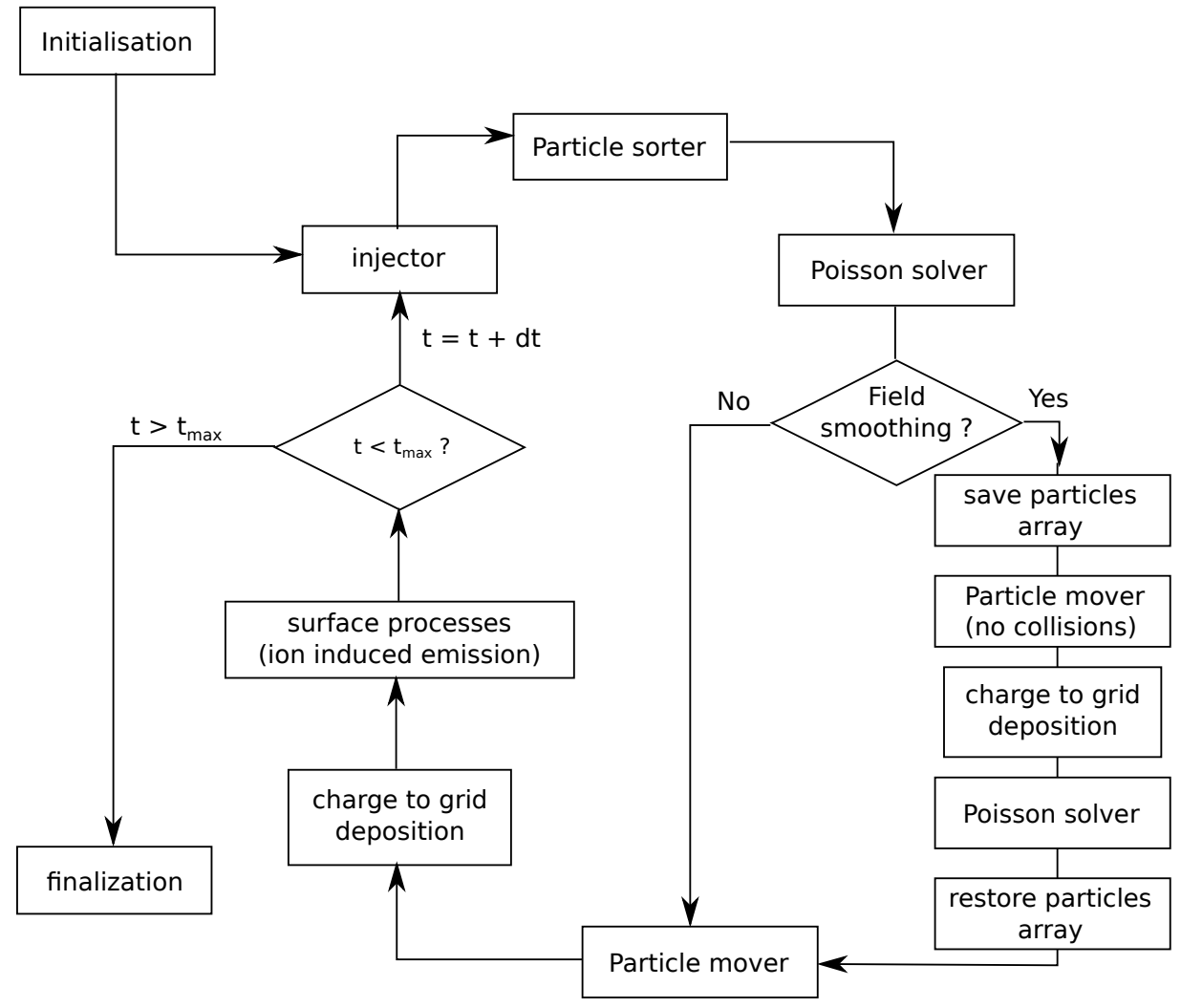

Figure 2.3: Flow chart of a main loop.

part of the main loop, because it consumes a significant amount of computational time.

The optimisation takes the form of memory management. A particle sorter step keeps particles from the same cell nearby in the computer's memory. This technique improves the particlebased code performance because of the more effective use of the memory bandwidth. If no special measures are taken, the relationship between the particle index in the particles array and the cell index is rather random, i.e. each particle will be loaded from the main system memory, rather than from the faster cache memory, since the chances that it is in the cache are negligible. In our implementation, the information about the entrance and exit of particles from a cell (calculated by the particle mover) is used to count the number of particles in each cell in advance. After all the particles are moved, the local particle arrays are merged with the help of a bucket sort by the cell index.

To reduce the numerical noise, an optional sub-loop can be switched on. In this loop, Poisson's equation is solved at intermediate intervals (e.g., $d t / 2$ ). To do this, the particles are temporarily advanced to intermediate locations (without collisions), everything except the updated charge distribution is discarded, and Poisson's equation is used to calculate the field with this updated charge distribution. Then, the super-particles are moved from their old positions in the updated field distribution. 
All the other components of the main loop are described below.

\subsection{Grid}

A rectilinear grid with non-uniform grid spacing is used to resolve the plasma sheath and other spatially sharp features (e.g. a plasma probe).

The nodal decomposition of the potential from (2.11) is based on linear interpolation functions

$$
\begin{aligned}
\xi_{i j}(r, z)=A_{i}(r) \cdot B_{j}(z), \\
A_{i}(r)=\left\{\begin{array}{l}
\frac{r_{i-1}-r}{r_{i-1}-r_{i}}, r \in\left[r_{i-1}, r_{i}\right] \\
\frac{r_{i+1}-r}{r_{i+1}-r_{i}}, r \in\left[r_{i}, r_{i+1}\right]
\end{array} \quad, \quad B_{j}(z)=\left\{\begin{array}{l}
\frac{z_{j-1}-z}{z_{j-1}-z_{j}}, z \in\left[z_{j-1}, z_{j}\right] \\
\frac{z_{j+1}-z}{z_{j+1}-z_{j}}, z \in\left[z_{j}, z_{j+1}\right]
\end{array}\right.\right.
\end{aligned}
$$

Internal electrodes are introduced by fixing the potential of the grid cells that contain the electrode, as shown in the example presented in Fig. 2.4. The electrodes absorb all incoming particles. On ion impact, a secondary electron may be emitted from the electrode surface with a certain probability as discussed in section 2.9.

There is also support for dielectric internal structures, which are needed for simulating the plasma probe with a probe shield. Dielectrics are treated the same way as electrodes in that they absorb incoming particles, but, unlike electrodes, accumulate incoming charge. The potential on the grid nodes that belong to dielectric structures is determined via solution of Poisson's equation.

\subsection{Poisson solver}

To simulate the plasma evolution in time we need to solve Poisson's equation (2.15) at every time step. The efficiency of the Poisson solver significantly influences the code run time, because the number of numerical operations required to solve Poisson's equation is typically comparable to that required to make one step in time for the particles.

There are many methods to solve Poisson-like equations. Let us rewrite (2.15) in matrix form, i.e.

$$
A \vec{\alpha}=\vec{b}
$$

here $A$ is the matrix of coefficients $a_{j k}$ and $\vec{b}$ is a vector of corresponding right hand side terms $b_{k}$

$$
a_{j k}=\int r d r d z\left(\vec{\nabla}_{r, z} \xi_{j} \cdot \vec{\nabla}_{r, z} \xi_{k}\right), \quad b_{k}=2 \sum_{a} q_{a} \sum_{i_{a}} w_{i_{a}} \xi_{k}\left(x_{i_{a}}\right),
$$

and $\vec{\alpha}$ consists of time dependent coefficients from the finite element discretisation of the potential (2.11).

Since the coefficients matrix $A$ only depends on the grid, we can choose a method with a reasonably expensive setup procedure, because we can expect that, for the selected grid, we must solve (2.17) more than $10^{4}$ times. 


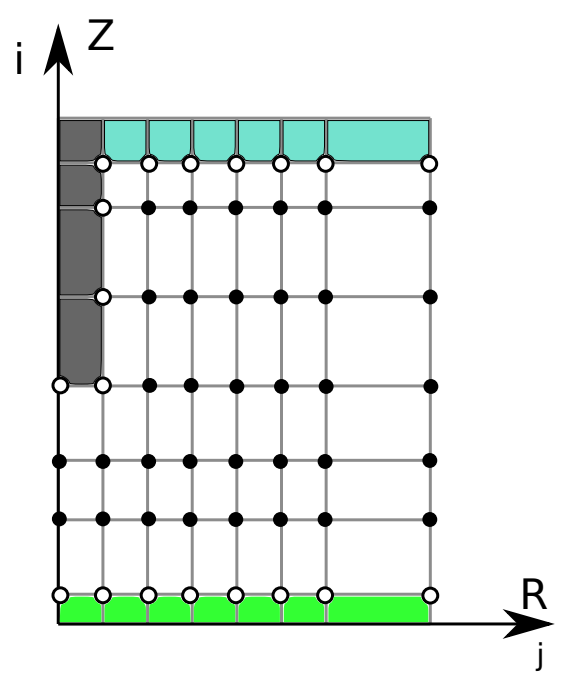

Figure 2.4: Example of a grid with three internal electrodes (colored boxes). Grid nodes marked with open points have the fixed potential of the electrodes. Solid points indicate nodes with a variable potential. Note, that solid points on the boundary represent a free boundary condition for a finite element method. On the right boundary, this condition mimics the situation of infinite free space. The electrodes absorb incoming all incoming particles, and in certain cases, emit secondary electrons (see section 2.9).

As the time step is, at least, restricted to $\Delta t<1 / \omega_{p l}$, the variation of the right-hand side of (2.17) and (2.15) are small between consecutive time steps, i.e. on average, the variations of $x_{i_{a}}$ are small compared to the cell size, therefore, the variations of $\xi_{k}\left(x_{i_{a}}\right)$ are also small. Thus, the solution from the previous time step is a good guess for the solution at the present time step, i.e. the natural choice is an iterative method. Therefore, our choice is a multi-grid method, because it is $\mathrm{O}(\mathrm{N})$, iterative, and benefits from an accurate setup phase, which should be done only once for a particular discretization (2.17) of Poisson's equation.

\subsubsection{Multigrid method}

In this section, we only describe the general ideas of the method and the potential difficulties implementing the method. More details can be found in many good books about multi-grid methods [29, 30, 31, 32].

The multi-grid method is based on several observations. Firstly, equation (2.17) is linear, thus, once we have an approximate solution $\vec{x}^{0}$, we can reformulate the problem for solving (2.17) to solving a correction equation. Let us define the residual, $\vec{r}$, as

$$
\vec{r}=A \vec{x}^{0}-\vec{b}
$$

here, and in the following discussion, $A$ and $\vec{b}$ are the same as in (2.17). Then, the correction equation is

$$
A \vec{\delta}=\vec{r}
$$


Due to the linearity of (2.17) and (2.20), if the correction, $\vec{\delta}$, is found for some guess $\vec{x}^{0}$, then the solution to equation (2.17) is $\vec{x}=\vec{x}^{0}+\vec{\delta}$. Unfortunately, solving equation (2.20) is as difficult as solving (2.17), if both are considered on the same grid.

Secondly, the classic iterative methods (i.e. Gauss-Seidel iterations) for solving (2.17) dump the residual (i.e. $\vec{r}$ ) non-uniformly in the spatial frequency domain. The residuals for the high spatial frequency components reduce very rapidly, requiring only a few iterations. But, the residuals of the low frequency components reduce very slowly. This is a consequence of the local nature of iterative algorithms based on matrix splitting. ${ }^{2}$

Thirdly, the linear system (2.17) represents a discretization of Poison's equation. Because the numerical scheme used for discretization of the initial equation is an approximation, i.e. the accuracy of a numerical solution increases with the increase of the grid resolution, the solution of the same equation on a coarser grid should be reasonably close to the solution on a fine grid, if both grids have comparable resolution. ${ }^{3}$

Therefore, after several iterations of a relaxation algorithm, such as Gauss-Seidel, for equation (2.17), the residual, as defined by (2.19), has only low spatial frequency components (i.e. it is "smooth"). Hence, the residual can be accurately represented on coarser grid. Then, the correction equation (2.20) can be solved on the coarse grid. After the solution for the correction equation is found, it can be interpolated on the fine grid. This procedure allows the low spatial frequency components of the residual to converge quicker, because, on the coarse grid, they correspond to the high frequency components.

These observations can be summarised by a two-stage iterative algorithm. First, the relaxation algorithm is applied to the previous approximation of solution.

$$
\vec{x}_{h}^{i}=S_{h}\left(A_{h}, \vec{b}, \vec{x}_{h}^{i-1}\right)
$$

Here $S_{h}\left(A_{h}, \vec{b}, \vec{x}_{h}^{i-1}\right)$ - the relaxation operator, which is based on an approximate solution of equation $A_{h} \vec{x}_{h}^{i-1}=\vec{b}$ on the fine grid, $A_{h}$ is the coefficients matrix on the fine grid being the same as in (2.17). The main purpose of this step is to significantly decrease the high spatial frequencies components of the error. Fast convergence is not required for this step.

Then, the residual is computed and interpolated on the coarse grid.

$$
\begin{gathered}
\vec{r}_{h}=A_{h} \vec{x}_{h}^{i}-\vec{b} \\
\vec{r}_{2 h}=I^{T} \vec{r}_{h}
\end{gathered}
$$

Here $I^{T}$ is the transpose of interpolation operator from coarse to fine grids. Note, that the values defined on the fine grid have subindex $h$, while the values defined on the coarse grid have subindex $2 h$.

${ }^{2}$ With our definition of support functions there are, at most, nine non-zero coefficients in each row of matrix $A$. Therefore, the number of iterations required for one location in the grid to influence another location is about the distance between the two grid locations (counted in grid points) for the Gauss-Seidel or Jacobi methods. Hence, errors with a large spatial scale are removed last.

${ }^{3}$ Interestingly, if the same reasoning is applied recursively, then it is worth starting with a very coarse grid and gradually increasing the grid resolution. This is the basis of the so-called full multigrid approach, which allows a very good first guess (i.e. $\vec{x}^{0}$ ) to be obtained for the solution of equation (2.17). However, for our task, the best first approximation is the solution of Poisson's equation from the previous time step. 
Hence, after interpolation, we obtain a correction equation on the coarse grid.

$$
A_{2 h} \vec{\delta}_{2 h}=\vec{r}_{2 h}
$$

here $A_{2 h}$ is the coefficients matrix on the coarse grid, constructed from the interpolation operators and $A_{h}$. If we substitute (2.23) and (2.27) into (2.20), we obtain

$$
A_{2 h}=I A_{h} I^{T}
$$

Note, that $A_{2 h}$ and $A_{h}$ are different matrices. However, for the appropriate choice of grids and interpolation operators, equation (2.24) is significantly simpler (i.e. it can be solved faster, because of smaller number of unknowns and coefficients) than the correction equation (2.20) on the fine grid.

Next, the approximate solution of (2.24) can be obtained with the help of some iterative algorithm, which is denoted here as $S_{2 h}\left(A_{2 h}, \vec{r}_{2 h}, 0\right)$.

$$
\vec{\delta}_{2 h}=S_{2 h}^{\prime}\left(A_{2 h}, \vec{r}_{2 h}, 0\right)
$$

After which, the approximate solution of the correction equation is interpolated from the coarse to the fine grid.

$$
\vec{\delta}_{h}=I \vec{\delta}_{2 h}
$$

Once the correction is interpolated on the fine grid, it is added to the current approximate solution on the fine grid.

$$
\vec{x}_{h}^{i+1 / 2}=\vec{\delta}_{h}+\vec{x}_{h}^{i}
$$

While this step helps to reduce low frequency error components, it also induces some additional high spatial frequencies due to the interpolation procedure. But, this additional high frequency error can be effectively reduced by several iterations of the relaxation algorithm on the fine grid.

Note, that operators $S_{h}, S_{2 h}^{\prime}$ and $S_{h}^{\prime \prime}$ can represent different solution methods, e.g. GaussSeidel iterations, Jacobi iterations, conjugate-gradients or exact solver, among others.

$$
\vec{x}_{h}^{i+1}=S_{h}^{\prime \prime}\left(A_{h}, \vec{b}, \vec{x}_{h}^{i+1 / 2}\right)
$$

Hence, we obtain the corrected approximate solution $\vec{x}_{h}^{i+1}$ of equation (2.17).

Interestingly, there is no need to solve (2.24) exactly, since even an approximate solution helps to reduce low spatial frequency components of the error, leading to faster convergence. Nevertheless, if (2.26) is solved exactly, then only the interpolation error defines the convergence rate of the method. Therefore, accurate and efficient interpolation operators are critical for the overall convergence rate.

However, even if the number of the coarse grid points is about a quarter of the number of points in fine grid (e.g. each grid dimension is halved for a 2D problem), generating an approximate solution of (2.24) can still be a problem. Therefore, it is natural to perform the two grid procedure recursively. The same algorithm (2.21) - (2.29) can be applied after (2.26) for $\vec{\delta}$ for increasingly coarser grids until equation (2.20) is simplified to the limit where it is easily solved, e.g. up to one linear equation. Thus, the overall correction scheme literally becomes multi-grid, 

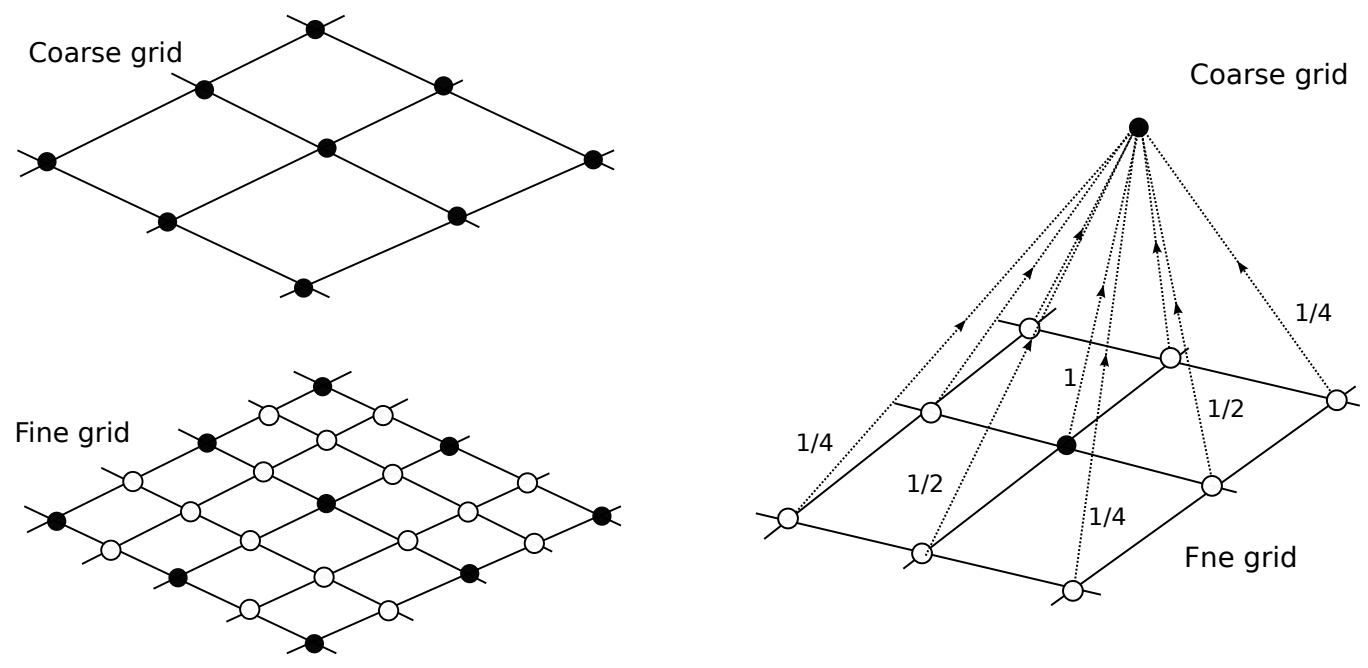

Figure 2.5: Example of grid coarsening (left picture) and inter - grid interpolation with node weights (right picture). The open points from a fine grid are not represented on a coarse grid, however the values of these points are used to interpolate from the fine to coarse grids. The choice of the subset of points for the coarse grid is performed according to a set of heuristic rules (see text for discussion).

since there are many intermediate grids involved.

Each grid level helps to reduce the error in a certain spatial frequency range: the coarsest grid affects the lowest, while fine grid affects highest spatial frequencies. The interpolation between grids introduces some error, but this error is local and, therefore, can be effectively reduced by several iterations of a relaxation algorithm (e.g. $S_{h}$ ), which are performed before and after interpolation steps.

To apply a multi-grid algorithm, we need to define the interpolation operators and the grid hierarchy. There are many possible ways to do it. We consider briefly only geometrical and algebraic approaches.

\subsubsection{Geometrical multigrid}

In the so-called geometrical multi-grid formulation, the interpolation operators are defined heuristically, since a reasonable choice usually works. In the absence of boundaries, the linear interpolation operator can be constructed easily, but some difficulties are typically met near boundaries. Due to the recursive nature of the algorithm, incorrectly interpolating near the boundary reduces the convergence rate significantly.

It should be noted that the support function (i.e. $\xi_{i}(x)$ from (2.11)) from the finite element discretization is continuous, so values that do not fall on grid points can be calculated. It is natural to use this information to define the interpolation operator everywhere on the grid, including boundaries, without resorting to heuristic rules. This method is used in the algebraic multigrid method for finite elements (see discussion below), but for unknown reasons, this approach is not frequently discussed in the textbooks about geometrical multigrid in the context of interpolation 
near the boundaries.

For our implementation of the geometrical method, we choose to use interpolation based on finite elements. With this approach we obtained a good convergence rate.

In multigrid methods, grids and the rules for transforming between different grids must be defined. In the geometrical multigrid formulation, the selection of intermediate grids is performed heuristically from geometrical considerations (see example in Fig. 2.5). For rectangularly structured grids, a popular choice is to reduce grid spacing by a factor of two, but many other approaches are also possible [32]. In many cases, the choice of a factor of two allows superior convergence rates to be obtained. But, this choice leads to severe problems for irregularly spaced or highly anisotropic grids, because the grid properties are not taken into account by the interpolation operator.

Unfortunately, the latter issues with irregularly spaced grids are important for our study. An irregularly spaced grid can be rescaled by factors of two with the help of so-called linesmoothers, i.e. that the iterative relaxation algorithms (e.g. $S_{h}$ from the previous discussion) are applied grid-line-wise instead of point-wise. But, this is overkill in the case where the irregular portion of the grid is small compared to the full grid. A mixed approach, where line-smoothers are applied to a part of the grid only, is very inconvenient, because it requires either, a very robust algorithm, which can classify the grid automatically, or a significant amount of manual classification for every case.

It is also difficult to build a grid hierarchy for domains that have a non-rectangular shape, or for rectangular domains that contain internal structures (like electrodes). For example, if the potential of some white grid points in Fig. 2.5 is fixed, e.g. these points belong to an internal electrode, then the coarse grid cannot be defined by uniformly reducing the grid spacing by a factor of two. This issue can be addressed with the help of the capacity matrix (see below) while keeping the grid rectangular. Such an approach, however, requires solving the Poisson equation twice.

By the capacity matrix, we mean the following: equation (2.17) is linear, therefore it can be inverted, i.e.

$$
\vec{x}=A^{-1} \vec{b}
$$

Let us choose a subset of grid points $\left(\vec{x}_{\text {set }}\right)$, for which we want to keep the potential at a predefined value, i.e. these points represent electrodes.

Due to the linearity of equation (2.30), the potential values at this set of point can be written as follows

$$
\vec{x}_{\text {set }}=\vec{x}_{\text {set }}^{\mathrm{o}}+C \vec{b}_{\text {set }}
$$

Here $\vec{x}_{\text {set }}^{\mathrm{O}}$ is the contribution to $\vec{x}_{\text {set }}$ from all other grid points, $\vec{b}_{\text {set }}$ are the components of the right hand side of equation (2.17) that correspond to $\vec{x}_{s e t}$, and $C$ in the capacity matrix, i.e. $\vec{x}_{\text {set }}=\vec{x}_{\text {set }}^{\mathrm{O}}$ if the charge density in the grid points corresponding to $\vec{x}_{\text {set }}$ is zero.

Hence, if the capacity matrix is found, the algorithm is the following. One should solve Poisson's equation, then find the appropriate $\vec{b}_{\text {set }}$ for the desired potential $\vec{x}_{\text {set }}^{\mathrm{t}}$ in the selected set i.e.

$$
\vec{b}_{\text {set }}=C^{-1}\left(\vec{x}_{\text {set }}^{\mathrm{t}}-\vec{x}_{\text {set }}^{\mathrm{o}}\right),
$$

and then solve Poisson's equation (2.17) once again with the right hand side modified by the 
corrected charge density obtained from (2.32).

\subsubsection{Algebraic multigrid}

An alternative formulation is the so-called algebraic multigrid (AMG) In this approach, the intermediate coarse grids are selected according to some heuristic rules, but, in contrast to geometrical multigrid, the coefficients matrix itself is also taken into account. Because of bulk and complexity of these heuristic rules please refer to the specific literature (e.g. [31,33]) for a review.

As the coarse grid points are selected, it is possible to construct an interpolation operator that minimizes the additional interpolation-induced error on the fine grid. Thus, the grid irregularity and grid locations with bad convergence (e.g. free boundary conditions or the axis in RZ geometry) can be taken into account in interpolation operators, which improves the stability of the method, albeit at the cost of a small decrease in the convergence rate.

In our case, the coefficients matrix in (2.17) comes from the finite element style discretization. For this case there are several recipes for generating an interpolation operator. We choose to use AMG for finite elements (i.e. AMGe), we have not tried other possibilities (such as AMG preconditioned Conjugate Gradients) since the selected method already shows good performance.

The AMGe [34] approach proposes building a set of elements on the base of coarse points. This method offers a procedure to create an efficient interpolation operator, i.e. it induces a small additional error, but it is not very computationally demanding. But, as a downside, it requires that the element information be stored and propagated to different grids. For the first coarse level, propagating the elements does not cause any problems. But, on the subsequent levels, the elements might be required to take on very complicated shapes, and dealing with these shapes is problematic.

Fortunately, there are element-less AMGe methods [35], which allows the interpolation operator to be built from the matrix structure, without explicitly using the elements. Such an interpolation operator has similar qualities to the original AMGe and eliminates the need to deal with some geometrical elements, leading to an algorithm that is simpler to program and support. ${ }^{4}$

We programmed custom implementations of the geometrical multi-grid and element-less AMGe. For the rectangular domain with an irregularly spaced rectilinear grid, our implementation of the geometrical multigrid is faster than our implementation of AMGe. But for domains with internal structures (e.g. probe, grid etc.), the approach of a geometrical multigrid, coupled with a capacity matrix requires solving Poisson's equation twice and multiplication of dense capacity matrix by the part of the solution vector, the perfomance penalties due to these additional steps are typically very significant. Therefore, by default the element-less AMGe solver is used in the program. 


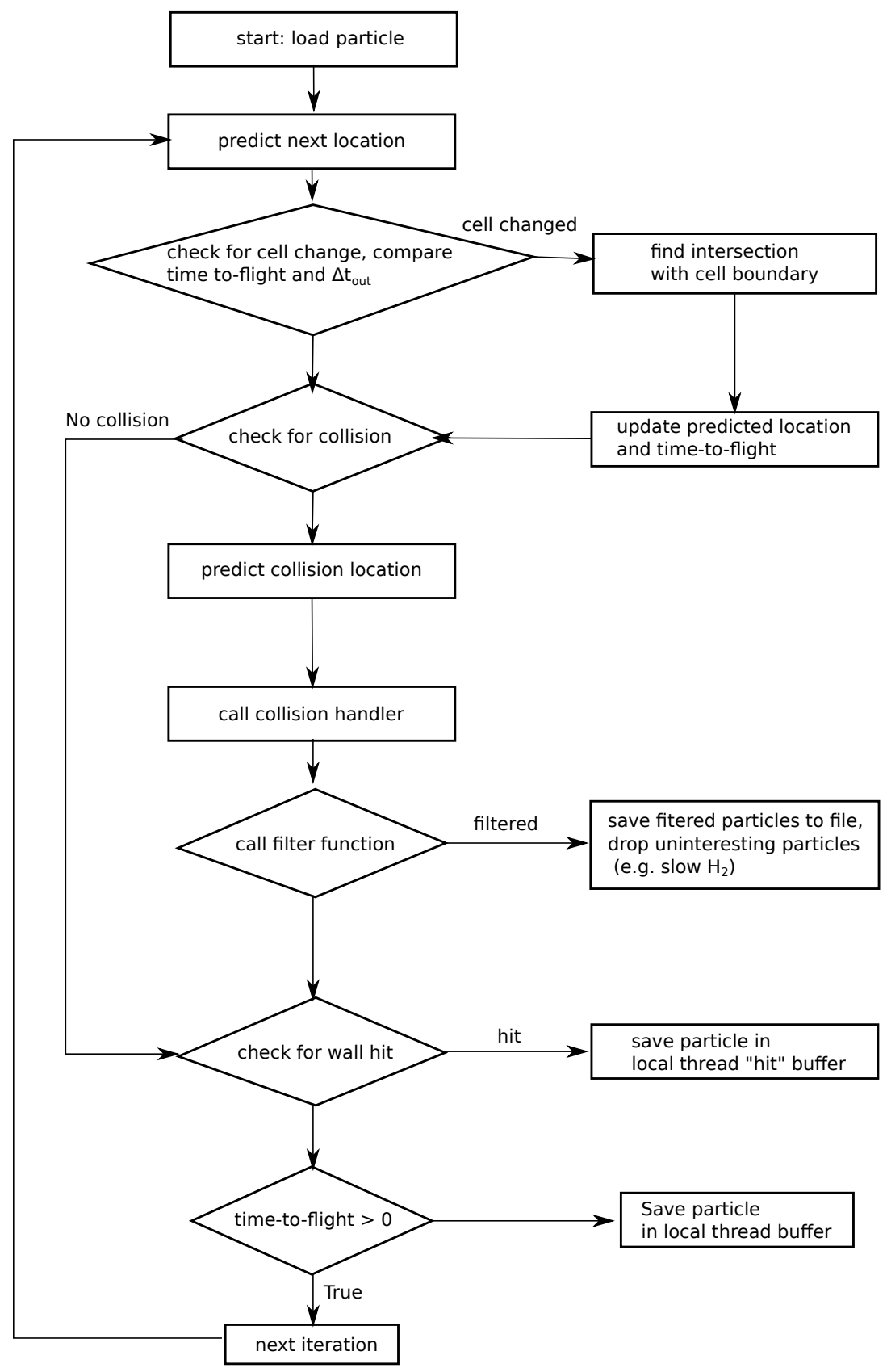

Figure 2.6: Flow chart for particle mover component of the code. The outer loop over all particles is not shown. 


\subsection{Particle mover}

The particle-mover code component of the flow-chart (Fig. 2.3) is presented in Fig. 2.6. Since all components of the code are $2 \mathrm{D} R Z$ it is convenient to rewrite equation (2.14) in $R Z$ coordinates

$$
\begin{aligned}
& \ddot{r}=\frac{v_{\theta}^{2}}{r}-\frac{q}{m} \sum_{j} \alpha_{j} \frac{\partial \xi(r, z)}{\partial r} \\
& \ddot{z}=-\frac{q}{m} \sum_{j} \alpha_{j} \frac{\partial \xi(r, z)}{\partial z} \\
& \frac{\partial\left(r v_{\theta}\right)}{\partial t}=0
\end{aligned}
$$

here $r, z$, and $v_{\theta}$ are the coordinates and tangential component of the velocity of the particle. For clarity, the index that denotes the particle is omitted. Simple time discretization for this equation is used everywhere except near axis cells.

$$
\begin{aligned}
r^{n+1} & =r^{n}+\Delta t v_{r}^{n}+\frac{1}{2} \Delta t^{2}\left(\frac{\left(v_{\theta}^{n}\right)^{2}}{r^{n}}-\frac{q}{m} \sum_{j} \alpha_{j}^{n} \frac{\partial \xi}{\partial r}\left(r^{n}, z^{n}\right)\right) \\
v_{r}^{n+1} & =v_{r}^{n}+\Delta t\left(\frac{\left(v_{\theta}^{n}\right)^{2}}{r^{n}}-\frac{q}{m} \sum_{j} \alpha_{j}^{n} \frac{\partial \xi}{\partial r}\left(r^{n}, z^{n}\right)\right) \\
z^{n+1} & =z^{n}+\Delta t v_{z}^{n}-\frac{1}{2} \Delta t^{2} \frac{q}{m} \sum_{j} \alpha_{j}^{n} \frac{\partial \xi}{\partial z}\left(r^{n}, z^{n}\right) \\
v_{z}^{n+1} & =v_{z}^{n+1}-\Delta t \frac{q}{m} \sum_{j} \alpha_{j}^{n} \frac{\partial \xi}{\partial z}\left(r^{n}, z^{n}\right) \\
v_{\theta}^{n+1} & =v_{\theta}^{n} \frac{r^{n}}{r^{n+1}}
\end{aligned}
$$

In the cells on the axis of symmetry, the terms proportional to $\frac{\left(v_{\theta}^{n}\right)^{2}}{r^{n}}$ can become large, leading to an inaccurate solution. To overcome this issue, instead of equations (2.36), (2.37) and (2.40),

${ }^{4}$ Actually, the element-based variant can be more convenient then element-less variant, if the sequence of grids is constructed from coarsest to finest, which can be the case when an automatic meshing algorithm is used. 
the particle motion is treated in 3D in these cells, i.e.

$$
\begin{aligned}
& x=r^{n}+\Delta t v_{r}^{n} \\
& y=v_{\theta}^{n} \Delta t \\
& r^{n+1}=\sqrt{x^{2}+y^{2}} \\
& v_{x}^{n+1}=v_{r}^{n}-\Delta t \frac{q}{m} \sum_{j} \alpha_{j}^{n} \frac{\partial \xi}{\partial r}\left(r^{n}, z^{n}\right) \\
& v_{r}^{n+1}=\frac{x \cdot v_{x}+y \cdot v_{\theta}^{n}}{r^{n+1}} \\
& v_{\theta}^{n+1}=\frac{y \cdot v_{x}-x \cdot v_{\theta}^{n}}{r^{n+1}}
\end{aligned}
$$

As discussed in section 2.1.1, if the energy conserving scheme is used directly, it produces a lot of noise in the electric field, which may induce numerical heating. To overcome this issue we use sub-stepping. If a super particle ${ }^{5}$ travels over the cell boundary during one time step, this time step is divided into at least two sub-steps, one to travel from the current position to the cell boundary and the other to travel in the other cell.

To implement sub-stepping, each particle has its corresponding time-of-flight, $\Delta t$, which is initially set to the current time step value. For each iteration, the $\Delta t_{\text {out }}$ required for a particle to leave the current cell is calculated by solving equations (2.36) and (2.38) for $\Delta t_{\text {out }}$ with the cell boundaries for $z^{n+1}$ and $r^{n+1}$. If $\Delta t_{\text {out }}>\Delta t$, the moving step is complete, and a sub-step is not required for this particle. For $\Delta t_{o} u t<\Delta t$, the particle crosses a cell boundary. In this case, the particle is first moved to the cell boundary in a step of $\Delta t_{\text {out }}$, followed by a step of $\Delta t^{\prime}=\Delta t-\Delta t_{\text {out }} . \Delta t_{\text {out }}$ and $\Delta t^{\prime}$ are sub time steps.

It is possible that a particle is moving such that it crosses more than one cell boundary during its time-of-flight. Therefore, the procedure described above is iterative: a new $\Delta t_{\text {out }}$ is calculated from the cell boundary, and if $\Delta t^{\prime}<\Delta t_{\text {out }}$, another sub-step is generated. This is repeated until a final step that does not cross a cell boundary is obtained. On completion, the sum of the sub time steps equals the simulation time step value.

During a time step, a particle may hit the wall, or collide with a background gas molecule. Furthermore, fast particles, near the space charge barrier, or plasma sheath region can also gain or lose a significant portion of their energy. Therefore, we check for collisions during each sub time step as indicated in Fig. 2.6.

\subsection{Sources of ionized species}

Since our aim is to describe a radiation induced pulsed plasma, there are two main sources of ionized species in the model: photoionization in the volume, and ions created by the secondary electron flux from the irradiated surface.

In this section we only describe how new super particles are introduced into the model, because the collisions between super particles and the background gas are included via a Monte

${ }^{5}$ In the following text we use "particle" and "super particle" interchangeably. 
Carlo collision scheme (see section 2.7). Therefore, once super particles are added into model, generation and relaxation of different ion species through collisions are calculated by the Monte Carlo scheme.

\section{Photoionization source}

Direct photoionization of hydrogen by EUV photons is included as two processes:

$$
\begin{aligned}
& \mathrm{H}_{2}+h \nu \rightarrow \mathrm{H}_{2}^{+}+e \\
& \mathrm{H}_{2}+h \nu \rightarrow \mathrm{H}^{+}+\mathrm{H}+e
\end{aligned}
$$

The energy resolved cross-sections for these processes are taken from [36].

The EUV pulse has a certain temporal profile, spatial profile and spectral distribution. Therefore, the number of photoionization events in the volume is computed for each photoionization process in advance, for a given EUV emission spectrum, according to the integral cross-section. At each time step we only need to sample the number of photoionization events, weighted by the temporal and spatial profiles of EUV pulse, while the photon energy is sampled according to the EUV spectral profile.

The energy of emitted electrons is given by the difference between sampled photon energy and the ionization potential of molecular hydrogen. In turn, the corresponding ions are cold, because of the large mass ratio between molecular hydrogen and the electron. The velocity direction of each emitted "super" electron (in the sense of distribution (2.9)) is sampled according to a uniform angular distribution.

For the considered pressure range, the gas is almost transparent. For a photon energy of $92 \mathrm{eV}$ and a background pressure of $22 \mathrm{~Pa}$, one obtains that $N_{H_{2}} \times \sigma_{\text {ion }}=3 \cdot 10^{-4} 1 / \mathrm{cm}$.

\subsubsection{Secondary electron emission source}

The irradiation of any material by EUV leads to photoelectron emission, since, for most materials, the work function $(W)$ is about several $\mathrm{eV}$. The emitted electrons are a mixture of primary (i.e. $E_{\text {electron }}=E_{\text {photon }}-W$ ), and secondary electrons. Secondary electrons (SEs) undergo several inelastic collisions in the solid before emission, thus, the secondary electron energy spectrum is broad.

The yield and spectrum of SEs depends significantly on the surface conditions [37, 38], since the absorption length of EUV is highly material dependent as are the scattering lengths of electrons generated by EUV absorption. Furthermore, the emission of secondary electrons is highly dependent on the energy and occupation of surface electronic states, which are heavily influenced by the physical make up of the surface (e.g., contaminant layers), and the depth dependence of the primary electron generation. Thus, the SEY and SEE are very difficult to predict purely on the basis of materials and cross sections. We choose to avoid this by treating SEE and SEY as free parameters.

For many materials, the energy spectrum of photoelectrons, emitted from the surface due to 
EUV irradiation, can be approximated as [39]:

$$
P(E) \sim \frac{E}{(E+W)^{4}}
$$

Here $P(E)$ is the probability to emit an electron with energy $E$, from a surface with a work function, $W$. For carbon $W=5 \mathrm{eV}$ [40]. For the electrons emitted from the surface, we assume an angular dependence given by a cosine emission law [39].

Because the exact surface conditions are not known and the SE yield is a free parameter, in our simulations, we do not observe ${ }^{6}$ any significant difference if, instead of (7.3), we use a measured SE spectrum taken from [37].

\subsubsection{Particle source}

To aid the process of testing the code, and to provide for vacuum emission, or other arbitrary ion/electron sources, an arbitrary particle generator is also included. This source samples particles according to predefined spatial, temporal, and velocity distributions without any relation to other physical parameters.

\subsection{Collisions with background gas}

Let us return to the right-hand side of equation (2.5), which describes collisions. The general form of the collisional term is complicated, but, fortunately, we do not need to use it. For our case, only binary collisions of electrons, ions, and fast neutrals ${ }^{7}$ with molecules from the background gas should be considered, because electron - electron, electron - ion, and ion - ion Coulomb collisions have a low probability, due to the low plasma density. For the same reason, three body collisions are neglected. Therefore, to introduce collisions, we follow a Monte-Carlo collision scheme [28].

There are many different types of collisions possible, e.g. electron induced ionization, excitations, elastic scattering, etc. Hence, the important part of the task is not only to determine if a collision happened, but also to determine which reaction path (i.e. channel) is followed. Let $\sigma_{k}(v)$ be the cross-section of the $k$ th collision channel from a total of $N$, then the average collision frequency for this channel is $\nu_{k}=\left\langle\sigma_{k}(v) v\right\rangle n$, here $n$ is the concentration of background gas, and $v$ is the relative velocity between gas molecules and a super particle, and angled brackets mean averaging over the velocity distribution.

Since we aim to describe such features as the collisional plasma sheath or space charge potential barrier, we should take into account the change of the collision frequencies when particles gain or loose energy due to variation of potential along their trajectory between collisions. The characteristic time scale for EUV plasma evolution is the EUV pulse duration, which is about

${ }^{6}$ Note that the shape of measured spectrum in [37] might be distorted by amplification, since similar distortion is described in [39]. This can explain why we do not observe an increase in the accuracy of our simulations when we sample SEs according to a measured distribution.

${ }^{7}$ The filter function (see Fig. 2.6) is used to control whether fast neutrals are considered in the same simulation as all other particles or saved to a file. In the latter case, the fast neutrals that were saved to the file are processed after the main simulation by the same code, but with large time steps and with disabled code paths for solving Poisson equation (see Fig. 2.3). 
$100 \mathrm{~ns}$, but the maximum collision frequency for electron impact ionization in $3 \mathrm{~Pa} \mathrm{H}_{2}$ is about $4 \cdot 10^{7} 1 / \mathrm{s}$. Hence, we need to sample collisions, not according to the inverse collision frequency, but according to the simulation time step, since, during several time steps, the energy of a particle can change significantly.

Let $\Delta t$ be the collision sampling interval, such that $\Delta t \ll 1 / \nu_{k}$ for any reaction channel. Then, the collision probability for the $k$ th channel is $p_{k}=n \sigma_{k}(v) v \Delta t$. Note, that there is no need to compute the average $\left\langle\sigma_{k}(v) v\right\rangle$. Instead it is enough to sample the velocity of the target particle according to a Maxwellian distribution and compute the relative velocity of the projectile.

Hence, the probability for one super-particle to undergo a collision during the collision sampling interval is

$$
P=\sum_{k} p_{k}=n \sum_{k} \sigma_{k}(v) v \Delta t
$$

If the choice of sampling time interval is correct, then $P<1$ and the full probability can be rewritten as $1 \equiv(1-P)+P$. The part of interval $(0,1)$ with length $(1-\mathrm{P})$ corresponds to the case of no collision, and the rest of interval corresponds to the case with a collision. To determine if a collision happened or not we only need to sample from a uniformly distributed set of random numbers and test whether the sampled value falls into collisional part of the interval $(0,1)$.

Despite this approach's simplicity, many different optimizations are possible. These optimisations redistribute the intervals, which correspond to collision/no collision, over the interval $(0,1)$. This speeds up looking up collision events (see Fig. 2.7). There are at least two popular schemes to determine if a collision occurs. Let us briefly outline the ideas behind these methods.

The Null-collision method [41] implies that for all possible channels, a "no collision" channel is added. The motivation for adding null reaction channel is to make the probability to undergo $\mathrm{N}+1$ collisions independent of the projectile velocity. The following condition holds (for correctly chosen $\Delta t$ ):

$$
\max _{v}(P)=\Delta t \cdot n \max _{v}\left(\sum_{k} v \sigma_{k}(v)\right) \leq 1,
$$

in many situations, a much stronger condition holds: $\max _{v}(P) \ll 1$. Therefore, let us construct an additional reaction channel which corresponds to "no collision"

$$
p^{\prime}=\max _{v}(P)-P
$$

then, the probability to undergo this $(\mathrm{N}+1)$ collisions during time interval, $\Delta t$, is independent of the particle velocity.

This method can be used to speed up the collision look-up, since, in the first place, the random number should be compared to a precomputed constant $\max _{v}(P)$, and the most frequent result is that no collision happened.

While the Null-collision procedure is simple and straightforward, it requires that all partial probabilities are evaluated to find the proper reaction channel in the case of a collision. This implies a performance penalty, since collisional cross-sections are, typically, tabulated. Thus, the data from many tables should be examined for each collision event. Because the number of super-particles in a typical simulation is large (e.g. $10^{6}$ ), this performance penalty is likely to be 
significant. Moreover, we need to deal with hydrogen, where the number of possible channels is large.

Therefore, we chose the Nanbu method [42], because this method allows a reaction channel to be chosen first and, subsequently, only the partial probability for this reaction channel must be evaluated. The latter is very convenient, because, in the case of slow ions, the velocity distribution of the background gas should be also be taken into account.

As previously, let $p_{k}$ be the probability of the $k$ th collision channel from a total of $N$ available channels, then

$$
1 \equiv 1-\sum_{k=1}^{N} p_{k}+\sum_{k=1}^{N} p_{k}=\sum_{k=1}^{N}\left\{\left(\frac{1}{N}-p_{k}\right)+p_{k}\right\}
$$

The result of such reordering is presented in Fig. 2.7. The reaction channel and probability can be sampled easily, if $\Delta t$ is chosen in such a way that all $p_{k}$ are smaller than $1 / N$.

Let $r$ be drawn from a set of random numbers, uniformly distributed over $(0,1)$, then the channel number $k=$ floor $(r \cdot N)$. The collision occurs if $r-k / N<p_{k}$.

\section{Null collision}

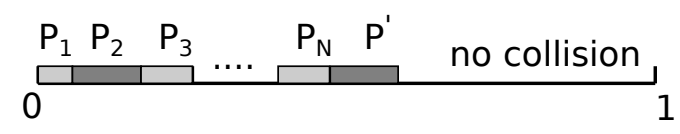

\section{Nanbu scheme}

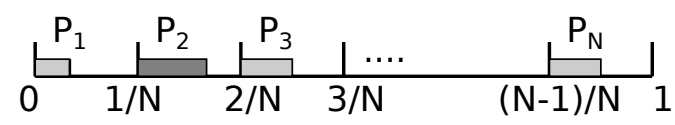

Figure 2.7: Example of the application of scheme [42] compared to the Null collision scheme. The results of sampling the reaction probability with help of random numbers are the same in both cases, but the Nanbu scheme allows to select the appropriate reaction channel efficiently.

The limiting time step for this method is

$$
\Delta t_{\max }=\frac{1}{N \cdot n \cdot \max _{k, v}\left(\sigma_{k}(v) v\right)}
$$

which is much larger than the PIC time step appropriate for low pressure, low density plasmas.

\subsection{Cross-section set and swarm parameters}

The desired property of a low pressure plasma model is a description of experimental results in terms of measurable quantities. However, the accuracy of the conclusions drawn from numerical modelling depends on the reliability of the input parameters, which in our case, is the set of cross-sections for the different channels. 
There are many cross-sections compilations (e.g. [43, 44, 45, 46]), but their use is not straightforward. In experiments, the separation between different reaction channels is not perfect, creating systematic errors in experimentally obtained cross-sections. These have a tendency to accumulate when many measurements, obtained from different experiments, are combined to generate a set of cross-sections.

Hence, many published sets $[47,48,49,50]$ are fine tuned. The fine tuning is not only intended to reduced the errors from combining cross-sections from different sources, but also to compensate for model specific inaccuracies. For example, by treating collision kinematics differently, one may obtain significantly different results with the same set of cross-sections. This leads to incompatibility between the cross-section sets from models based on Monte Carlo collision events, and models based on distribution function dipole (or multipole) decomposition.

Therefore, the standard necessary condition ${ }^{8}$ for the combination of a cross-section set and a model is that the swarm parameters (e.g. ionization coefficient, drift velocity etc.) are predicted by the simulation with reasonable accuracy under relevant conditions.

\subsubsection{Swarm experiment}

Let us describe, in brief, the swarm experiment (for review please refer to specific literature, e.g. $[52,53])$. A small number of ions (or electrons) are injected into a gas filled tube that has an external bias applied over its length. The charged particles are accelerated along the tube, and, depending on the applied bias, generate more charged particles. These charged particles arrive at the end of the tube, generating a pulse of current in the electrode. The current is measured as a function of time, pressure, and bias. The measured time for the current pulse to arrive at the electrode, and the current pulse's magnitude allow the average drift velocity, average energy, ionization coefficient, and many other parameters to be determined.

The main advantage of the experiment is it's simplicity - it is hard to get it wrong. The small number of injected particles guarantees that the experiment operates in a linear regime with negligible effects from charged particle Coulomb force interaction, therefore, the reduced field strength (i.e. $E / N, E$ - the field strength, $N$ - the concentration of background gas) is a well defined quantity. Most importantly, the large body of swarm experimental data from different experimental groups agrees well when experimental conditions are comparable (e.g., for the same combination of ion, gas, and reduced field strength).

We tested the consistency of our implementation by modeling swarm experiments and found good agreement between the simulation output and experimental values [54] for electron ionization coefficients, electron mobility, and for $\mathrm{H}^{+}$and $\mathrm{H}_{3}^{+}$mobility in hydrogen [55]. These results tell us that the combination of the employed set of cross-sections and our model provides reasonably accurate physical results and can be relied upon over the parameter range that we explore in chapter $5-7$.

\subsubsection{Cross-section set}

The cross-sections set used in the present model consists of electron collisions with hydrogen, ion collisions with hydrogen and several neutral-neutral collisions to keep track of fast neutrals.

\footnotetext{
${ }^{8} \mathrm{An}$ interesting discussion and a comparison of cross-section sets is presented in [51]
} 
Table 2.1: Electron $-\mathrm{H}_{2}$ cross-sections set used in model.

\begin{tabular}{|c|c|c|c|c|}
\hline & \multirow[t]{2}{*}{ reaction } & \multirow{2}{*}{$\begin{array}{c}\text { energy loss } \\
\mathrm{eV}\end{array}$} & \multicolumn{2}{|c|}{ reference } \\
\hline & & & integral & differential \\
\hline & elastic scattering & & & \\
\hline 1) & $\begin{array}{l}e+H_{2} \rightarrow e+H_{2} \\
\text { ionization }\end{array}$ & - & [46] & [46] \\
\hline 2) & $e+H_{2} \rightarrow e+e+H_{2}^{+}$ & 15.426 & [56] & {$[57,58]$} \\
\hline 3) & $e+H_{2} \rightarrow e+e+H_{2}^{+*} \rightarrow e+e+H^{+}+H$ & 18.1 & {$[45]$} & \\
\hline 4) & $\begin{array}{l}e+H_{2} \rightarrow e+e+H_{2}^{+*} \rightarrow e+e+H^{+}+H \\
\text { vibrational excitations }\end{array}$ & 30.6 & [45] & \\
\hline 5) & $e+H_{2}(v=0) \rightarrow e+H_{2}(v=1)$ & 0.516 & {$[59,44]$} & \\
\hline 6) & $e+H_{2}(v=0) \rightarrow e+H_{2}(v=2)$ & 1.0 & [59] & \\
\hline 7) & $\begin{array}{l}e+H_{2}(v=0) \rightarrow e+H_{2}(v=3) \\
\text { rotational excitations }\end{array}$ & 1.5 & [59] & \\
\hline 8) & $e+H_{2}(j=0) \rightarrow e+H_{2}(j=2)$ & 0.044 & [60] & \\
\hline 9) & $\begin{array}{l}e+H_{2}(j=1) \rightarrow e+H_{2}(j=3) \\
\text { electronic excitations }\end{array}$ & 0.077 & [60] & \\
\hline 10) & $e+H_{2} \rightarrow e+H_{2}\left(B^{1} \Sigma_{u}^{+}\right)$ & 12.75 & [45] & [46] \\
\hline 11) & $e+H_{2} \rightarrow e+H_{2}\left(C^{1} \Pi_{u}\right)$ & 13.29 & [45] & [46] \\
\hline 12) & $e+H_{2} \rightarrow e+H_{2}\left(a^{3} \Pi_{u}\right)$ & 11.72 & [45] & [46] \\
\hline 13) & $e+H_{2} \rightarrow e+H_{2}\left(b^{3} \Sigma_{u}^{+}\right) \rightarrow e+H+H$ & 7.93 & [45] & \\
\hline 14) & $e+H_{2} \rightarrow e+H_{2}\left(c^{3} \Pi_{u}\right)$ & 11.72 & [45] & [46] \\
\hline 15) & $e+H_{2} \rightarrow e+H_{2}\left(d^{3} \Pi\right)$ & 13.6 & [45] & \\
\hline 16) & $e+H_{2} \rightarrow e+H_{2}\left(e^{3} \Pi\right)$ & 13.0 & [45] & \\
\hline 17) & $e+H_{2} \rightarrow e+H_{2}(L \alpha)$ & 15.0 & {$[47]$} & \\
\hline 18) & $e+H_{2} \rightarrow e+H_{2}$ (Rydberg. Sum) & 15.2 & {$[61,47]$} & \\
\hline
\end{tabular}

The list of processes included in the model is presented in Table.2.1 and Table.2.2.

The set for electron - neutral collisions is based on the set of [64]. Despite this set having a lot in common with an older set from [48], the consideration of the differential cross-section data significantly affects the simulation results and allows the behavior of the ionization coefficients to be reproduced with better accuracy for high values of the reduced electric field (i.e. $>1000 \mathrm{Td})$.

The set for ion - neutral collisions is based on [62], with the inclusion of more recent measurements for $\mathrm{H}_{3}^{+}$destruction (e.g. reactions 13, 14, 15, 16 in Table 2.2) by [63] as indicated in remarks from [50]. Note, that there is some discussion in [62] about the dependence of the cross-section for $\mathrm{H}_{3}^{+}$destruction on the internal rovibrational state of $\mathrm{H}_{3}^{+}$. Therefore, the ion composition for the high energy part of the energy distribution function of the ion flux to a surface, which is computed in this work, could have a significant systematic error.

The set for neutral - neutral collisions is the most basic and is based on [48]. These processes are included because it could be important in experiments with an external bias. $\mathrm{H}_{3}^{+}$ions are accelerated by the external field towards a sample, but a significant fraction of $\mathrm{H}_{3}^{+}$ions can be destroyed in collisions. Therefore, it is possible that a directed flux of fast neutrals is produced (e.g. reaction 11 and 16 in Table 2.2). If neutral - neutral collisions are not taken into account 
Table 2.2: Ion $-\mathrm{H}_{2}$ cross-sections set used in model.

\begin{tabular}{|c|c|c|c|}
\hline & reaction & $\begin{array}{l}\text { energy loss } \\
\mathrm{eV}\end{array}$ & reference \\
\hline & momentum transfer & & \\
\hline 1) & $\begin{array}{l}H^{+}+H_{2} \rightarrow H^{+}+H_{2} \\
\text { rotational excitations }\end{array}$ & - & {$[62]$} \\
\hline 2) & $H^{+}+H_{2}(j=0) \rightarrow H^{+}+H_{2}(j=2)$ & 0.044 & {$[62]$} \\
\hline 3) & $\begin{array}{l}H^{+}+H_{2}(j=1) \rightarrow H^{+}+H_{2}(j=3) \\
\text { vibrational excitations }\end{array}$ & 0.077 & {$[62]$} \\
\hline 4) & $H^{+}+H_{2}(v=0) \rightarrow H^{+}+H_{2}(v=1)$ & 0.516 & {$[62]$} \\
\hline 5$)$ & $H^{+}+H_{2}(v=0) \rightarrow H^{+}+H_{2}(v=2)$ & 1.0 & {$[62]$} \\
\hline 6) & $\begin{array}{l}H^{+}+H_{2}(v=0) \rightarrow H^{+}+H_{2}(v=3) \\
\text { charge transfer }\end{array}$ & 1.5 & {$[62]$} \\
\hline 7) & $\begin{array}{l}H^{+}+H_{2} \rightarrow H+H_{2}^{+} \\
H_{3}^{+} \text {formation }\end{array}$ & - & [62] \\
\hline 8) & $\begin{array}{l}H_{2}^{+}+H_{2} \rightarrow H_{3}^{+}+H \\
\text { charge transfer }\end{array}$ & - & [48] \\
\hline 9) & $\begin{array}{l}\mathrm{H}_{2}^{+}+\mathrm{H}_{2} \rightarrow \mathrm{H}_{2}^{+}+\mathrm{H}_{2} \\
\text { vibrational excitation }\end{array}$ & - & [48] \\
\hline 10) & $\begin{array}{l}H_{2}^{+}+H_{2} \rightarrow H_{2}^{+}+H_{2} v 1 \\
\text { collision induced dissociation }\end{array}$ & 0.516 & [48] \\
\hline 11) & $\begin{array}{l}H_{2}^{+}+H_{2} \rightarrow H_{2}^{*+}+H_{2} \rightarrow H^{+}+H+H_{2} \\
\text { momentum transfer }\end{array}$ & 2.65 & {$[48]$} \\
\hline 12) & $\begin{array}{l}H_{3}^{+}+H_{2} \rightarrow H_{3}^{+}+H_{2} \\
\text { charge transfer }\end{array}$ & - & {$[62]$} \\
\hline 13) & $H_{3}^{+}+H_{2} \rightarrow H_{3}+H_{2}^{+*} \rightarrow H_{2}+H+H^{+}+H$ & - & {$[63]$} \\
\hline 14) & $\begin{array}{l}H_{3}^{+}+H_{2} \rightarrow H_{3}+H_{2}^{+} \rightarrow H_{2}+H+H_{2}^{+} \\
\text {collision induced dissociation }\end{array}$ & - & {$[63]$} \\
\hline 15) & $H_{3}^{+}+H_{2} \rightarrow H_{3}^{+*}+H_{2} \rightarrow H^{+}+H_{2}+H_{2}$ & - & {$[63]$} \\
\hline 16) & $\begin{array}{l}H_{3}^{+}+H_{2} \rightarrow H_{3}^{+*}+H_{2} \rightarrow H_{2}^{+}+H+H_{2} \\
\text { momentum transfer }\end{array}$ & - & {$[62]$} \\
\hline 17) & $H+H_{2} \rightarrow H+H_{2}$ & - & {$[48]$} \\
\hline 18) & $H_{2}+H_{2} \rightarrow H_{2}+H_{2}$ & - & {$[48]$} \\
\hline
\end{tabular}


this directed flux of neutrals will be considerably overestimated.

\subsubsection{Differential cross-sections}

Most electron - neutral [46] as well as some of ion - neutral [65] collisions are anisotropic, with the anisotropy depending on the impact velocity. The anisotropy is not so important for large isotropic plasmas, since the anisotropy is averaged by many collisions. Unfortunately, we need to deal with the situation where a preferred direction exists due to an external bias, and with physical dimensions that are comparable to the collisional mean free path. Under these conditions, anisotropic effects could be large.

In addition to anisotropy and as described in detail in [64], the particular choice of the differential cross-sections significantly influences the simulation results for the ionization coefficient and the drift velocity. For example, in our simulations of the swarm experiment, if all other parameters are frozen, the variation of the energy distribution function for electrons, ejected during ionization could change the value of the simulated ionization coefficient by $30 \%$ for $\mathrm{E} / \mathrm{N}$ $\sim 1000 \mathrm{Td}$. This is a significant effect, since, in many cases, the simulated current depends exponentially on the ionization coefficient.

Therefore, we use the differential cross-section data, if available, to perform computations of the collision kinematics. The list of types of collision kinematics used in the model is presented in Section 2.8.4. This choice, however, has a cost. On the one hand it allows collisions to be described more accurately, but, on the other hand, the use of the differential cross-section data significantly expands the parameter space of the model. Hence, it makes it very difficult to compare sets of cross-sections from different models, because the results depend on the details of the computational model for the kinematics, which are rarely presented in detail.

\subsubsection{Collision types}

After a particular collision channel is selected, kinematic calculations should be performed. Fortunately, it is possible to classify all collisions into a small number of corresponding classes.

- ELECTRON SCATTER. For elastic electron - neutral processes there is no need to compute the relative velocity because the mass difference between the electron and the neutral is very large. The kinematic computation only has to select a new random velocity according to some distribution function.

- ELECTRON EXCITATION. This is almost the same as the case for ELECTRON SCATTER, but energy loss due to excitation is included.

- ELECTRON IONIZATION. For the correct description of the ionization process, the energy distribution of the ejected electrons must be taken into account. Thus, the kinematic computation is more complicated, compared to ELECTRON EXCITATION.

- TWO PARTICLE. Solution of a two body scattering problem with optional energy loss due to excitations, and ionzation, etc. The main difference from ELECTRON EXCITATION is that, here, we compute the final state for both interacting particles. 
- TWO PARTICLE FORWARD. For many processes, the relevant differential cross-sections are not available, but it is known that the reaction products are directed forward in the center of mass reference frame. Therefore, for this processes, we solve the two body problem, include energy loss if any, but the scattering kinematics correspond to forward scattering in the center of mass reference frame.

- CHARGE TRANSFER. Resonant charge transfer collision is backward scattering in the center of mass reference frame. This is very efficiently modeled by swapping particle velocities, i.e. no random numbers.

- COLLISION INDUCED DISSOCIATION. After collision induced dissociation, the particles are deflected by very small angles. Therefore, this situation is modeled as two body scattering with frozen velocity directions in the center of mass reference frame.

Please note, that it is natural to describe the kinematics of some reactions (e.g. 13, 14, 15, 16 from Table 2.2) in two steps, where the intermediate unstable products (e.g. $\mathrm{H}_{3}$ ) are formed after the first step. These unstable intermediate particles are immediately converted to stable products according to certain branching ratios. This approach has two benefits (a) it reflects the physics of the processes, and (b) it significantly simplifies the kinematic computations, since at each step, at most, only the two body problem should be considered. That is far simpler than accurately sampling a three (or more) body final state.

\subsection{Ion induced electron emission}

For many combinations of target material and ion type, the electron yield for ion induced electron emission as function of incoming ion energy is characterized by two distinct regions. For slow ions, the electron yield does not depend on the ion energy, but depends on the ion ionization potential, and target material's work function [66,67]. This regime is often referred to as potential emission (PE).

In the model, the PE probability to emit an electron per ion impact at the bottom electrode is computed with the following semi empirical formula from [67]

$$
\gamma_{P E} \approx \frac{0.2}{E_{F}}\left(0.8 E_{i}-2 W\right)
$$

Here $E_{F}$ is the metal Fermi energy, $E_{i}$ is the ionization energy of the ion and $W$ is the metal's work function. The applicability range of (5.1) is $3 W \leq E_{i} \leq\left(2 E_{F}+W\right)$, the accuracy of this approximation in this range is not better than $10 \%$. Outside this range, the accuracy of (5.1) slowly decreases.

In the fast ion regime, the electron yield strongly depends on the incoming ion energy, this is frequently referred to as kinetic induced emission (KE). For our conditions, the relevant ion energy range is below $0.5 \mathrm{keV}$. For this range, the KE probability was measured experimentally for $\mathrm{H}^{+}$ions, incident on a gold target [68]. The results clearly shows the transition from $\mathrm{PE}$ to $\mathrm{KE}$ region, and that the magnitude of $\mathrm{PE}$ is in agreement with that estimated using (5.1).

There are KE models [69], but, they are complicated and require some fitting parameters to be defined. Since, in our case, the exact surface conditions are not known well, the implementation 
of a KE model offers no benefit over fitting to the experimental data. Therefore, we follow the same approach as in [70] $]^{9}$, and uses a function that is fit to the experimental data [71] for KE for all metallic surfaces.

$$
\gamma_{K E} \approx 6.2 \cdot 10^{-5} \cdot(E[\mathrm{eV} / \mathrm{amu}])^{1.15}
$$

Here $E$ is ion kinetic energy (in $\mathrm{eV}$ ) per unit mass (in $\mathrm{amu}$ ) of the ion.

The physical motivation for this approach is the following. For many metals and conductive materials, the work function $(W)$ is approximately $5 \mathrm{eV}$ and the quasi-classical threshold of KE is $v_{i}>W /\left(2 k_{F}\right)$, where $v_{i}$ is the ion velocity, and $k_{F}$ is the Fermi impulse. KE depends strongly on the ion type and the target material, especially for heavy ions. But, in experiments [71] the KE probability as a function of velocity, varies only by a factor of two or less for $\mathrm{H}^{+}, \mathrm{H}_{2}^{+}$and $\mathrm{H}_{3}^{+}$for a gold target. Furthermore, in other experiments [72] the same ion species show quite similar KEs for a broader range of target materials.

Unfortunately, for simulations where ion induced electron emission is a major effect (i.e. stable glow discharge), the accuracy of these approximations is not sufficient. For such cases, PE and KE yields should be considered as free parameters with a variation in the range of at least about a factor of two. Nevertheless, we include these estimates in the model, since we need to simulate sheath dynamics in a pulsed plasma. For this situation, the inclusion of PE and KE is motivated by the physics. Furthermore, it increases the accuracy of results, because the above formulas, in many cases, provide quite reasonable estimates.

\subsection{Displacement current}

The displacement current is also included in the model. It is computed at each time step as the numerical derivative of the electric field over each of the electrode boundaries. The inclusion of the displacement current allows the simulated and experimentally measured discharge currents to be compared.

${ }^{9}$ For some reason, PE is not included in [70], and KE is approximated as a fit function of ion energy for all hydrogen ions. In our case, the KE fit used in the present work differs from one presented in [70]. 



\section{Chapter 3}

\section{Test problems}

To test our implementation, we simulated several analytically solvable problems. Special attention was paid to simulations of a vacuum diode in conditions which were far from the saturation regime, because these simulations provide a benchmark for a situation with significant space charge effects.

Note, that one of the benefits of these simulations is the overall model integrity check, since the analytical solution not only predicts the shape of IV characteristic, but also predicts the absolute current value.

\section{1 "Cold" diode}

This simulation is intended to reproduce the well known 3/2 law for current density - bias (IV) characteristics of a vacuum diode when the initial electron energy is negligible [73].

$$
i=\frac{1}{9 \pi}\left(\frac{2 q_{e}}{m_{e}}\right)^{3 / 2} \frac{V^{3 / 2}}{d^{2}}
$$

Here $i$ is the current density, $q_{e}$ and $m_{e}$ are the electron charge and mass, $V$ is the external bias, and $d$, the distance between the electrodes. The configuration of the simulation is presented in Fig. 3.1. To avoid boundary $2 \mathrm{D}$ effects (the $3 / 2$ law is derived for a $1 \mathrm{D}$ case), the region where the current density is sampled is smaller than the electron source.

The comparison between the simulation results and the analytical solution is presented in Fig. 3.2. The systematic error in the simulation is small and mostly induced by the fact that, for the case of an initial electron velocity of exactly zero, we would need to use a time step that was impractically small, and a grid resolution that is too high.

\section{2 "Hot" diode}

The "cold" diode case, presented above, is not sensitive to the initial energy distribution of the electrons. Fortunately, there is an analytical solution [74] for a diode where electrons are emitted with a Maxwellian distribution function in the direction normal to the electron source.

$$
d N_{e} \sim v_{z} \exp \left(-\frac{m_{e} v_{z}^{2}}{2 K T_{e}}\right) d v_{z}
$$

Despite the fact that this EDF differs from (7.3), it allows the model's response as function of the initial electron temperature to be tested. 


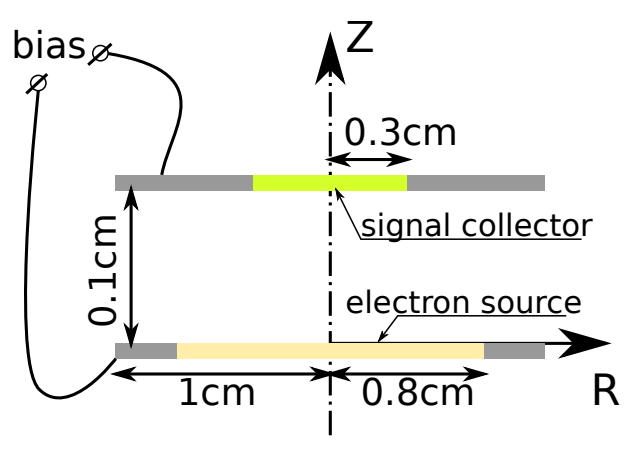

Figure 3.1: Sketch of configuration for diode simulations.

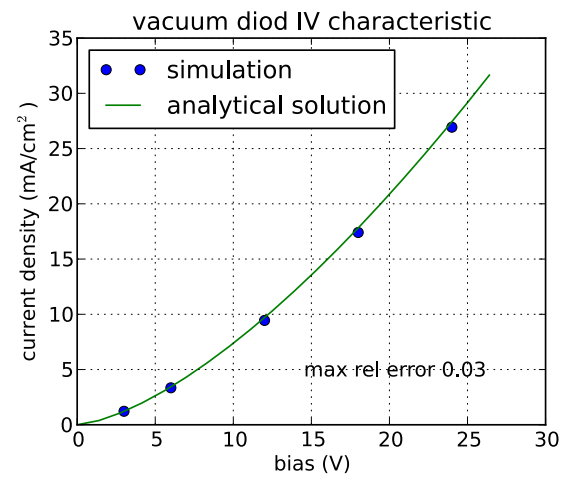

Figure 3.2: Comparison between simulated and analytical IV characteristics for a vacuum diode.

The analytical solution has a form defined by (3.3) - (3.10). The input parameters are (in CGS units): the saturation current, $I_{0}$, and the current density, $I$, and the distance between the electrodes, $d$. A set of equations must then be solved to find $V$ for a given $I$. The solution starts from determining $V_{m}$, the space charge barrier height, and its position, $X_{m}$. After which, a reduced dimensionless potential $(\eta)$ is calculated, and the minimum of the reduced potential, $\eta_{m}$ is used to calculate the bias (3.10).

$$
\begin{gathered}
V_{m}=-K T_{e} / q_{e} \ln \left(\frac{I_{0}}{I}\right) \\
L=2 m_{e}^{1 / 4}\left(\frac{\pi}{2 K T}\right)^{3 / 4} \sqrt{I \cdot q_{e}} \\
\xi_{m}(\eta)=\int_{0}^{\eta} \frac{d x}{\sqrt{\exp (x)-1+\exp (x) \cdot \operatorname{erf}(\sqrt{x})-\frac{2 \sqrt{x}}{\pi}}} \\
\xi_{p}(\eta)=\int_{0}^{\eta} \frac{\xi_{m}=\frac{\xi_{m}\left(\ln \left(I_{0} / I\right)\right)}{2 L}}{\sqrt{\exp (x)-1-\exp (x) \cdot \operatorname{erf}(\sqrt{x})+\frac{2 \sqrt{x}}{\pi}}} \\
\xi_{p}\left(\eta_{m}\right)=\xi_{t} \\
V=V_{m}+\frac{K T_{e}}{q_{e}} \eta_{m}
\end{gathered}
$$

For derivation and discussion of this solution please refer to publication [74].

Therefore, we performed simulations with the same grid and time step, while the initial 


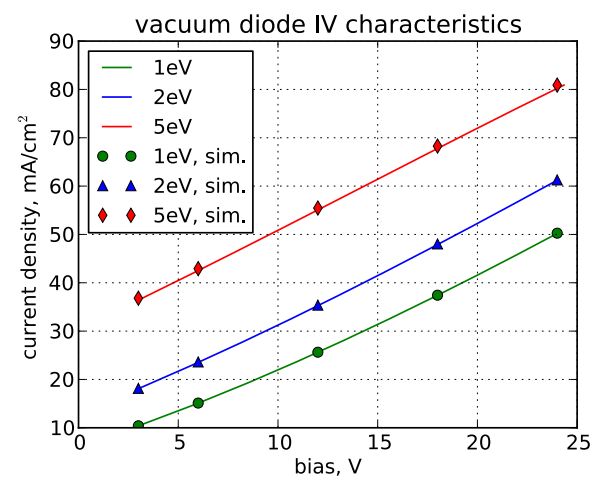

Figure 3.3: Comparison between simulated and analytically calculated IV characteristics for cases with an initial Maxwellian EDF.

value of $T_{e}$ was varied. Comparison between the simulated IV characteristics and the analytical solution is presented in Fig. 3.3.

These simulations show that, even in cases where electrons are allowed to travel more than one cell per time step (1.5 cells on average), the IV characteristic is reproduced correctly due to sub-stepping. However, for very large time steps, where electrons, on average, travel more than two cells, the solution's accuracy deteriorates significantly. This was expected, since sub stepping is a method to fix problems with numerical heating for a minority of fast particles. Sub stepping works well if the fast minority of particles, which can travel many cells per time step, do not affect the potential distribution in the simulation domain. If this condition is violated, the time step should be decreased. 



\section{Chapter 4}

\section{Research goal and experiments selection}

The goal of the present work, as discussed in section 1.1, is to calculate the composition and energy distribution of the ion flux from the EUV induced plasma to the surface. Due to the pulsed nature of the plasma, the flux depends on the dynamics of the plasma, i.e. ignition of the plasma by EUV radiation and secondary electrons, and the plasma's subsequent decay.

To simulate the plasma, a number of parameters are required, e.g. EUV pulse energy, EUV spectrum, temporal profile of the EUV pulse, SE yield, SE spectrum, cross-sections set etc. Unfortunately, many of these parameters are known with poor accuracy and some of them depend significantly on the experimental setup. Moreover, the model has it own unknown error margin, since the transient behavior of the model was not checked by the tests, described in Chapter 3 . Furthermore, such tests are impractical since there are no analytical solutions for relevant transient plasmas

Therefore, is very hard to separate the influence of the different processes from model uncertainties. This is especially true if both factors contribute significantly to the evolution of the plasma in a given experiment, even if it is performed under a variety of conditions (e.g. for different pressures).

However, a significant portion of the required parameters (e.g. cross-sections) should be the same for all relevant experiments. Therefore, we considered, at first, simpler experiments, which (a) include effects that seem to be important for EUV plasma dynamics, and where (b) the most of the poorly known parameters are excluded, because the corresponding effects are not present in a particular experimental setup. This route allows us to check many parts of the model and, therefore, exclude some parameters and hypotheses from consideration in the analysis of EUV induced plasma simulations.

In most experiments, the EUV power is not known accurately (e.g. within a factor of two), and the SE yield is also a poorly known parameter. But, both of these factors contribute significantly to the plasma formation. Hence, it is very interesting to consider these two contributions separately (see table 4.1).

Table 4.1: Experiments overview

\begin{tabular}{lcc}
\hline experiment & EUV & SE \\
\hline Discharge ignited by UV laser (MSU) & no & yes \\
Plasma in resonant cavity ignited by EUV (TU/e) & yes & no \\
Carbon cleaning by EUV induced plasma (ISAN) & yes & yes \\
\hline
\end{tabular}

In order to investigate how the secondary electron emission, space charge formation and electron induced ionization are represented in the model, it is interesting to begin with a pulsed hydrogen plasma without any EUV at all, Hence, we considered a pulsed hydrogen plasma that 
was ignited by an ultraviolet (UV) laser pulse. The laser pulse excited photoelectrons from one electrode, which were accelerated towards a counter electrode, generating a plasma through collisions during their transit.

This plasma has much in common with the EUV-induced plasma, e.g. space charge effects significantly influence the plasma dynamics, the plasma is transient, etc. But, the UV $(\sim 248 \mathrm{~nm})$ photons do not ionize hydrogen and produce only low energy secondary electrons. This eliminates many of the most problematic parameters, such as the EUV spectrum, EUV dose and even SE yield, because, in the model, SE production is represented as a source of low energy electrons with a time dependent intensity.

Thereby, we checked the ability of the model to (a) deal with space charge, (b) describe electron impact induced ionization of the background gas with the reasonable accuracy, and (c) describe ion drift with reasonable accuracy (because the plasma life time is determined by ion drift from the plasma to the electrodes in the presence of an externally applied electric field). Importantly, these experiments allowed the cross-sections set for electron induced processes to be chosen. These cross-sections were used for all further simulations considered in this thesis. The simulation details and their comparison with the experimental results are presented in chapter 5 .

The next step was to consider an experiment with EUV radiation, but without any secondary electrons. In the general case, even if a spectral purity filter (SPF) is used, the error margin of the EUV dose, EUV spectrum, (i.e. a poorly known function and a scalar value) must be considered. Here, we separated the EUV spectrum and EUV dose because they are typically measured by different equipment, e.g. EUV intensity is measured by the thermal gauge or EUV sensitive foil, but the EUV spectrum is typically measured by a spectrometer without absolute calibration.

Hence, an experiment with an experimental measurement of the average density of a plasma ignited by the transmission of an EUV beam through a gas volume provided an excellent way to test how direct photoionization processes are included in the model. The results of these experiments are presented in chapter 6.

Finally, we returned to the ISAN experiments with EUV radiation, where the carbon etching was observed in various conditions. We used the model to calculate the absolute fluxes and energies of different ion species, because, the model was already tested on the previous two experiments. These fluxes were then used to gain insight into the physical and chemical processes that determine the carbon etch rate. These results are presented in chapter 7. 


\title{
Chapter 5
}

\section{Plasma probe characteristics in low density hydrogen pulsed plasmas}

\begin{abstract}
Probe theories are only applicable in the regime where the probe's perturbation of the plasma can be neglected. However, it is not always possible to know, a priori, that a particular probe theory can be successfully applied, especially in low density plasmas. This is especially difficult in the case of transient, low density plasmas. Here, we applied probe diagnostics in combination with a 2D particle-in-cell model, to an experiment with a pulsed low density hydrogen plasma. The calculations took into account the full chamber geometry, including the plasma probe as an electrode in the chamber. It was found that the simulations reproduce the time evolution of the probe IV characteristics with good accuracy. The disagreement between the simulated and probe measured plasma density is attributed to the limited applicability of probe theory to measurements of low density pulsed plasmas on a similarly short time scale as investigated here. Indeed, in the case studied here, probe measurements would lead to, either a large overestimate, or underestimate of the plasma density, depending on the chosen probe theory. In contrast, the simulations of the plasma evolution and the probe characteristics do not suffer from such strict applicability limits. These studies show that probe theory cannot be justified through probe measurements. However, limiting cases of probe theories can be used to estimate upper and lower bounds on plasma densities. These theories include and neglect orbital motion, respectively, with different collisional terms leading to intermediate estimates.
\end{abstract}

\subsection{Introduction}

Low pressure $(1 . .100 \mathrm{~Pa})$ low density $\left(n_{e} \sim 10^{8} . .10^{9} / \mathrm{cm}^{3}\right)$ pulsed plasmas are commonly found in many laboratory experiments. These plasmas can exhibit complicated behavior because they can be operated in the non-local and non-stationary regime.

However, in practice, plasmas of this type are frequently investigated with the Langmuir probe technique. But the application of plasma probe diagnostics is hindered, because it strictly requires $[75,76]$ (a) that the plasma is distorted by the probe only locally in a well defined manner, and (b) that the evolution of the plasma, excluding the distorted region, is not affected by the presence of the probe.

Probe diagnostics were initially designed for glow discharge plasmas, where the applicability limits could be estimated in advance easily. Thus, Langmuir probe measurements allowed plasma parameters, such as the electron density and temperature, to be derived with the help of 
appropriate formulas. However, the probe method does not provide a technique to estimate the applicability of the method itself.

Nevertheless, in many experiments with a lower density plasma there is not enough information to justify the probe method beforehand. Thus, the plasma parameters, which are obtained from probe measurements with help of probe theory, are used to evaluate the methods applicability.

Applying a probe theory outside of its applicability limits leads to errors in the plasma parameters derived from the measured probe response. Frequently, in day to day practice, the probe method validity is estimated from two observations: (a) the probe I - V characteristic should have distinct ion and electron contributions, and (b) the plasma size should be at least a factor of 100 larger than the estimated Debye length [76]. However, these parameters, derived from the probe measurements, have a tendency to support the validity of the probe method, e.g. if the derived electron density is overestimated for a low density plasma (see section 5.4.4 for example and discussion), the second condition is satisfied in most cases.

In order to overcome these difficulties, we propose to use a combined approach, where experiments and simulations are coupled as tightly as possible, with the full experimental geometry included in simulations. In these simulations, the plasma probe is included as an additional electrode. By including the probe in the model, any effect that it has on the plasma is naturally included in the dynamics. This approach does not have any of the restrictions discussed above.

In general this requires a 3D plasma model. Such simulations are extremely time consuming, but, fortunately, it is possible to reduce the model dimensions by using of an axis-symmetrical configuration for the experimental setup. This allows a $2 \mathrm{D}$ cylindrically symmetric model to be used for the plasma simulation, which is much faster than a 3D model.

In this paper, we show that, especially in the case where the spatial homogeneity of the plasma cannot be easily verified, one needs to be very careful interpreting the results from probes. We consider a discharge that produces probe characteristics with the proper response. Using several probe theories (see below), we show that they all self consistently report densities, temperatures, and dimensions that would lead one to conclude that the probe theory is correct. However, not only are these theories mutually inconsistent, a 2D PIC model of the same plasma shows that the probe theories are highly likely to be inconsistent with the experimental plasma densities as well. The difference between the PIC model and the probe theories was attributed to the distortion of the plasma by the probe, and to transient plasma dynamics.

\subsection{Experimental setup}

The interior configuration of the vacuum chamber, in which the low density $\mathrm{H}_{2}$ pulsed plasma was generated, is presented in Fig. 5.1. The chamber was pumped with a turbomolecular pump to keep the residual gas pressure lower than $0.003 \mathrm{~Pa}$. During experiments, hydrogen flowed through the chamber at several sccm, controlled by a gas flow meter. The hydrogen pressure in the chamber was varied in the range of 0-60 $\mathrm{Pa}$.

The aluminum electrodes are circular plates with a $10 \mathrm{~cm}$ diameter. The distance between the electrodes can be varied in the range of $2-5 \mathrm{~cm}$. In the experiments, the bottom electrode was negatively biased at $-200-0 \mathrm{~V}$, while the top electrode was grounded. The bias was kept at a 


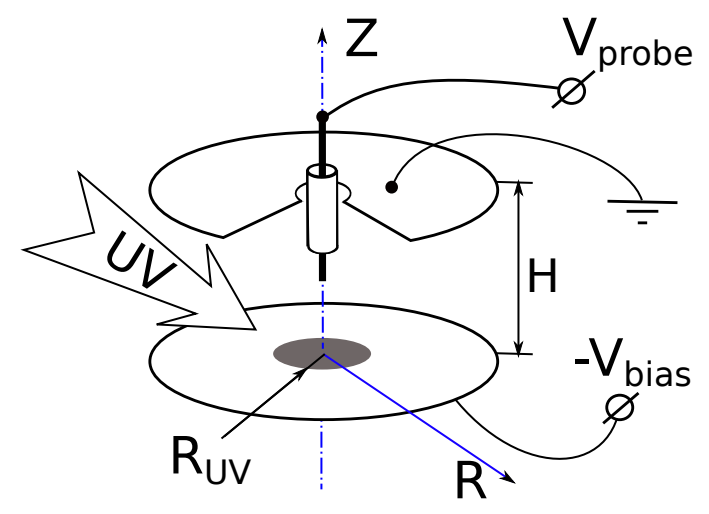

Figure 5.1: Configuration of the experimental chamber. The electrodes are $10 \mathrm{~cm}$ in diameter and separated by $5 \mathrm{~cm}$. The plasma probe passes through the center of the top electrode and is $3 \mathrm{~cm}$ from the bottom electrode. The open end of the probe is $1 \mathrm{~cm}$ in length and $0.5 \mathrm{~mm}$ in diameter. The remainder of the probe is covered by a dielectric with a length of $1 \mathrm{~cm}$ and a diameter of $1.3 \mathrm{~mm}$. The plate holders and chamber walls are not show, because the vacuum chamber is large (cylinder $20 \mathrm{~cm}$ in diameter and $20 \mathrm{~cm}$ in height).

constant value during the plasma ignition and decay. The plasma was ignited by the photoelectrons emitted from the bottom electrode during the UV laser pulse. Most of these electrons are reflected back to the bottom electrode by the space charge field. The electrons which pass the potential barrier, are accelerated by the applied bias field and ionize the background gas, thus igniting the plasma.

A KrF excimer laser (LPX210) was used as the source of the UV radiation that produces photoelectrons at the bottom electrode. The temporal profile of the laser pulse consists of a short pulse with a full width half maximum (FWHM) of $<50 \mathrm{~ns}$ and a tail, extending up to 200-300 ns in duration (see Fig. 5.2). The pulse energy, incident on the bottom electrode, was approximately $10 \mathrm{~mJ} /$ pulse, which was only $20 \%$ of total laser pulse energy. Approximately the same fraction of the total laser energy was reflected to a detector by a beam splitter to control the laser pulse energy during the experiment. The laser beam was passed through a diaphragm to obtain a uniform intensity distribution with a diameter of $1.3 \mathrm{~cm}$ at the bottom electrode. The laser light was incident at an angle to the electrode after entering the chamber through a quartz window.

The plasma probe was installed along the symmetry axis of the chamber. The probe was $0.05 \mathrm{~cm}$ in diameter and $1 \mathrm{~cm}$ long, the glass shield that covers the rest of the probe was $0.13 \mathrm{~cm}$ in diameter. The probe measurements were automated with a NI-DAQ card and specially developed signal processing unit (SPU), consisting of a trans-impedance isolated amplifier, differential amplifier, and probe bias control. This unit allows probe currents down to $10 \mathrm{nA}$ to be measured in the band of approximately $6 \mathrm{MHz}$. The signals amplified by the SPU were transferred to an oscilloscope (Tektronix TDS3032). The overall control of measurements and the experimental setup was performed with a computer program via the NI-DAQ card, as well as GPIB, and RS232 interfaces. The obtained data were stored on a server for further analysis. 


\subsection{Model}

The plasma was modelled using a two-dimensional ( $r z$ cylindrically symmetric) particle-in-cell (PIC) model. The plasma species include electrons, $\mathrm{H}^{+}, \mathrm{H}_{2}^{+}$and $\mathrm{H}_{3}^{+}$as particles for accurate description of processes in the plasma sheath. Our model follows the general PIC scheme, described elsewhere [28].

The combination of measurements and simulations of discharge characteristics and plasma probe measurements allows the number of free parameters to be reduced. These parameters (e.g. ion induced electron emission yield, photo electron yield, initial electron energy distribution etc.) are required to perform simulations, but the range of values found in literature are typically too large for accurate modeling. It is worth noting here that many such parameters are intrinsically setup dependent (i.e. the photo electron yield could be very sensitive to the surface conditions). Thus, preferably, such parameters should be measured.

An accurate measurement of all unknown parameters is often unfeasible. To solve this issue, the model was developed in parallel to the experimental setup. Thus, many of the unknown parameters were obtained with reasonable accuracy using the results from simple experiments, which were performed as a test procedure during the construction and calibration of the experiment.

\section{UV laser and electron spectrum used as input to the model}

In the experiment, an excimer laser, operating at a wavelength of $248 \mathrm{~nm}$, generates photoelectrons from the bottom electrode. In the model, this process is included as a photoelectron source on the bottom electrode. On each time step, when the laser is active, photoelectrons are released from the irradiated part of the bottom electrode to the computational domain. The amount of injected electrons is linearly proportional to the instantaneous laser intensity. The electron energies are sampled according to a distribution, as discussed below. The velocities of the injected electrons are distributed according to a cosine law relative to the normal direction to the boundary.

The initial electron energy distribution and the temporal profile of the laser pulse are important parameters that significantly influence the plasma that is formed later. Under vacuum conditions, where no plasma is ignited, the temporal profile of the current contains information about the laser pulse shape and the effective photoelectron yield. We used this experiment and the following physically motivated choice of parameters to define the effective electron source for the model.

The laser energy per pulse in the experiment was $10 \mathrm{~mJ}$. The typical photoelectron emission yield is estimated to be $10^{-4}-10^{-3}$ electrons/photon [25], thus, the maximum collected charge is approximately $20-200 \mathrm{nC}$. However, in the experiment, the collected charge per pulse under vacuum conditions was approximately $0.2 \mathrm{nC}$. Hence, the space charge current limiting was very high during the main part of the laser pulse.

The space charge potential barrier formation leads to a locally high electron density. The electrons, which are trapped by the barrier, have small energies, thus, Coulomb collision processes should dominate. From a computational point of view it is not reasonable to compute the full collision kinetics of the cold electrons, since it is known to lead to the formation of a 
Maxwellian tail in the energy distribution, and the detailed kinetics depend on the exact initial distribution and photoelectron yield, which are not known. Thus, a Maxwellian function was taken as the initial electron energy distribution function (EEDF).

The effective temperature for this EEDF was heuristically chosen from two observations. Firstly, the bottom electrode was made from aluminum, which might by partially oxidized. A number of values for the work function of this material have been reported, the lowest of which is about $4 \mathrm{eV}$ [40]. Secondly, since the photoelectron yield is not known precisely, we only need to find a reasonable combination of effective temperature and yield to reproduce the chargebias characteristic, as measured experimentally under vacuum conditions. Thus, the effective temperature for EEDF was chosen to be $0.5 \mathrm{eV}$, and the value for photoelectron yield was finetuned from the measured vacuum charge bias characteristics of the system. Because of the significant space charge screening, the sensitivity for the particular choice is small.

A scan over laser pulse energies from $3 \mathrm{~mJ}$ to $15 \mathrm{~mJ}$ was performed to ensure that the experiment was stable. It was found that the vacuum discharge characteristics have the lowest sensitivity to the laser power variation near $10 \mathrm{~mJ} /$ pulse. However, while the integral value was stable, there were signs of higher order processes (e.g. two photon photoelectric effect), since the value of the vacuum current peak changed near a laser pulse energy of $6 \mathrm{~mJ}$ (see Fig. 5.2). These experiments were included into the fitting procedure, and it was found that while for all energies below $6 \mathrm{~mJ} /$ pulse the above described procedure produced consistent results, it was not possible to reproduce the current peak value for $10 \mathrm{~mJ} /$ pulse and above due to space charge screening. For this reason, a very small amount of $4 \mathrm{eV}$ electrons, with a yield of approximately $10^{-8}$, was added to the electron source to describe the case of $10 \mathrm{~mJ} /$ pulse laser energy.

As mentioned above, the laser pulse shape has a tail (up to $300 \mathrm{~ns}$ ) in comparison to the $60 \mathrm{~ns}$ main pulse (See Fig. 5.2). This tail plays a very important role in plasma formation as discussed in section 5.4.1. Unfortunately, the radiation intensity in the laser tail was too low to accurately measure with our equipment. This fact also suggests that one should not expect a space charge effect. For this reason, the EEDF for the electrons generated by the tail of the pulse is constant for all electron energies less than $1 \mathrm{eV}$, and zero for higher energies.

A fitting procedure was used to derive the laser tail shape from the measured discharge characteristics in vacuum. During this procedure the pulse shape was represented as the sum of the measured laser pulse and a tail. The electron yield was chosen such that it reproduced the peak current value and the tail current simultaneously.

\section{Ion induced electron emission}

It is important to take into account ion induced electron emission, since free electrons are accelerated by the electric field to the top electrode and produce additional electrons and ions through impact ionization. The electrons produced through impact ionization contribute significantly to the later dynamics of the plasma and should be taken into account.

The bottom electrode in the experiment was biased at $-200-0 \mathrm{~V}$, therefore, the maximum ion energy is about $200 \mathrm{eV}$. For this energy range, the ion induced electron emission can be represented as a sum of potential emission (PE) and kinetic-induced emission (KE) [68, 71]. The $\mathrm{PE}$ yield is determined by the combination of the ion and target material, however, for the slow ions it is independent of the ion velocity. But, the KE yield (see below) strongly depends on the 
ion velocity.

In the model, the PE probability to emit an electron per one ion impact at the bottom electrode is computed with the following semi-empirical formula from [67]

$$
\gamma \approx \frac{0.2}{E_{F}}\left(0.8 E_{i}-2 W\right)
$$

Here $E_{F}$ is the metal Fermi energy, $E_{i}$ is ionization energy of the ion and $W$ is the metal's work function. The applicability range of Eqn. 5.1 is $3 W \leq E_{i} \leq\left(2 E_{F}+W\right)$, the accuracy of this approximation in these range is not better than 10\%. Outside this range, the accuracy of Eqn. 5.1 slowly decreases.

The top and bottom electrodes (see Fig. 5.1) are made from aluminum. The tabulated values for aluminum are $E_{F}=11.7 \mathrm{eV}, W=3.8-4.5 \mathrm{eV}$. The large spread in work function can be attributed to a combination of the effect of the surface crystalline structure [40] and of surface oxide, which can lead either to an increase or a decrease of the observed work function, depending on the exact conditions [77]. The plasma probe is made from molybdenum, which has a Fermi energy and work function of $E_{F}=6.8 \mathrm{eV}, W=4.2-4.6 \mathrm{eV}[40,78]$.

For a hydrogen plasma there are three types of ions possible. The ions have ionization energies of: $\mathrm{H}^{+} 13.6 \mathrm{eV}, \mathrm{H}_{2}^{+} 15.4 \mathrm{eV}$, and $\mathrm{H}_{3}^{+} 3.6 \mathrm{eV}$. The value used for $\mathrm{H}_{3}^{+}$here is the ionization potential for a long lived $\mathrm{H}_{3}$ taken from [79]. This definition of $\mathrm{H}_{3}^{+}$ionization energy is less than twice the energy of the work functions of aluminum or molybdenum, thus, $\mathrm{H}_{3}^{+}$does not produce any potential emission. Hence, the $\mathrm{PE}$ probability for $\mathrm{H}^{+}$is approximately $0.04 \pm 0.02$ on aluminum and $0.06 \pm 0.02$ on molybdenum, while for $\mathrm{H}_{2}^{+}-0.07 \pm 0.02$ on aluminum and $0.1 \pm 0.02$ on molybdenum. In these estimates we included an error of at least $10 \%$ due to the approximation used and further $10 \%$ error due to the poor knowledge of work function.

There are complicated KE models [69], but they require some fitting parameters to be defined. Since the exact surface conditions are not known well, the same accuracy is obtained by simply fitting to experimental data [71]. Therefore, we follow the same approach as in [70], and use a fit to the experimental data [71] to determine for KE for all metallic surfaces.

$$
\gamma_{K E} \approx 6.2 \cdot 10^{-5} \cdot(E[\mathrm{eV} / \mathrm{amu}])^{1.15}
$$

Here $E$ is the kinetic energy of the ion per ion mass.

The physical motivation for this approach is as follows. For many metals and conductive materials, the work function $(W)$ is approximately $5 \mathrm{eV}$ and the quasi-classical threshold of KE is $v_{i}>W /\left(2 k_{F}\right)$, here $v_{i}$ is the ion velocity and $k_{F}$ is a Fermi impulse. KE depends strongly on the ion type and target material, especially for heavy ions. But, in experiments [71], the KE probability, as a function of velocity, varies only by a factor of two or less for $\mathrm{H}^{+}, \mathrm{H}_{2}^{+}$and $\mathrm{H}_{3}^{+}$ (for a gold target). Similar experiments indicate that, for a given ion species, KE is similar for a broader range of target materials [72].

In our simulation, the use of an ion-type-dependent ion-induced electron emission coefficient leads to time-dependent ion-induced emission, because, just after the UV pulse, the main ion is $\mathrm{H}_{2}^{+}$and the ion induced emission coefficient is large. However, due to a very efficient ion conversion reaction $\mathrm{H}_{2}^{+}+\mathrm{H}_{2} \rightarrow \mathrm{H}_{3}^{+}+\mathrm{H}$, the role of ion-induced emission significantly decreases after a characteristic ion conversion time. 
Although the approximations for $\mathrm{PE}$ and $\mathrm{KE}$ are rather simple, they provide reasonable estimates of ion-induced emission. For instance, this simple model allows us to distinguish between a discharge initiated by a laser pulse without a tail and a large ion-induced emission coefficient, and the case with a smaller ion-induced emission coefficient and a laser pulse with a tail (as in Fig. 5.3).

\section{length scales, grid resolution}

The spatial resolution of the rectilinear grid is chosen to resolve two main characteristic length scales: the space charge potential well and the Debye length. The space charge potential well length scale can be estimated from the analytical solution for a 1D diode with a Maxwellian initial electron energy distribution [74]. In the considered case, most photoelectrons are reflected back to the surface. Thus, it is possible to simplify the formulas from [74] and obtain

$$
z_{m} \simeq 0.1 \mathrm{~cm} \times \frac{(T[\mathrm{eV}])^{3 / 4}}{\left(I\left[\mathrm{~mA} / \mathrm{cm}^{2}\right]\right)^{1 / 2}}
$$

Here, $T$ is the initial temperature of emitted photoelectrons, $I$ is the current density near the cathode and $z_{m}$ is the distance from the cathode and the bottom of the space charge potential well. For $T \sim 0.5 \mathrm{eV}$ and $I \sim 2 \mathrm{~mA} / \mathrm{cm}^{2}$, one obtains $z_{m} \sim 0.04 \mathrm{~cm}$. Since the UV spot diameter is much larger than the estimated $z_{m}$, the $z_{m}$ length scale should only be resolved in the $z$ direction near the bottom electrode. For the plasma region, the grid cell size is estimated as the Debye radius, $r_{D}$, for a plasma with $n_{e} \sim 10^{9} / \mathrm{cm}^{3}$, and $T_{e} \sim 0.5 \mathrm{eV}$, which corresponds to $r_{D} \sim$ $0.02 \mathrm{~cm}$. We ran several tests to ensure that the grid resolution does not affect our results.

The displacement currents to the probe and electrodes are also included in the model. They are computed at each time step as the numerical derivative of the electric field over each of the electrode boundaries. Inclusion of the displacement current allows direct comparison between the simulated and experimental discharge currents.

To calculate the production of the different ion species, a set of the cross-sections for the hydrogen chemistry was assembled from the several sources. The data from [48] was used as a base, and extended using the work of [62], [64].

The procedure described in [42] is used to perform Monte Carlo collisions with the background gas. This procedure allows a reaction channel to be chosen before sampling the reaction probability, which offers significant performance improvement compared to the Null collision scheme [41].

We neglect the formation of $\mathrm{H}^{-}$. The cross-section of dissociative electron attachment is significant only if $\mathrm{H}_{2}$ is high vibrationally excited (e.g. $v=4$ ) [44]. However, the density of $\mathrm{H}_{2}$ molecules in these states is expected to be very low, since the corresponding cross-sections of the direct excitations are small [59]. The stepwise excitations are expected to give negligible contribution due to small ratio of the concentrations of vibrationally excited $\mathrm{H}_{2}$ and $\mathrm{H}_{2}$ in the ground state.

We tested the consistency of our implementation by modeling swarm experiments and found good agreement with experimental values [54] for electron ionization coefficients, electron mobility and for $\mathrm{H}^{+}, \mathrm{H}_{3}^{+}$mobility in hydrogen [55]. 


\subsection{Results and discussion}

\subsection{1 discharge characteristics}

Let us begin with the analysis of the discharge characteristics of the experimental setup without a plasma probe.

During the laser pulse, only a small amount of photoelectrons enter the chamber because of the space-charge-induced potential barrier. This barrier is not just due to the electron cloud trapped near the bottom electrode, but is built up by all the excess negative charge present in the chamber. Hence, simulations under vacuum conditions (i.e., no plasma) provide a good estimate of the temporal profile of the laser pulse, but they are not very sensitive to the magnitude of electron emission. In the model, the temporal profile of the laser pulse was fine-tuned such that it reproduced the current maximum for the case of $30 \mathrm{~Pa},-200 \mathrm{~V}$ bias. This was sufficient to yield good agreement with experimental data for the maximum current under all other considered conditions.

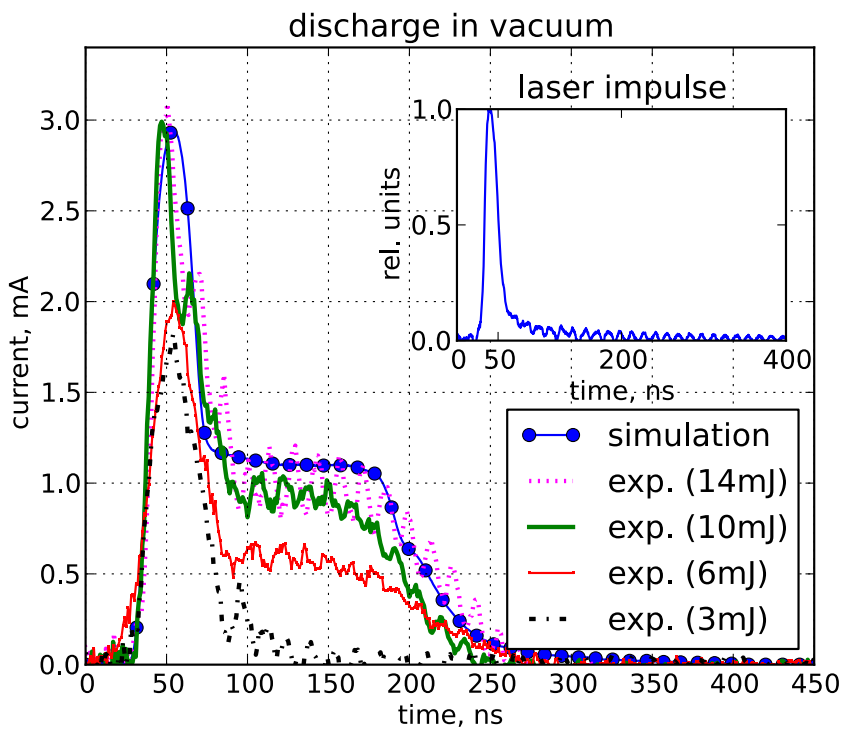

Figure 5.2: Discharge current in vacuum conditions, -200V bias. Laser pulse temporal profile for $10 \mathrm{~mJ} /$ pulse (inset). The current due to the main laser pulse is in the saturation regime. The difference between $6 \mathrm{~mJ} /$ pulse and $10 \mathrm{~mJ} /$ pulse peak currents can be attributed to some higher order effects, since the peak values for $3 \mathrm{~mJ} /$ pulse and $6 \mathrm{~mJ} /$ pulse are very close. Step-like current tail is due to laser tail. The current tails for $10 \mathrm{~mJ} /$ pulse and $14 \mathrm{~mJ} /$ pulse are very close in magnitude, justifying the choice of $10 \mathrm{~mJ} /$ pulse as the energy for experiments. The simulation data correspond to the fine tuned laser pulse shape (see text) for $10 \mathrm{~mJ} /$ pulse. The temporal pulse shape of the laser, as measured by the detector is presented in the insert.

The current as function of time for $-200 \mathrm{~V}$ bias at different background pressures is presented in Fig. 5.3. The laser pulse tail (see Fig. 5.2) produces a significant contribution to the plasma formation (see comparison of cases with and without tail in Fig. 5.3). This happens because 


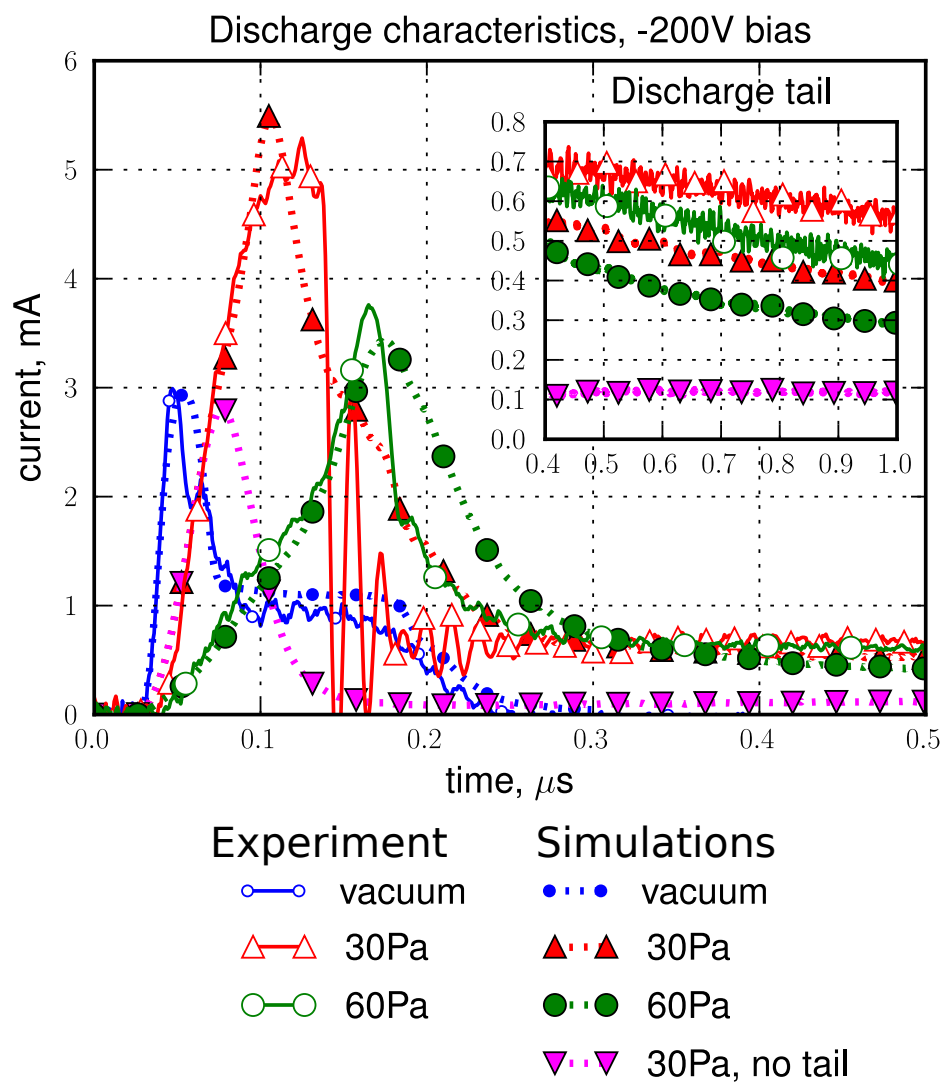

Figure 5.3: Discharge current as function of time for various hydrogen pressures. The tail of discharge is shown in inset in the same units as main plot. Note, that the laser pulse tail (see Fig. 5.2) produces a significant contribution to plasma formation, as suggested by comparison of simulation results for $30 \mathrm{~Pa}$ with and without laser pulse tail.

the electrons produced during the body of the laser pulse provide ionization in the chamber, which leads to the formation of the plasma. The presence of this plasma changes the potential distribution inside the chamber, leading to an increased field strength near the bottom electrode, which, in turn, increases the efficiency of ionization, thus, enhancing the effect of the laser pulse tail.

Despite reproducing both the slope of the current increase, and the current maximums for both pressures, the magnitude of the simulated discharge tail is about $30 \%$ lower than in the experiment. Agreement between our simulations of the discharge tail, and the experimental data can be obtained by varying the tail of the laser pulse. Under these conditions, however, the peak position and width of the current were significantly distorted at pressures of $30 \mathrm{~Pa}$ and $60 \mathrm{~Pa}$. Alternatively, increasing $\mathrm{PE}$ and $\mathrm{KE}$ also yields a discharge tail in agreement with experimental data. This, however, results in unrealistically high ion induced electron emission yield for $\mathrm{H}_{3}^{+}$ion (e.g. $\sim 0.05$ electron/ion on average). Moreover, the model fails to reproduce the Langmuir probe measurements in this case. 
Therefore, the disagreement between the model and experimental data is probably due to an accumulation of minor differences between the input parameters of the model and the experiment. These could be, for example, the laser spot size, uncertainties in the cross-sections set, pulse-to-pulse variations of laser pulse tail, etc.. Nevertheless, the $30 \%$ difference between the plasma model and the experimental data is reasonably accurate for the probe simulations.

\subsection{2 plasma scalability}

Additional experiments were performed to study the plasma scalability for different distances between electrodes, with fixed $p d$ parameter (here is $p-$ hydrogen pressure, $d$ is the distance between electrodes) and fixed reduced electric field strength $E / p=U_{\text {bias }} /(p d)$. The integral of the discharge current for different distances between the electrodes is presented in Fig. 5.4. The obtained plasma did not scale with $p d$ parameter. It showed that dynamic effects were important in plasma generation.

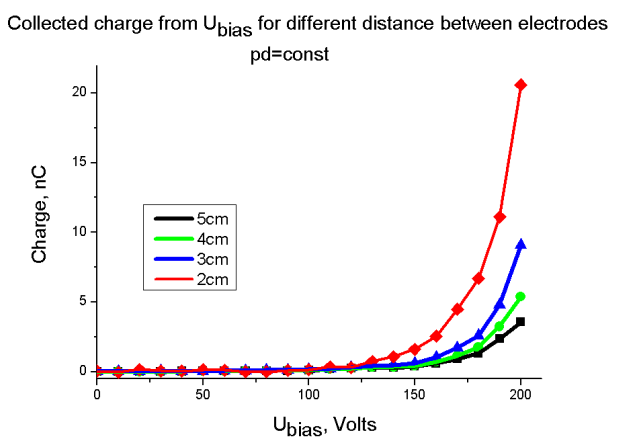

Figure 5.4: Collected charge for different distances between electrodes, but constant $p d$ (for $d=5 \mathrm{~cm}, p=30 \mathrm{~Pa})$.

\subsubsection{Axially symmetric plasma probe}

To make a detailed investigation of plasma evolution the plasma probe diagnostic was used. The probe was installed on the symmetry axis of the chamber to allow direct simulations of the probe characteristics with the 2D plasma model.

The probe measurements were performed at $30 \mathrm{~Pa}$ and $60 \mathrm{~Pa}$ hydrogen, the bias between the electrodes was $-200 \mathrm{~V}$ in both cases. The simulated and measured probe characteristics were shifted by $1 \mathrm{~V}$ for $30 \mathrm{~Pa}$ and by $2 \mathrm{~V}$ for $60 \mathrm{~Pa}$. This shift could have numerous causes, therefore, the measured probe characteristics were shifted by these values to the negative probe voltages. The measured probe characteristics and the comparison with the simulations are presented in Fig. 5.5 and Fig. 5.6. All presented simulations use the same initial input data apart from those varied in the experiments.

To take into account the characteristic response time of the SPU, the simulated probe response was numerically convoluted with $C \cdot \exp \left(-t / t_{0}\right)$, here $t_{0} \sim 100 \mathrm{~ns}$ is the SPU response 
time and constant $C$ is chosen such that the collected charge is preserved during numerical integration.

The agreement between the simulated and measured probe characteristic is good up to $2.5 \mu \mathrm{s}$ after the start of the UV pulse for the both pressures. At later times, the agreement is worse, due to the response of the simulated probe changing because the tip of the probe is no longer in the region containing a plasma (see Fig. 5.8). The transition of the probe from inside to outside the plasma (for the case of $30 \mathrm{~Pa}$ ) occurs between $2.5 \mu \mathrm{s}$ and $3.5 \mu \mathrm{s}$, however, the exact transition time is very sensitive to the model input parameters. After the transition (4.0-4.5 $\mu \mathrm{s}, 30 \mathrm{~Pa}$, $200 \mathrm{~V}$ ), the good agreement between the measured and modeled ion contribution to the probe characteristic is restored. Furthermore, despite the plasma no longer covering the entire probe, the probe response appears normal.

For the case of $60 \mathrm{~Pa},-200 \mathrm{~V}$ (Fig. 5.6), the transition occurs later due to a larger contribution from collisions with the background gas. Therefore, the ion part of the probe characteristics for $4.5 \mu \mathrm{s}$ for $60 \mathrm{~Pa}$ slightly deviates from the experimental one, while for $30 \mathrm{~Pa}$ the agreement is good.

The electron contribution to the simulated probe characteristics in Fig. 5.5 deviates significantly from the experiment occasionally from $2 \mu$ s onwards for a pressure of $30 \mathrm{~Pa}$. But, at $60 \mathrm{~Pa}$ (Fig. 5.6), the deviation of the electron part is much smaller and only pronounced from $3.5 \mu \mathrm{s}$ and for a probe bias larger than $5 \mathrm{~V}$. These differences can be attributed to the combined effects of the uncertainties in electron - neutral cross-section set and some secondary effects (e.g. reflection of electrons from the aluminium surface), which are not taken into account. However, the simulations accurately reproduce the transition from the ion part to the electron part of the probe characteristic in both cases.

Interestingly, the apparent shift of the probe characteristic in Fig. 5.6 with time is reproduced by the model. Usually such a shift in the stationary plasma conditions may be explained by partial presence of the probe in or near the plasma sheath. However the reason of the shift in the case of a pulsed plasma may have other causes. As is seen in Fig. 5.9, the change of the sign of the probe current can not be explained by the presence of the probe in the sheath.

However, the shape of simulated plasma distortions depends on the dimensions on the probe. We simulated the plasma evolution with ten times thinner and two times shorter probe. We found that, although the distortion of the plasma around the probe was different, it was still significantly distorted. The shape of distortion was similar to described in [80].

Interestingly, for the ten times thinner probe the role of ion neutral collisions are expected to be of the same importance for the analysis of the probe response as for the used probe. The size of plasma sheath near the probe is determined by the the Debye length of the plasma. For the considered conditions, it can be estimated as $\lambda_{D} \sim 0.23 \mathrm{~mm}$ (i.e. for $n_{e} \sim 5 \cdot 10^{8} 1 / \mathrm{cm}^{3}$ and $T_{e} \sim 0.5 \mathrm{eV}$ ). Therefore, we do not expect the sheath size to decrease significantly for a probe with a radius of $0.025 \mathrm{~mm}$ as compared to $0.25 \mathrm{~mm}$ for the same probe biases. Hence, the number of the collisions in the probe sheath should be comparable in both situations.

Therefore, the application of a smaller probe in the considered discharge does not necessarily lead to negligible distortion nor to transition from collisional to collision less ion currents to the probe. 


\subsubsection{Comparison between computed plasma density and that derived from measured probe characteristic}

The 2D particle-in-cell simulations showed that probe diagnostics are not well suited to the considered plasma, because the plasma is spatially inhomogeneous along the probe length (see Fig. 5.8 with probe and Fig. 5.7 without probe). However, it is still interesting to apply probe theories to the measured probe characteristics, since in the general case, the distortion of the plasma by the probe is not known in advance. Furthermore, the probe response appears to be normal with distinct ion and electron parts, which would normally be used as evidence that probe theory can be applied.

We applied the Bernstein-Rabinowitz-Laframboise (BRL) [81], Allen-Boyd-Reynolds (ABR) $[82,83]$ and Talbot and Chou theory [84] modified by Tichý et al. (TCT) [85] to analyze the probe I - V characteristics. We used the ESP_BRL and ESP_ABR programs [76] to obtain BRL and ABR fits for the probe characteristics. The fit results are presented in Table.5.1. The electron temperature in the simulations after $1 \mu \mathrm{s}$ was approximately $0.3 \mathrm{eV}$ for $60 \mathrm{~Pa}$ and $\sim 0.5 \mathrm{eV}$ for $30 \mathrm{~Pa}$. This corresponds to the estimate from the probe response, but the simulated EDF has a non-Maxwellian tail due to the presence of an external bias.

The most abundant ion in the considered conditions is $\mathrm{H}_{3}^{+}$[62]. The mobility of this ion in $\mathrm{H}_{2}$ increases with increasing ion energy, due to a local minimum in the effective momentum transfer cross-section around $0.8 \mathrm{eV}$ [62] in the center of mass reference frame. The energy range, where mobility changes significantly is smaller than the absolute value of the probe voltage. Modification of the probe theory to include variable collisional cross-sections is out of the scope of this paper, since this effect is included in the PIC model. Therefore, we performed the fitting procedure using the TCT theory with a collisional correction corresponding to the zero field mobility of $\mathrm{H}_{3}^{+}$in $\mathrm{H}_{2}$, and for increased mobility, the corresponding reduced mobilities are $\sim 11.3 \mathrm{~cm}^{2} /(\mathrm{V} \cdot \mathrm{s})$ and $\sim 16 \mathrm{~cm}^{2} /(\mathrm{V} \cdot \mathrm{s})$. The resulting ion densities obtained are presented in the Table 5.1.

It is also worth noting that an a posteriori estimate of the applicability of the probe method can lead one to incorrectly assume that a chosen probe theory is appropriate. Consider that, in the cylindrically symmetric setup here, the plasma density and radius can be estimated from the probe response via the BRL and TCT theories. The radial dimension of the plasma can be estimated to be $r \geqslant \sqrt{Q_{c} /\left(\pi q_{e} N_{i} d\right)}=1.5 \mathrm{~cm}$, where $Q_{i} \sim 4 \mathrm{nC}$ (see Fig. 5.4), $d=5 \mathrm{~cm}$ is the distance between the electrodes, and $N_{i}$ is the density estimate obtained from the chosen probe theory. Therefore, we conclude (incorrectly) that the ratio of radial plasma size to the Debye radius is, in this case, at least 75 , based on an estimated plasma density of $7 \times 10^{8} 1 / \mathrm{cm}^{3}$ from probe theory. From the arguments above, it would be argued that the probe theory will provide reasonable estimates of the plasma density. However, this conclusion is wrong, since the simulations show that the plasma density varies significantly near the probe.

The differences between predictions of the different theories are large (see Table 5.1). The BRL results correspond to the upper estimate of the plasma density, while ABR provides the lowest estimate for all probe biases. The results obtained with help of TCT theory tends to provide intermediate results. Similar trends are commonly reported elsewhere (for example in [86, 87]).

Nevertheless, as reported in $[86,87,88]$, the plasma density tends to be between the results of BRL and ABR in many different experiments. Furthermore, the plasma density estimates 
Table 5.1: Comparison of the ion density obtained from the simulations to the calculated with help of the different probe theories from the measured probe response

\begin{tabular}{|c|c|c|c|c|c|c|c|}
\hline & \multirow{3}{*}{$\begin{array}{c}\operatorname{time}^{a)} \\
\quad \mu \mathrm{s}\end{array}$} & \multirow{3}{*}{$\begin{array}{l}\mathrm{T}_{e} \\
\mathrm{eV}\end{array}$} & BRL & $\mathrm{ABR}$ & $\left.\mathrm{TCT}^{b}\right)$ & \multicolumn{2}{|c|}{ simulation } \\
\hline & & & [81] & {$[82,83]$} & [85] & with probe ${ }^{c)}$ & no probe $e^{d)}$ \\
\hline & & & \multicolumn{5}{|c|}{ plasma density $10^{8} / \mathrm{cm}^{3}$} \\
\hline \multirow{3}{*}{ 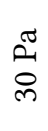 } & 1 & 0.5 & 7.5 & 1.4 & $6.5-9$ & $1-5$ & $3-5$ \\
\hline & 2 & 0.3 & 5 & 0.6 & $3-5$ & $1-5$ & $2.5-4.5$ \\
\hline & 3 & 0.3 & 3 & 0.5 & $1.2-2$ & $0.5-5$ & $2-4.5$ \\
\hline \multirow{3}{*}{$\begin{array}{l}\sigma \\
2 \\
0\end{array}$} & 1 & 0.4 & 6 & 1 & $4-7$ & $1-5$ & $1-2.5$ \\
\hline & 2 & 0.3 & 3.5 & 0.4 & $1-2.5$ & $1-5$ & $1.5-2.5$ \\
\hline & 3 & 0.3 & 2 & 0.3 & $0.7-1.0$ & $0.5-5$ & $1.5-2.5$ \\
\hline
\end{tabular}

a) Time is relative to the start of the UV pulse.

b) The lower estimate of the ion density corresponds to the lower estimate of the reduced mobility of $\mathrm{H}_{3}^{+}$in $\mathrm{H}_{2}$ (see text).

c) The simulated plasma density near the probe significantly depends on the probe bias. The lower limit corresponds to the spatial minimum of the plasma density for a bias of $-32 \mathrm{~V}$ on the probe, the upper limit corresponds to the spatial maximum plasma density for $0 \mathrm{~V}$ on the probe. Both minimum and maximum values are estimated in the quasi neutral region of the plasma.

$d$ ) The plasma density is sampled over the region corresponding to probe position in experiments and simulations with probe.

from many collisional probe theories are also bounded by the density estimates of BRL and ABR. Hence, it is reasonable to take the difference between the density estimates of BRL and $A B R$ as an approximation of the error margin of the probe measurements if the validity of the probe theory for the case of the plasma in question has not been validated by some independent numerical or experimental technique.

The present study shows, that in spite of the plasma's significant spatial inhomogeneity, the probe can still have the expected response with distinct ion and electron parts. The simulations showed that, even when the probe tip is outside of the plasma, the probe response varies smoothly in time (see Fig. 5.8). These significant distortions can not be easily deduced from the probe response alone.

Therefore, an independent method for evaluating the applicability of the probe method should be used, e.g. optical emission spectroscopy. If independent diagnostics can not be applied, we recommend to estimate the uncertainty as the difference between the fit results of BRL and ABR.

\subsection{Conclusions}

In this paper, we have reported the results of a comparison between the predictions of a 2D PIC model, and experimental measurements of a low density transient plasma.

Our 2D PIC model of the plasma evolution takes into account the full geometry of the chamber. Therefore, it allows other experimental data, such as discharge characteristic measurements, 
to be used to verify assumptions about unknown and/or poorly known parameters, and to check the applicability of the model itself on the integral parameters before analysis of the complicated plasma probe characteristics.

Our results show good agreement between time resolved simulated and measured probe characteristics for two pressures with the same parameter set. This shows that the simulated plasma parameters, such as electron and ion densities, can be relied upon, because, in our simulations, the probe was included as an electrode. Therefore, the interaction of the probe with the plasma is taken into account directly.

Our measurements also show that the measured probe characteristics have the proper shape with distinct ion and electron contributions, which would normally be taken as a sign that the parameters estimated from probe measurements would be accurate.

Nevertheless, probe theories, in the case of a low density non-stationary plasma, should be used with caution. Because, our simulation results demonstrate that, even in the case where the distortion of the plasma by the probe is obviously large (see Fig. 5.8), the probe response appears to be normal. Therefore, the application of the probe theory may yields misleading results, which in our case, is the overestimation of the plasma density by a factor of three to seven along the surface of the probe for the BRL and TCT theories and significant underestimation of the maximum plasma density by the ABR theory.

Hence, in the general case, the application of probe theory cannot be justified by probe measurements alone, and an other method should be used to verify the results of the probe method. If independent diagnostics can not be applied, we recommend to estimate the uncertainty as the difference between the fit results of BRL and ABR theories.

\section{Acknowledgments}

The authors would like to thank Tatiana Rakhimova for helpful discussion. This work is part of the research program "Controlling photon and plasma induced processes at EUV optical surfaces (CP3E)" of the "Stichting voor Fundamenteel Onderzoek der Materie (FOM)" which is financially supported by the Nederlandse Organisatie voor Wetenschappelijk Onderzoek (NWO). The CP3E programme is cofinanced by Carl Zeiss SMT GmbH (Oberkochen), ASML (Veldhoven), and the AgentschapNL through the Catrene EXEPT program. 
30Pa, -200V bias

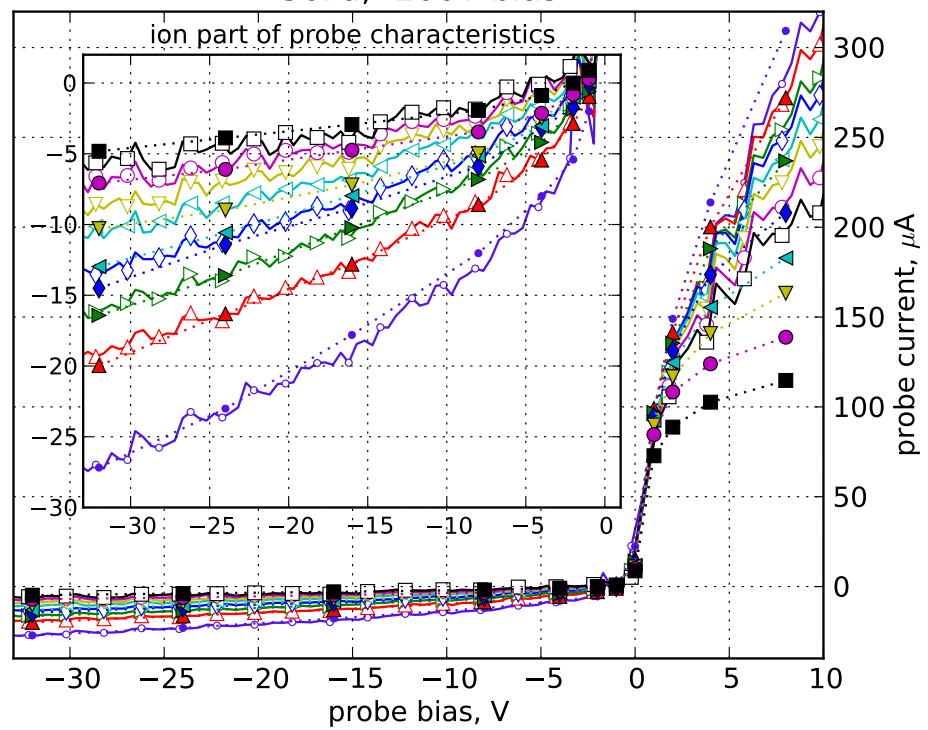

Experiment

\begin{tabular}{|c|c|}
\hline 01 & $\triangleleft \triangleleft 3$ \\
\hline+ & $\nabla \longmapsto 3$ \\
\hline & -0 \\
\hline & \\
\hline
\end{tabular}

Simulations

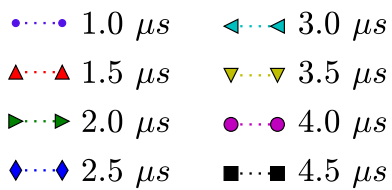

Figure 5.5: Comparison between simulated and measured probe characteristic for $30 \mathrm{~Pa}$ and $-200 \mathrm{~V}$ bias. The ion part of the probe (scales: $\mu \mathrm{A}$ vs $\mathrm{V}$ ) characteristic is shown in the inset.

$60 \mathrm{~Pa},-200 \mathrm{~V}$ bias

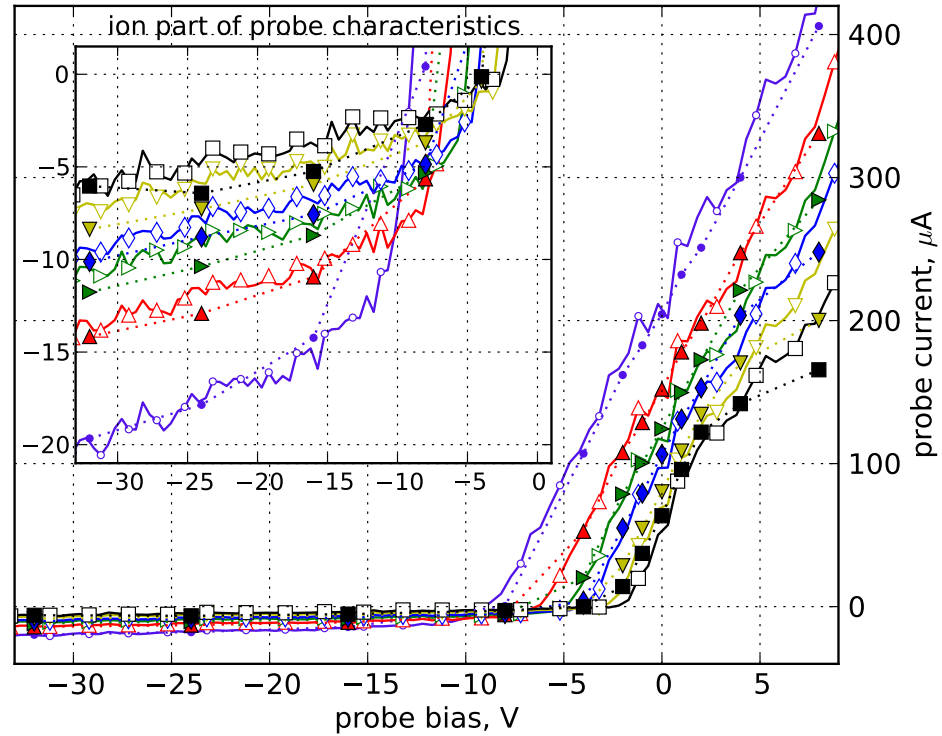

Figure 5.6: Comparison between simulated and measured probe characteristic for $60 \mathrm{~Pa}$ and $-200 \mathrm{~V}$ bias. The ion part of probe (scales: $\mu \mathrm{A}$ vs $\mathrm{V}$ ) characteristic is shown in the inset. The line legend is the same as in Fig. 5.5. 


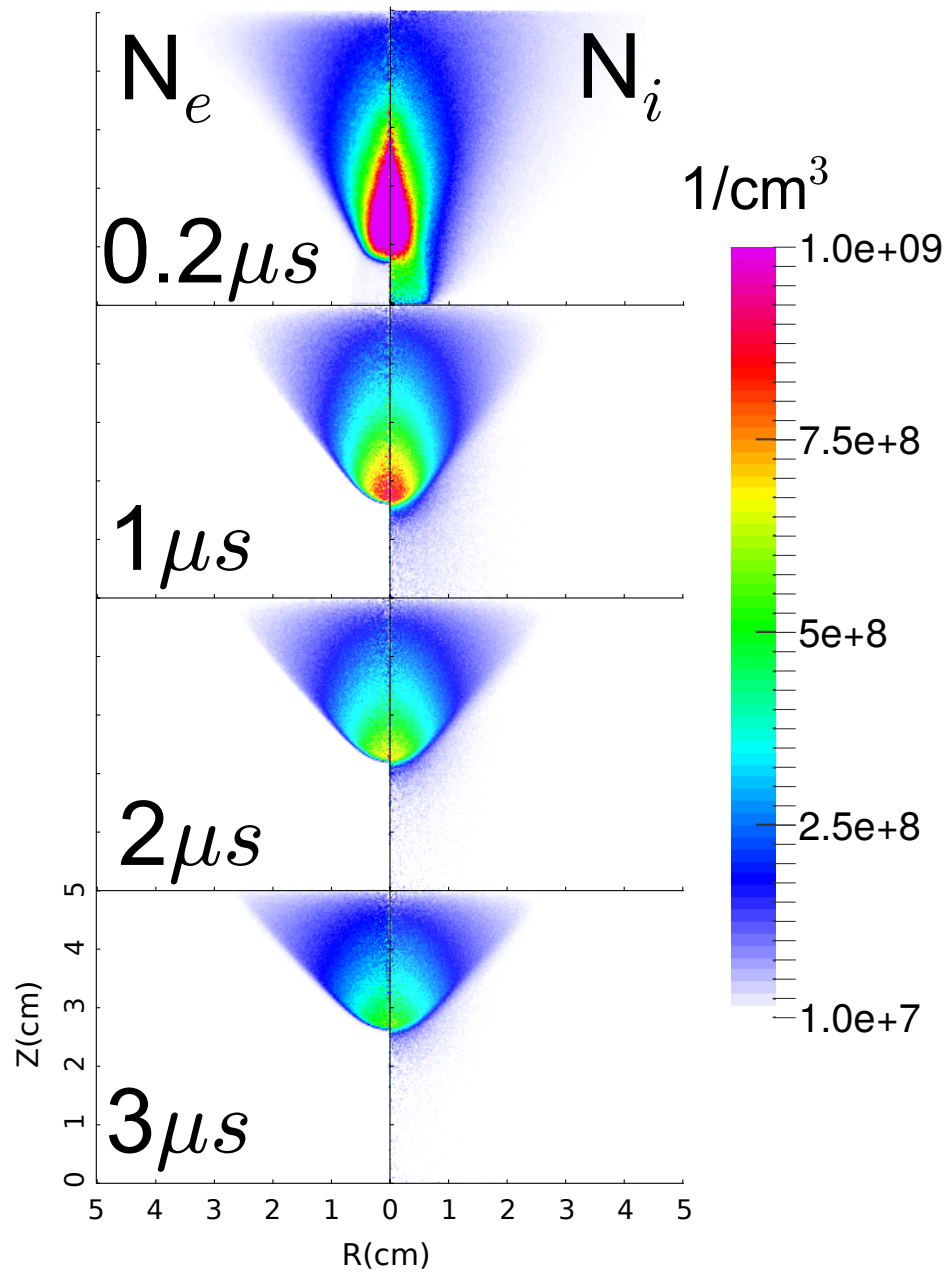

Figure 5.7: Electrons and ions (summed over ions types) densities for the different moments of time from UV pulse start. Simulation for $30 \mathrm{~Pa} \mathrm{H}_{2},-200 \mathrm{~V}$ bias between electrodes without probe in the chamber. 


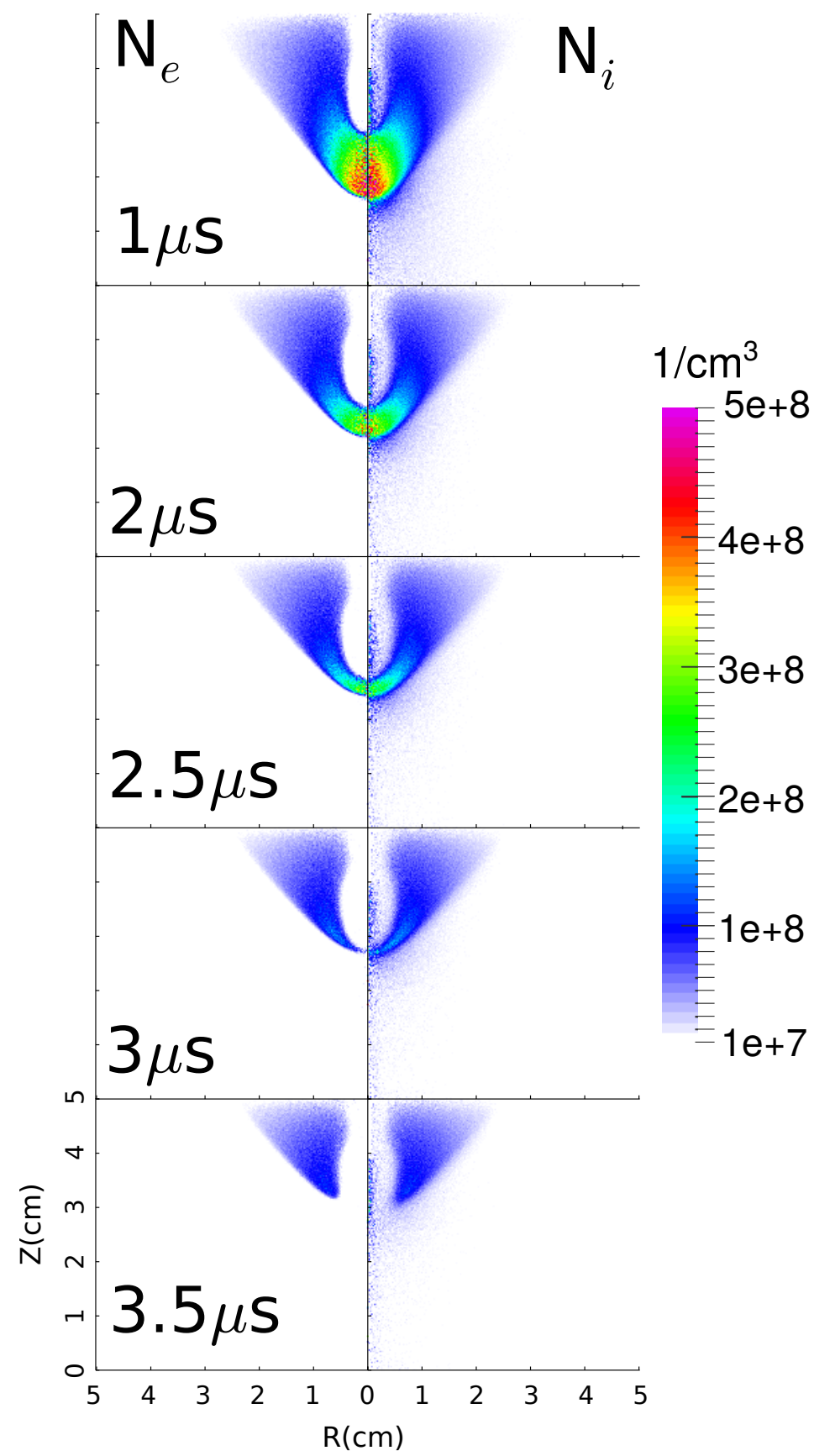

Figure 5.8: Electrons and ions (summed over ions types) densities for the different moments of time from UV pulse start. Simulation for $30 \mathrm{~Pa} \mathrm{H}_{2},-200 \mathrm{~V}$ bias between electrodes and $-32 \mathrm{~V}$ on the probe. 


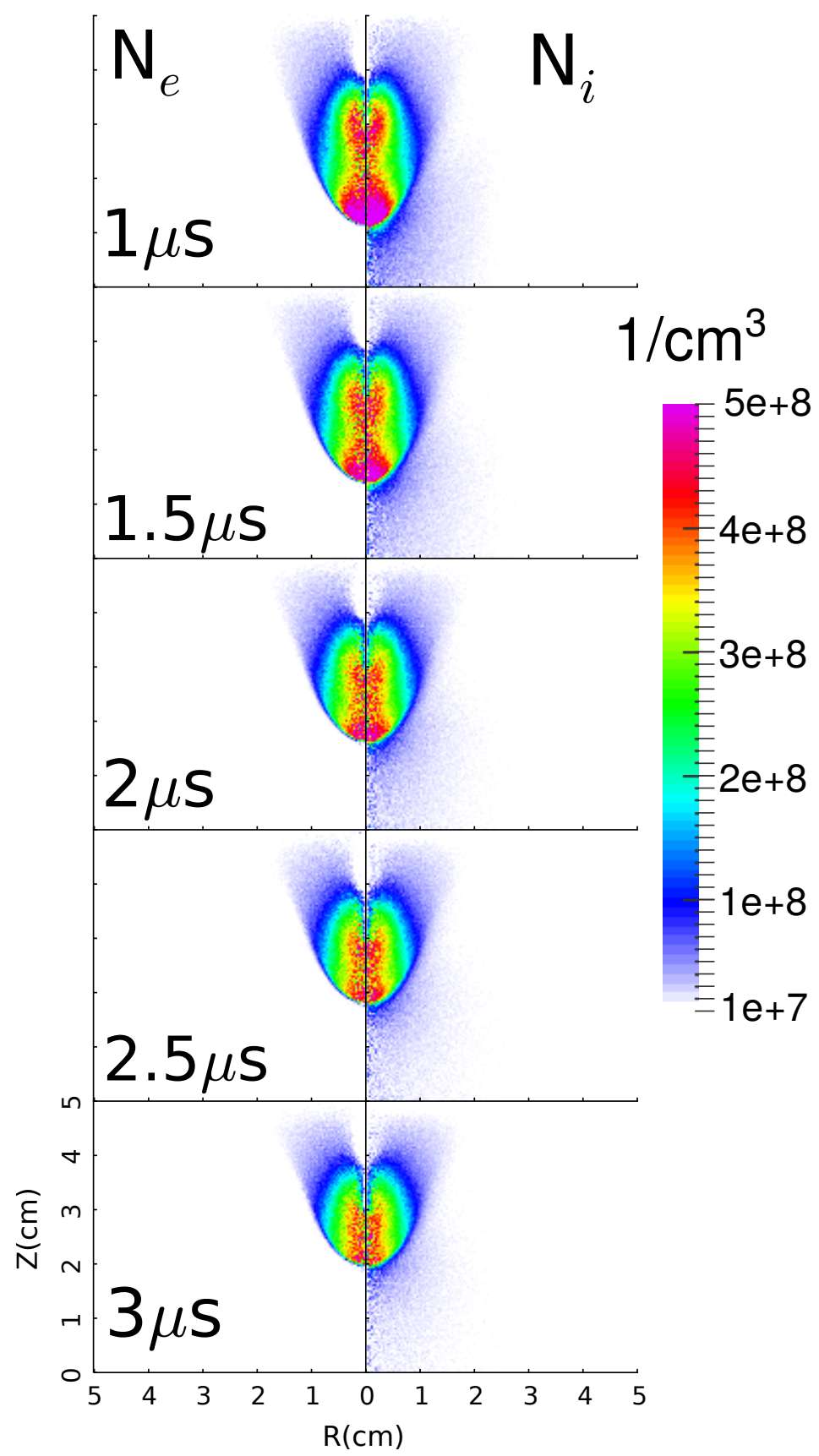

Figure 5.9: Electrons and ions (summed over ions types) densities for the different moments of time from the start of the UV pulse. Simulation for $60 \mathrm{~Pa} \mathrm{H}_{2},-200 \mathrm{~V}$ bias between electrodes and $-4 \mathrm{~V}$ on the probe. The probe current for this condition changes sign from positive at $1.5 \mu \mathrm{s}$ to negative at $3 \mu \mathrm{s}$, despite the plasma completely covering the probe. 


\title{
Chapter 6
}

\section{Exploring the electron density in plasma induced by EUV radiation: \\ II. Numerical studies in argon and hydrogen}

\begin{abstract}
We used numerical modeling to study the evolution of EUV-induced plasmas in argon and hydrogen. The results of simulations were compared to the electron densities measured by microwave cavity resonance spectroscopy. It was found that the measured electron densities can be used to derive the integral amount of plasma in the cavity. However, in some regimes, the impact of the setup geometry, EUV spectrum, and EUV induced secondary emission should be taken into account. The influence of these parameters on the generated plasma and the measured electron density is discussed.
\end{abstract}

\subsection{Introduction}

In the semiconductor industry, the photolithography process is used to create patterns on silicon wafers, an important step in microchip production. Due to diffraction, the characteristic pattern dimensions depend on the illuminating wavelength. However, it is becoming increasingly complicated to further reduce printed feature sizes with deep ultraviolet (DUV) light sources. As a result, extreme ultraviolet (EUV) lithography, operating at a wavelength of $13.5 \mathrm{~nm}$, is expected to be used to print integrated circuits with very high resolution features.

For various reasons, a buffer gas is frequently used in both laboratory and industrial EUV light sources. However, even at low pressure (1 .. $30 \mathrm{~Pa}$ ), EUV absorption leads to EUV induced plasma formation. There is a strong need for reliable diagnostics of EUV induced plasmas, because the interaction of an EUV induced plasma with the chamber interior and optical elements can lead to various plasma induced processes, such as surface etching, accelerated deposition of overlayers, and oxidation, depending on the exact constituents of the background gas [6,89].

It was shown in [90] that it is possible to measure the electron density of an EUV induced plasma, at relevant background gas pressures, by measuring the resonant frequency shift of a microwave cavity that contains the plasma. Although this is a highly sensitive method, it only allows the field average [91] electron density inside the cavity to be determined.

Nevertheless, it is interesting to know the spatial distribution of plasma in the cavity, the electron temperature and other plasma parameters. Unfortunately, measuring all the relevant parameters experimentally is difficult due to the transient nature of the EUV induced plasma. For example, the insertion of a probe can yield unreliable estimates of plasma parameters for EUV induced plasmas, since, during and after the EUV pulse, the probe signal is heavily distorted [92, 93]. 
In this paper, we use a particle-in-cell model of the EUV-induced plasma to determine the spatio-temporal distribution of the electron density, the electron-ion balance, and the influence that some confounding factors may have on the spatio-temporal distribution of the plasma. Specifically, we studied how the changes in buffer gas, EUV intensity, EUV spectrum, and secondary electron emission change the plasma in the cavity.

We found that the EUV-induced plasma can be in a distinct "charged" regime. In this regime (as opposed to the quasi neutral regime, that corresponds to usual plasmas, such as glow discharges) the quasi neutrality is violated everywhere in the plasma. This regime forms due to the escape of fast photoelectrons from the plasma to the cavity walls. That leaves a highly charge imbalanced plasma. Such a plasma is not well suited for study with the microwave cavity method. However, it happens that such a regime is on the lower boundary of the methods sensitivity. Therefore, it can be easily detected and avoided.

We evaluated the accuracy of the simulations by comparing of the simulated field averaged electron density with the experimentally measured electron densities. We show that in the quasineutral plasma regime, the microwave cavity resonance shift provides an accurate tool for estimating plasma parameters, using only the spatial profile of the cavity mode and the EUV beam profile as input factors. In the charged regime, similar accuracy can only be obtained if on has knowledge of the EUV spectrum and secondary electron emission among other factors.

\subsection{Experiment}

The experimental setup is described in detail in Ref. [90]. Therefore, here, we provide a minimal outline of the experimental procedure.

The plasma is ignited in a resonant cavity (see Fig. 6.1) by a beam of EUV radiation, which is introduced along the axis of the cylindrically symmetric cavity with an aperture and beam guide to prevent residual EUV from being incident on the cavity walls. The EUV radiation is produced by a xenon based discharge source and propagated through a spectral purity filter (SPF) to limit the spectrum to photon energies to the range of 70 to $120 \mathrm{eV}$. The spectrum of the source after transmission through the SPF is shown in Fig. 6.2.

The EUV power is monitored using a sensitive thermocouple, attached to a copper disk, located approximately $2 \mathrm{~cm}$ behind the cavity.

As described earlier, the average electron density in the resonant cavity was determined by measuring the resonant frequency of the microwave cavity (see [90] for details).

\subsection{Model}

The dynamics of the EUV-induced plasma was simulated using a two dimensional ( $r z$ cylindrically symmetric) particle-in-cell plasma model with Monte Carlo collisions (PIC-MC). Both electrons and ions are represented by particles to describe the dynamics of the plasma accurately. The model follows the general PIC scheme, described elsewhere [28]. 


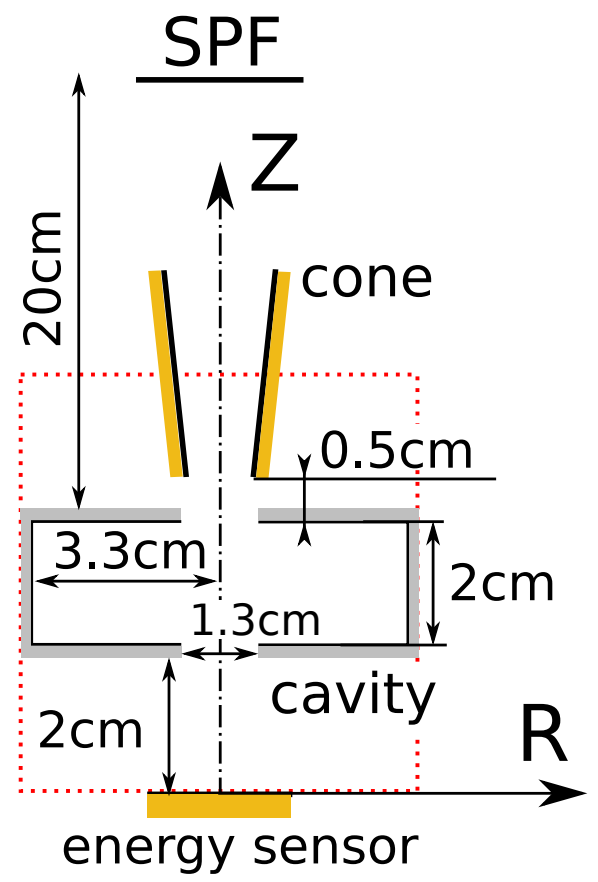

Figure 6.1: Configuration of the experimental chamber. The EUV radiation is introduced via a spectral purity filter (SPF) along the symmetry axis of the cavity.

\subsubsection{Photoionization}

The absorption of EUV radiation in the volume is included as a source of slow ions and fast electrons. To simulate photoionization, we use the measured spatial (axially averaged) and temporal profiles of the EUV pulse (see Fig. 6.5 and Fig. 6.9). We assume that the spatial profile and spectrum of the EUV source does not vary during EUV pulse.

The spatial and temporal coordinates of electron-ion pairs that are created by ionization events are added to the simulation domain using a probability distribution function that is weighted by the spatial and temporal profiles of the EUV pulse. The energy of the injected electrons is set to the difference between the photon energy, which is selected according to the EUV spectrum, and the ionization potential of the gas species $\left(\mathrm{H}_{2}\right.$ or $\left.\mathrm{Ar}\right)$. The the power spectral density of the EUV beam is recalculated along the $z$ axis to take into account absorption by the gas between SPF and cavity entrance using the Lambert-Beer law.

The electrons are emitted preferentially in the $r$ plane, with a distribution function

$$
P(\theta) \sim 1-\beta \frac{1}{4}\left(3 \cos ^{2} \theta-1\right)
$$

where $\theta$ is the angle between electron velocity and EUV beam direction and $\beta$ is the anisotropy factor. This form of angular distribution corresponds to photoionization by unpolarized light [94]. For hydrogen we use $\beta=2$, as this agrees with experiments in the energy range of the mea- 
surements. For argon, we use data from [95, 96] for photon energies below $95 \mathrm{eV}$, and $\beta=1.3$ above $95 \mathrm{eV}$.

The energy dependent photoionization cross-sections are taken from [36] and [97] for $\mathrm{H}_{2}$. For argon there are several measurements (e.g. [98], [99] and [100]). We followed the recommendation of [101] and used data from [98] for the total absorption cross-section. The cross-section for photoionization to $\mathrm{Ar}^{++}$is taken from [102].

\subsubsection{EUV the spectrum}

To simulate the EUV plasma ignition, the spectrum of the EUV radiation must be taken into account. Unfortunately there is no simple experimental method to control the radiation spectrum in the relevant broad range of VUV to EUV photon energies $(15.4-120 \mathrm{eV})$.

Therefore, as input for the simulations, we have used the source spectrum, measured before transmission through the SPF, convolved with the measured transmission spectrum of the Si: $\mathrm{Zr}$ SPF. This results in the EUV spectrum shown in Fig. 6.2.

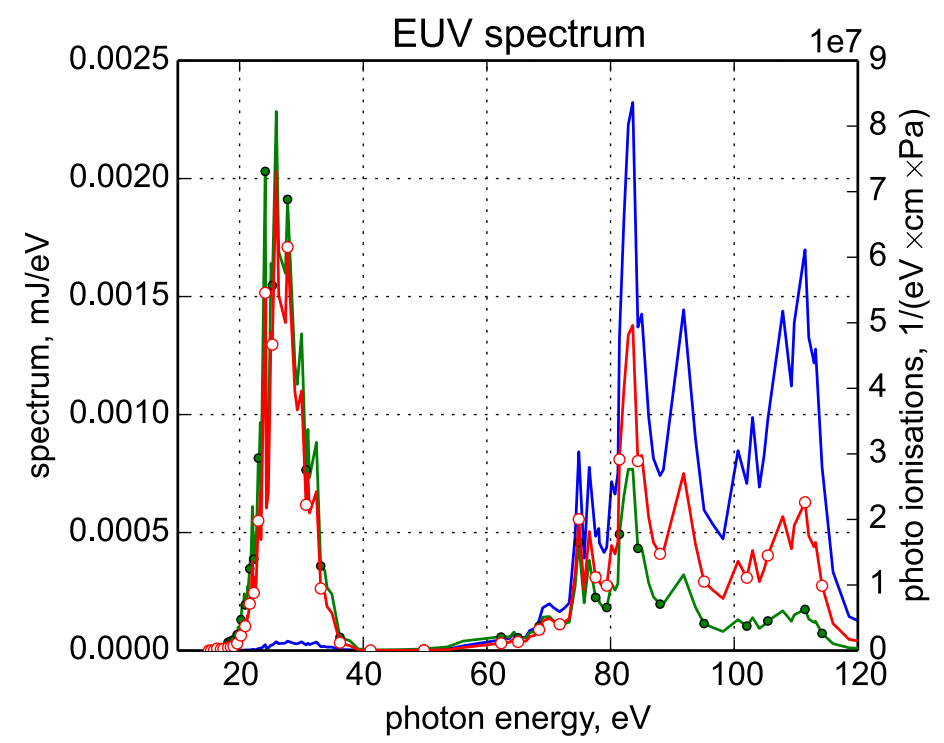

$\because \mathrm{H}_{2}$ ionisation $\times 10 \quad \propto$ Ar ionisation

- spectrum after SPF

Figure 6.2: EUV power spectral density $(I(E))$ spectrum as used in the simulations. The right axis shows the number of direct photoionization events due to transmission of $0.044 \mathrm{~mJ}$ EUV through $1 \mathrm{~Pa}$ of gas, i.e. $I(E) \cdot N[1 \mathrm{~Pa}] \cdot \sigma(E) / E$, where, $\sigma(E)$ is the photoabsorption cross-section, $E$, the photon energy, and $N[1 P a]$ the number density .

Fig. 6.2 shows that the transmission of the SPF filter in the $20-40 \mathrm{eV}$ range, which is in the range of $1 \%$ of the total pulse energy, results in significant additional photoionization. This additional photoionisation is comparable to the direct photoionization due to the high energy 
part of the spectrum (i.e. $60-120 \mathrm{eV}$ ), because the photo absorption cross-section is very large in the $20-40 \mathrm{eV}$ range compared to the $60-120 \mathrm{eV}$ range.

Although the presence of the radiation in the $20-40 \mathrm{eV}$ range has been confirmed by measurements, the absolute accuracy of the transmitted spectrum is not accurately known. This introduces an additional uncertainty into the simulations. Because the accuracy of the measurements of the EUV dose per pulse is also not know, we choose to keep spectrum shape constant and vary the EUV dose. This approach has the advantage that the integral accuracy of the simulations can be estimated by the difference between the measured EUV energy per pulse and that required for good agreement between experiments and simulations for both gases (i.e., we require that the same EUV power results in agreement between model and experiment over a range of experimental conditions).

\subsubsection{Secondary electron emission and influence of chamber configuration}

Emission of slow electrons from the walls to the plasma due to any reason can significantly decrease the plasma potential, or even lead to the collapse of the plasma sheath [103] leading to effective energy transfer from the electrons to the walls. Therefore, it is important to take into account secondary electron emission (SEE) from the the surfaces that are exposed to the plasma.

For the considered setup, energy is only injected into the plasma during the EUV pulse via photoionization and photoelectrons emission from irradiated surfaces. Therefore, the decrease of the plasma potential or sheath collapsed due to SEE can result in significant loss of fast electrons to the walls. Hence, the maximum number of ionization events also decreases.

\section{Secondary electron emission}

In order to estimate the role of emission from the aluminum cavity walls due to electron impact, we have considered three cases. The first corresponds to no secondary electron or ion induced emission from the walls. In the second case, electron emission due to both electron and ion impact is taken into account. The data for aluminum shows a significant spread of possible emission yields. Nevertheless, for the energy range of primary photoelectrons (e.g. $50-75 \mathrm{eV}$ ) the reported values are typically larger than $0.3[104,105]$ so we use the yield reported for clean aluminum in Ref. [105].

The yield also significantly depends on the surface conditions. For aluminum with a native oxide, the emission yield [106] is significantly higher than for pure aluminum and corresponds to the yields found for dielectric materials $[107,108]$. Therefore, in the third case, the yield for aluminum with a native oxide is used. The yield is estimated from Ref. [106] for energies below $25 \mathrm{eV}$, and extrapolated linearly for energies above $25 \mathrm{eV}$.

We do not discriminate between backscattered electrons and true secondary electrons, because, for our study, only the total flux from the surface is important. In the model, we assume that most of the emitted electrons are cold, i.e. they have a significantly lower energy compared to the energy of the impacting electrons. However, for electron with energies below $10 \mathrm{eV}$ this approximation is not valid, due to the higher probability of elastic backscattering. But, these backscattered electrons do not have sufficient energy to ionize more of the background gas, if not accelerated by a plasma potential. Therefore, the additional error due to this approximation 
is expected to be small.

\section{influence of chamber configuration}

The configuration of the experimental setup is presented in Fig. 6.1. The SPF filter, copper cone and copper disc of energy sensors emit secondary electrons under EUV irradiation. But, these electrons do not contribute directly to the ionization inside cavity, because the number of high energy secondaries is small and the electron flux is not focused.

To estimate the influence of this effect, we have simulated two geometrical configurations: a large volume, which includes the cone and disc (the dotted rectangle in Fig. 6.1), and a small volume, which includes only the cavity with periodic boundary conditions at the cavity openings. We have not included the full length of the cone and SPF into simulations, because the aspect ratio of the cone diameter to length is very small (i.e. 0.05).

\section{Photo electron emission from surface}

In order to model the configuration that includes the cone and copper disc, photoelectron emission should be taken into account. The energy spectrum of photoelectrons emitted from the copper disk and cone surfaces are calculated from [39]:

$$
P(E) \sim \frac{E}{(E+W)^{4}}
$$

Here $P(E)$ is the probability of emitting an electron with energy, $E$, from a surface with a work function, $W$. For copper $W=5 \mathrm{eV}$ [40]. For electrons emitted from the surface, we assume an angular dependence given by a cosine emission law [39]. The effective photoelectron yield from copper was set to 0.05 electron/photon [109].

\subsubsection{Grid resolution}

The grid resolution was chosen to resolve the Debye length. To estimate the minimum Debye radius in simulations, we assumed a temperature of $0.5 \mathrm{eV}$ and an estimated maximum plasma density. The maximum plasma density was estimated from direct photoionization (i.e., Fig. 6.2), increased by the maximum possible number of impact ionizations that the primary electrons could generate, i.e., for a $90 \mathrm{eV}$ photon, the photoelectron has an energy of $74.6 \mathrm{eV}$, therefore, the maximum number of ions produced by that electron is 5 , increasing the plasma density by a factor of 5. Note, however, that, in the simulation, the contribution of fast photoelectrons is significantly smaller due to the contribution of inelastic collisions, and that many photoelectrons escape to the wall before generating the maximum number of ions.

\subsubsection{Cross-sections sets}

Two independent cross-section sets are used for modelling hydrogen and argon. Both sets consists of electron and ion collisions with corresponding neutrals. The collisions between plasma species and three body processes are neglected due to their low probability under the conditions considered here. 
We make use of the procedure described in [42] to perform Monte Carlo collisions with the background gas. We tested the consistency of our implementation by modeling swarm experiments and found good agreement with experimental values [54] for the first Townsend electron ionization coefficient, the electron mobility, for $\mathrm{H}^{+}$and $\mathrm{H}_{3}^{+}$mobility in hydrogen [55], and for $\mathrm{Ar}^{+}$and $\mathrm{Ar}^{++}$mobility in argon $[110,111]$.

\section{Hydrogen}

To accurately model electron collision related processes in hydrogen discharges with a Monte Carlo (MC) model, one needs to take into account the differential cross-sections for ionization and excitation processes. As described in detail in [64], the particular choice of the angular dependence of cross-sections significantly influences the simulation results.

For electron $-\mathrm{H}_{2}$ collisions we adopt a set of cross sections found in Ref. [64] with small corrections. We use an experimentally determined doubly differential cross-section for the electron impact ionization of hydrogen $[57,58]$. Electron elastic scattering and hydrogen electronic excitations, and angular scattering data taken from [46].

The set of cross-sections for collisions between ions and hydrogen is based on [62] because this set provides good agreement with swarm data for ions in hydrogen.

We neglect the formation of $\mathrm{H}^{-}$, because the cross-section of dissociative electron attachment is very low, and the density of vibrationally excited hydrogen molecules too low to significantly contribute to the production of $\mathrm{H}^{-}$.

\section{Argon}

For electron - Ar collisions we adopt a set of cross-sections found in Ref. [112, 113]. We add electron impact ionization of Ar to $\mathrm{Ar}^{++}$from Ref. [114] to the cross-sections set. We use differential cross-section data for electron impact ionization from Ref. [115]. The data from Ref. [115] covers incident electron energy range $17-30 \mathrm{eV}$. To the authors knowledge there is no systematic differential ionization cross-section measurements for incident electrons in the energy range of $30-100 \mathrm{eV}$. Hence, for energy range above $30 \mathrm{eV}$, we use empirical formulas from Ref. [116].

We neglect ionization via the metastable argon exited state. The density of metastables produced in one EUV pulse is comparable to the plasma density (e.g. $\sim 10^{9}-10^{10} 1 / \mathrm{cm}^{3}$ ). The maximum of cross-section for electron impact ionization of the metastable argon exited state is approximately $10^{-15} 1 / \mathrm{cm}^{2}$ [117]. Therefore, the maximum corresponding ionization frequency can estimated as $\nu_{i}=n \sigma v \sim 2 \cdot 10^{3} 1 / \mathrm{s}$. The simulated plasma evolution is less than $10 \mu \mathrm{s}$, yielding an upper bound of 0.02 ionization events per electron for the step wise ionization. This is negligible compared to the direct EUV ionization and ionization by fast photoelectrons. Moreover, there is no pulse-to-pulse accumulation because the time between EUV pulses is larger than metastable's lifetime due to quenching on the chamber walls.

The set of cross-sections for collisions between $\mathrm{Ar}^{+}$and argon is based on [118]. For collisions between $\mathrm{Ar}^{++}$and argon we use data from [119]. 


\subsubsection{Calculation of the field average electron density}

In order to compare the model results with experimental measurements, the simulated spatiallyresolved electron density was averaged over the spatial profile of the resonant microwave cavity mode as follows.

$$
\left\langle n_{e}\right\rangle=\frac{\int n_{e} \vec{E}^{2} d V}{\int \vec{E}^{2} d V}
$$

The magnitude of the microwave field was very small ( $\mathrm{mV}$ range), therefore, it was not included in PIC-MC simulations.

\subsection{Results and discussion}

The ignition and decay of the EUV induced plasma was simulated for a series of argon and hydrogen pressures (i.e. $1 \mathrm{~Pa}, 5 \mathrm{~Pa}, 10 \mathrm{~Pa}$ ) to compare with experimental results. The best agreement with experiments for both gases and all considered pressures was obtained in simulations where we have included the larger chamber configuration, and used a spectrum that includes a VUV contribution. The comparison between simulated field averaged electron density and experimental measurements is presented in Fig. 6.3 and Fig. 6.4.

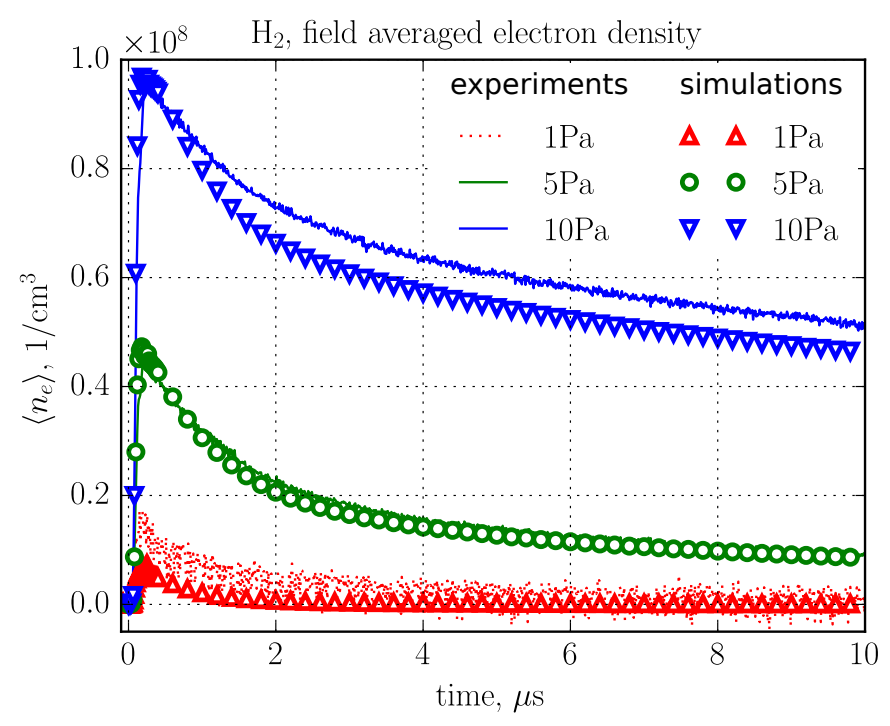

Figure 6.3: Comparison between the simulated (symbols) and measured (lines, data from part I) time dependence of field averaged electron densityfor 1 (red), 5 (green), and 10 (blue) Pa hydrogen.

In these simulations, we have decreased the EUV energy per pulse to $0.035 \mathrm{~mJ} /$ pulse from the experimentally measured value of $0.044 \mathrm{~mJ} /$ pulse. This difference, in combination with $\sim 20 \%$ relative errors for the field averaged electron densities (i.e. in Fig. 6.3 and Fig. 6.4), yields a 50\% 


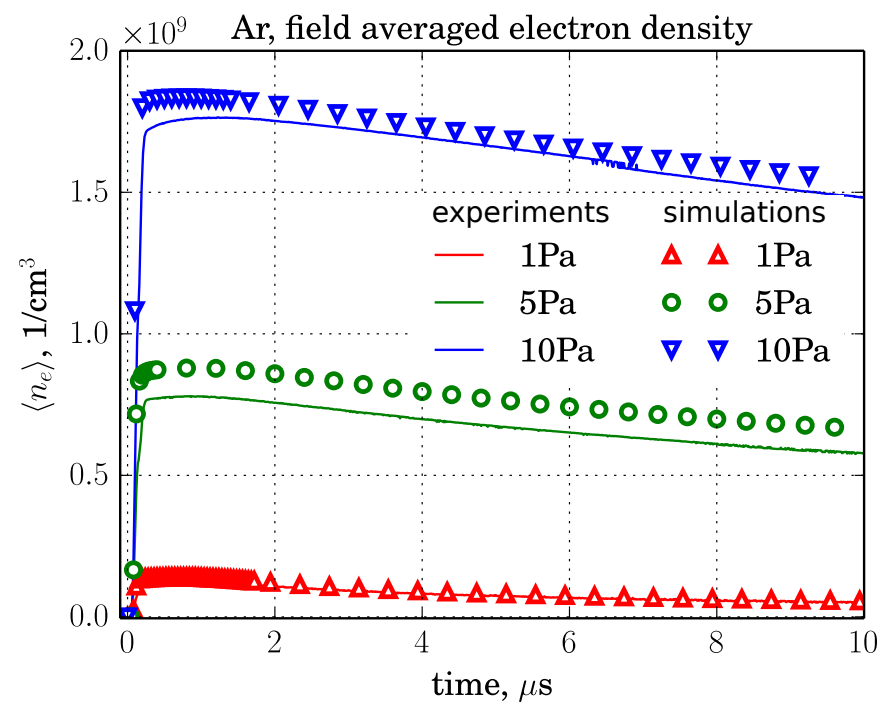

Figure 6.4: Comparison between the simulated (symbols) and measured (lines, data from [120]) time dependence of field averaged electron density for 1 (red), 5 (green), and 10 (blue) Pa argon.

cumulative uncertainty, which is comparable to the $\sim 30 \%$ error margin of of the experiment [90].

\subsubsection{Relation of field averaged electron density and simulated values}

The cavity method allows the field averaged electron density to be determined. But, the relationship of this quantity to the actual plasma density is not straightforward. During the first $1 \mu \mathrm{s}$, the simulated plasma is much denser than that measured by the cavity. However, as the plasma expands, the difference between simulated plasma density and cavity measurements becomes smaller. For $5 \mathrm{~Pa} \mathrm{H}_{2}$ at $10 \mu \mathrm{s}$, the ratio between the simulated plasma density and that measured by the cavity is only about a factor of two (see Fig. 6.5).

Nevertheless, the plasma density measured by the cavity is very useful to estimate the amount of the plasma in the cavity. Let us define the effective volume of the cavity as ratio of the full number of electrons in the cavity to the cavity measured electron density i.e. $V_{\text {eff }}=$ $\int n_{e} d V /\left\langle n_{e}\right\rangle$. In simulations, this quantity varies only by about a factor of two during the $10 \mu \mathrm{s}$ simulation window (see insert in Fig. 6.5). Moreover, the estimation of the effective volume, based on the EUV spatial profile (as an approximation to the EUV plasma distribution), and the spatial profile of the microwave cavity mode (see Part I) agree well with the simulated values (dashed black line in the insert in Fig. 6.5)

Therefore, the amount of EUV induced plasma can be estimated from the measured cavity response with a relative accuracy of about $30 \%$. Hence, the amount of absorbed radiation can be estimated with similar accuracy, if the other factors (see below) that effect plasma formation are eliminated. 


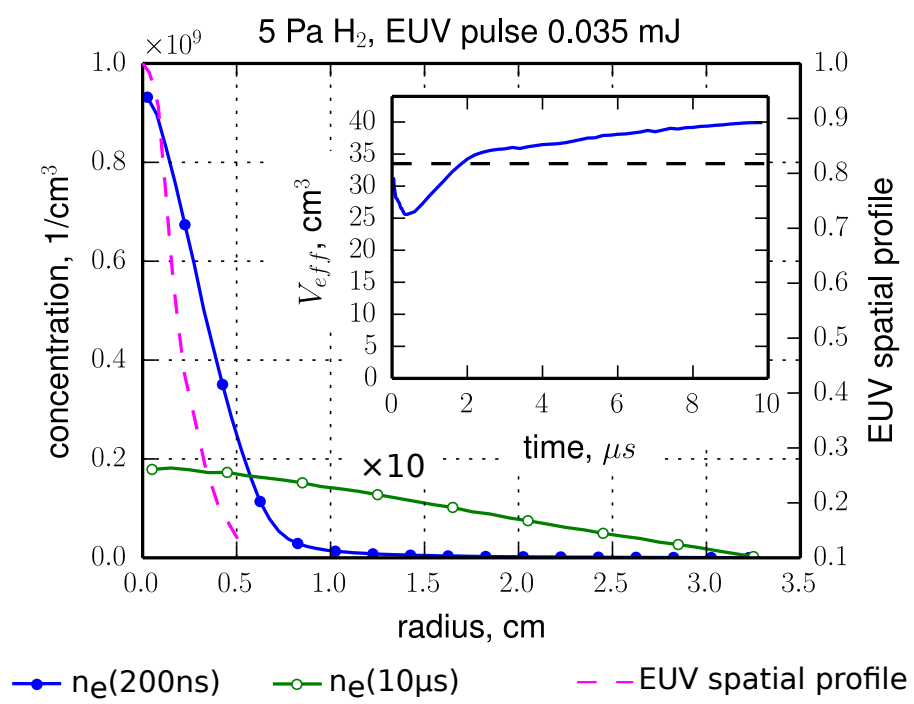

Figure 6.5: Radial distributions of electron density in the cavity for $5 \mathrm{~Pa} \mathrm{H}_{2}$ and the EUV spatial profile that was used for all simulations. The insert shows the time dependence of the effective volume probed by the microwave mode. The simulated volume is a solid blue line, while the dashed black line corresponds to analytical estimation (see text).

The simulated evolution of the effective volume is non monotonic in time. A similar effect was observed in experiments via the ratio of the electron densities measured using 010 and 110 cavity modes [121]. However, in simulations, the decrease of the effective volume just after the EUV pulse is caused by the convolution of the plasma density with the spatial profile of the microwave mode in the cavity.

\subsubsection{Effect of VUV part of the spectrum}

As discussed in section 6.3.2, the EUV spectrum after passing through the SPF (see Fig. 6.2) has two distinct regions: a low energy $(20-40 \mathrm{eV})$ VUV part, and a high energy $(60-120 \mathrm{eV})$ EUV part. Our simulations show that the VUV contribution is needed to produce consistent results for both gases with the same amount of the radiation dose per pulse. This is because the average number of ionization events per absorbed photon can be larger than one if the emitted fast photoelectron produces addition ionization events.

In our simulations, the average number of ionizations per absorbed EUV photon was observed to be approximately one for $\mathrm{H}_{2}$ and two for Ar. To obtain the observed electron densities without including ionization due to VUV, the EUV pulse energy must be increased and set to different values for argon and hydrogen.

\subsubsection{Effect of secondary electron emission due to EUV radiation}

Our simulations of the larger, more complete geometry shows that electron emission from the cone walls and copper disk change of the plasma potential. The large EUV induced secondary 


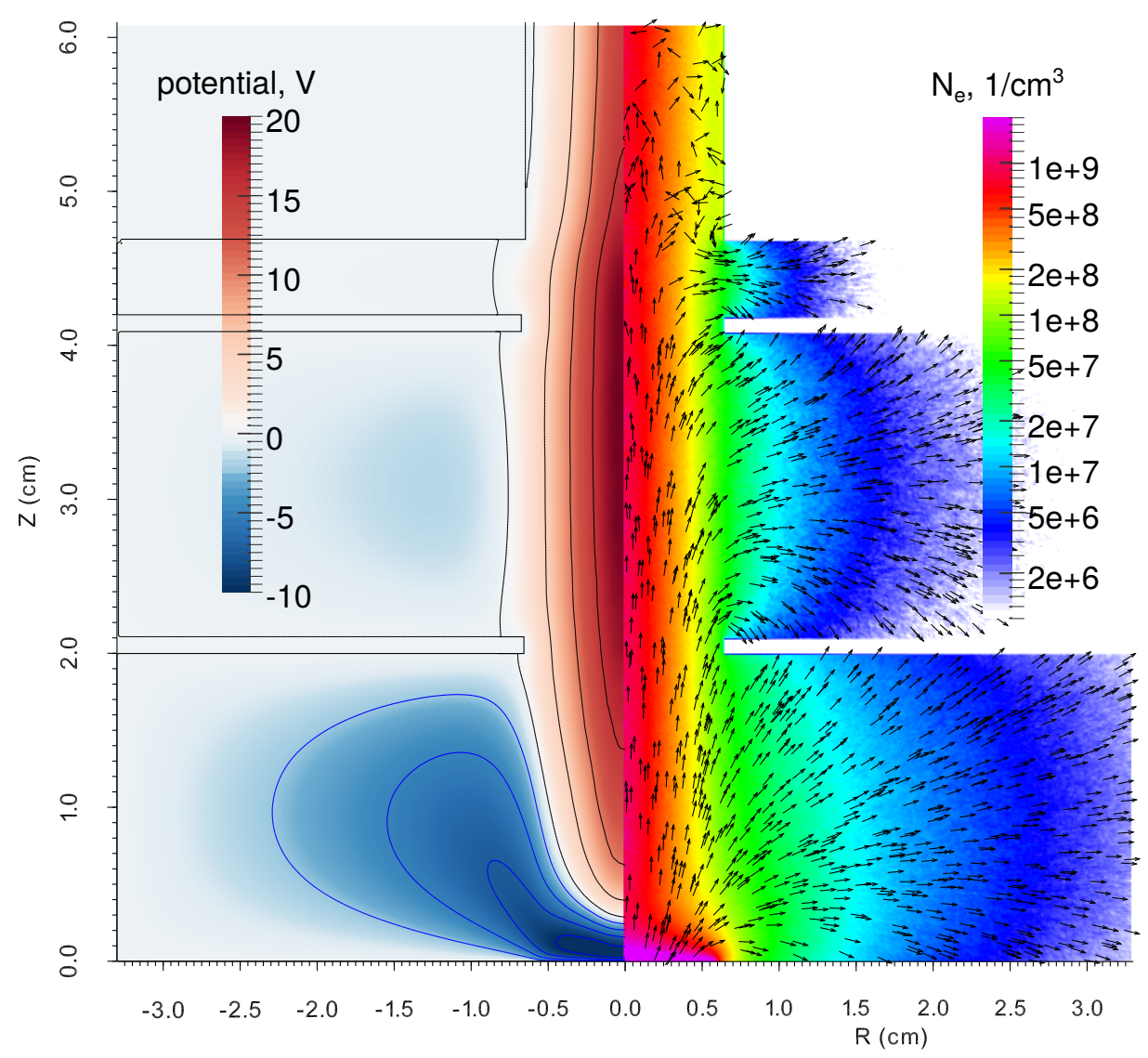

Figure 6.6: Distribution of the potential, electron density and current direction in the simulation domain 100 ns (see Fig. 6.9), $5 \mathrm{~Pa} \mathrm{H}_{2}$. The arrows shows the direction of the electron current. The electrons are drained from the copper disc $(Z \sim 0)$ into the cavity, leading to a decrease of the plasma potential (see Fig. 6.7).

electron emission from the copper disk leads to the formation of the space charge potential well during EUV pulse, see Fig. 6.6. The electron current during the pulse on the symmetry axis of the system is directed from the copper disk towards the cavity, thus replacing a large portion of the fast electrons that reach cavity walls. A comparison between the potential in the cavity for $5 \mathrm{~Pa} \mathrm{H}_{2}$ for both geometrical configurations is presented in Fig. 6.7. For these simulations, the electron induced electron emission from cavity walls was set to zero.

Interestingly, the average electron density decreases for argon gas, but increases for hydrogen, compared to simulations of the cavity only. In the case of hydrogen, the plasma potential is smaller than for argon, due to a lower plasma density. That, in turn, leads to a significant loss of electrons to the cavity walls. Electrons emitted from the cone and copper disc are accelerated into the plasma, compensating for a fraction of the escaped electrons and increase the electron density.

In contrast to hydrogen, the argon plasma is significantly denser, due to the order of magni- 


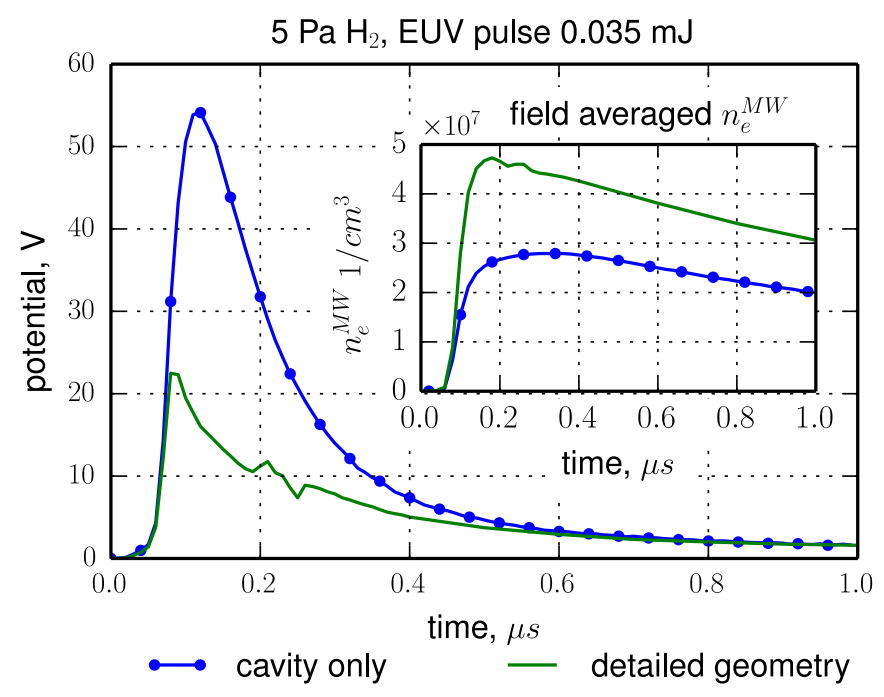

Figure 6.7: Comparison of the potential in the center of the cavity as function of time for $5 \mathrm{~Pa}$ $\mathrm{H}_{2}$. The large geometrical configuration includes secondary electron emission from the energy sensor, which leads to a decrease of the potential in the cavity. A comparison between the field averaged electron densities in the cavity is shown in the insert. A non-monotonic decrease of the plasma potential for the large configuration is related to SE emission from energy sensor and charge redistribution in the cavity. During the EUV pulse, SE emission leads to a significant decrease of the plasma potential, since the emitted SE electrons replace the escaped primary photoelectrons. Just after the EUV pulse there are no SEs emitted, but some fast photoelectrons are still present in the plasma, therefore, once these fast electrons escape to the walls the plasma potential increases temporally.

tude larger absorption of EUV for the same pressure (see Fig. 6.2). Hence, for argon, the relative charge imbalance between electrons and ions is much smaller than for hydrogen. Therefore, these electrons change the potential landscape of the plasma, increasing the probability of fast photoelectrons escape to the walls and reducing the average number of ionizations per fast electron.

\subsubsection{Influence of electron induced secondary emission from cavity walls}

As discussed in section 6.3.3 one can expect a significant secondary electron emission yield from the cavity walls under electron impact. However, the inclusion of this effect in the simulations has a small impact on the plasma formation in the cavity (below $5 \%$ for the field averaged electron density).

It is explained by changes in the plasma potential (see Fig. 6.8). Most ionization occurs in the region corresponding to the EUV beam. Electrons that escape from the plasma to the region between the beam and the cavity walls encounter the slow electrons produced on the cavity walls due to electron impact emission. That lead to formation of a local minimum in the plasma potential. 


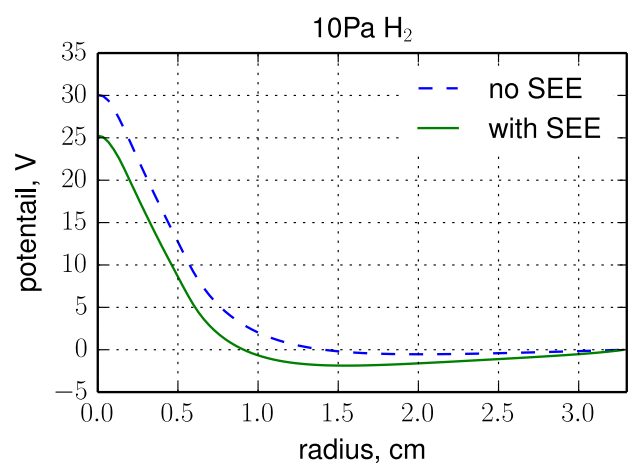

Figure 6.8: Comparison of the radial dependence of the plasma potential at the center of the cavity with (green) and without SEE (blue) for $10 \mathrm{~Pa} \mathrm{H}_{2}$ at $80 \mathrm{~ns}$ after pulse start.

Hence, the potential barrier that electrons in the beam need to overcome in order to escape is similar for both simulations that include and neglect electron induced emission from the cavity wall. Moreover, the potential near cavity wall during the pulse prevents the emitted secondary electron from entering the plasma, thus significantly limiting the influence of SEE on the plasma formation.

\subsubsection{High charge imbalance plasma for $1 \mathrm{~Pa}_{2}$}

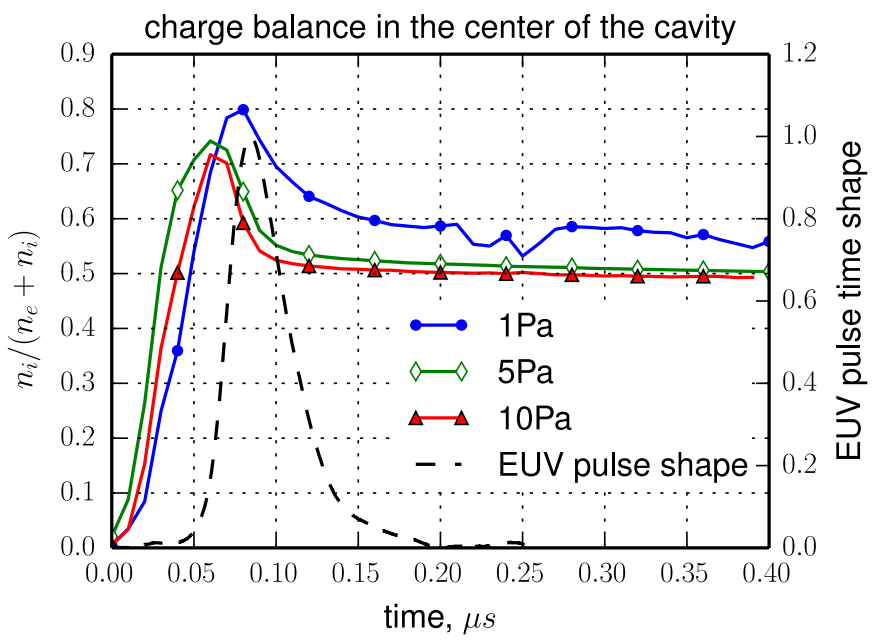

Figure 6.9: Evolution of the charge balance in the center of the cavity for the case of $\mathrm{H}_{2}$. The charge imbalance for the case of $1 \mathrm{~Pa} \mathrm{H}_{2}$ lasts significantly longer compared to other cases.

Interestingly, results for $1 \mathrm{~Pa} \mathrm{H}_{2}$, even with inclusion of the VUV part of spectrum, show 


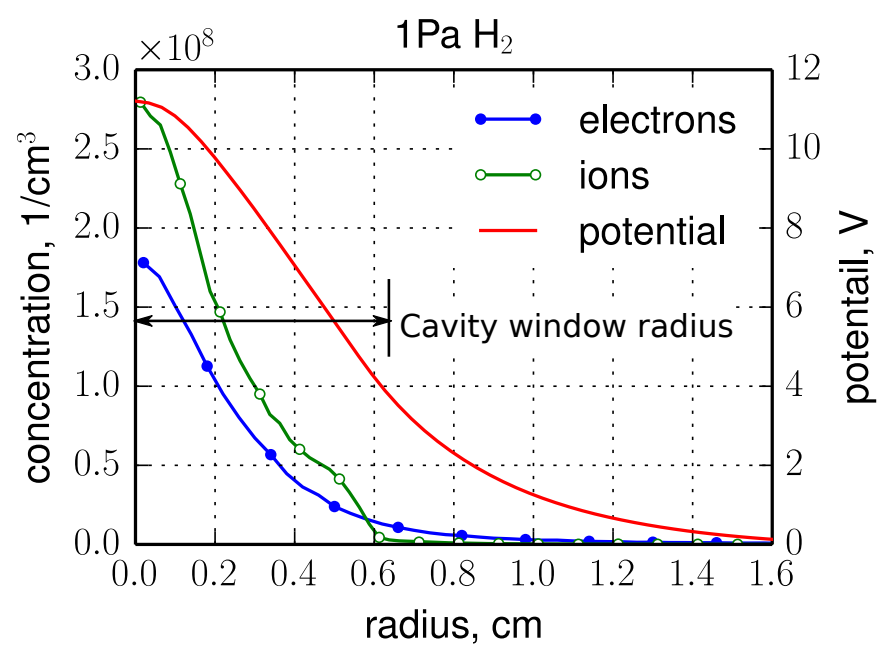

Figure 6.10: Radial distribution of electrons and ions in the center of the cavity at $150 \mathrm{~ns}$ after EUV pulse start.

a significant charge imbalance between ions and electron. Due to the low $\mathrm{H}_{2}$ pressure, the absorption of EUV radiation is low. The generated plasma has a peak density in the range of $2 \cdot 10^{8}-3 \cdot 10^{8} 1 / \mathrm{cm}^{3}$, see Fig. 6.10. This plasma can generate only a low plasma potential, that is not enough to confine high energy primary photoelectrons. As result, after the EUV pulse, a significant fraction of electrons leaves the plasma.

The ratio of the ion density to the sum of the ion and electrons densities as a function of time are presented in Fig. 6.9. For all simulated cases, in the beginning of the pulse, many fast electrons leave the plasma, creating an excess of ions. After the pulse, the simulation show that the plasma returns to quasi-neutrality (i.e. $n_{i} /\left(n_{i}+n_{e}\right) \sim 0.5$ ), except for the case of $1 \mathrm{~Pa} \mathrm{H}_{2}$. In the case of $1 \mathrm{~Pa}$, the generated plasma remains significantly charged, which leads to a faster decay, due to Coulomb repulsion on the time scale of $1 \mu \mathrm{s}$.

\subsection{Conclusion}

We simulated the evolution of an EUV induced plasma in a microwave resonant cavity. During simulations we observed that the cavity measured electron density can be used to estimate the integral amount of ionization in the cavity.

We show that these estimates are reasonably accurate ( $<30 \%$ for the considered conditions). This is even the case for estimates that use only the spatial mode profile of the microwave cavity mode and the EUV spatial beam profile. Therefore, a suitable microwave cavity can be used as a valuable diagnostics tool for EUV induced plasmas.

However, the cumulative uncertainties of the SPF transmission and the possible error margin in the measurements of the EUV source spectrum are important for the analysis of EUV plasma formation in hydrogen and argon. This is due to the rapid increase of the absorption cross- 
section with decreasing photon energy. Even $1 \%$ transmission of the SPF filter in the range of $20-30 \mathrm{eV}$ has a significant effect on the EUV plasma formation. Moreover, the same spectral range is very important for surface chemistry processes, because the photo-absorption crosssections are typically very large (e.g. the convolution of the cross-section with the spectrum can be similar for in-band EUV and out-of-band VUV).

The effect of VUV is likely to be significant, because most laboratory sources are discharge based with grazing incidence collector optics, which, without special care will have a significant amount of VUV. Therefore, in laboratory experiments, the EUV spectrum should be measured after the SPF filter, otherwise the remaining VUV radiation can induce a substantial systematic error.

Last but not least, it is important to take into account the EUV (or VUV) induced secondary electron emission (SE) form cavity and/or surroundings. This emission changes the potential distribution in the cavity and, hence, changes the generated plasma density, which further complicates the analysis of the cavity response.

Nevertheless, in case the radiation spectrum and secondary electron emission are known through additional measurements, the microwave cavity response may prove to be a very accurate tool for estimating plasma parameters and related phenomena.

\section{Acknowledgements}

This work is part of the research programme 'Controlling photon and plasma induced processes at EUV optical surfaces (CP3E)' of the 'Stichting voor Fundamenteel Onderzoek der Materie (FOM)', which is financially supported by the 'Nederlandse Organisatie voor Wetenschappelijk Onderzoek (NWO)'. The CP3E programme is co-financed by Carl Zeiss SMT and ASML. The authors also acknowledge support from the Province of Overijssel, PANalytical, DEMCON, Solmates, and the University of Twente through the programme of the Industrial Focus Group XUV Optics. 



\title{
Chapter 7
}

\section{Numerical and experimental studies of the carbon etching in EUV-induced plasma}

\begin{abstract}
We have used a combination of numerical modeling and experiments to study carbon etching in the presence of a hydrogen plasma. We model the evolution of a low density EUV-induced plasma during and after the EUV pulse to obtain the energy resolved ion fluxes from the plasma to the surface. By relating the computed ion fluxes to the experimentally observed etching rate at various pressures and ion energies, we show that at low pressure and energy, carbon etching is due to chemical sputtering, while at high pressure and energy a reactive ion etching process is likely to dominate.
\end{abstract}

\subsection{Introduction}

Many vacuum mirror optical tools suffer from the build-up of carbon contamination due to cracking of hydrocarbons under powerful vacuum ultraviolet radiation [122]. The large absorption of EUV radiation by carbon becomes significant in the case of multi-element optical systems, where throughput can be greatly reduced by even a very thin layer of carbon contamination on the top of each mirror.

The problem of EUV induced carbon contamination has been addressed in a series of publications $[123,10,124,89]$. The reduction of carbon films in a hydrogen atmosphere or hydrogen plasma has also been extensively studied [125, 126, 127, 128, 129]. Despite numerous studies, however, it is still difficult to predict the carbon removal rate, because there are many contributing factors. Several aspects that significantly affect the carbon removal rate include: many different allotropes and compounds of the carbon (e.g. soft black or hard graphite), many different contributing reaction paths (e.g. physical sputtering, chemical sputtering, reactive ion etching etc.), and, last but not least, small admixtures to the background gas, which, while residing on the carbon surface, can produce reactive species once irradiated by EUV. Despite this complexity, experiments have shown that carbon etching can be achieved under certain EUV-induced plasma conditions. Nevertheless, it has proven difficult to fully understand the etch process, because the characteristics of the EUV-induced plasma are poorly known, and the plasma-surface interaction has many contributing factors [129].

In this paper, we use a model of the EUV-induced plasma to numerically analyse the fluxes from the plasma to the sample surface. Our model is a self-consistent 2D Particle-in-Cell model of the weakly ionized low pressure hydrogen plasma that is formed during the EUV pulse due to ionization by EUV photons and secondary electrons from the surface. As described in Section 7.3, our model provides an accurate estimate the ion flux composition and energy distribution. However, a considerable number of parameters required for accurate simulations are not well known, 
therefore, the modeling results were combined with experimental observations. With the aid of simulations, we show that the shape of the energy distribution function of the ion fluxes in the considered experimental setup are mainly defined by the setup geometry, background pressure, and externally applied bias voltage. The ion dose, on the other hand is sensitive to the variations of many other parameters (e.g. EUV dose, secondary electron yield, etc.).

By combining the computed energy distribution function of the ion flux with the experimentally measured ion dose, insight into the mechanism for carbon removal was gained. By analysing the differences in yield between EUV-induced plasma and surface wave discharge plasma experiments in combination with numerical simulations, we show that chemical sputtering dominates for low pressures and energies. It was found that the carbon removal yield for both the surface wave discharge and EUV-induced plasmas was similar in the overlapping energy range. Hence, the effect of the EUV radiation on carbon removal is found to be significantly smaller than was estimated previously [129].

\subsection{Experimental setup}

The ISAN EUV experimental setup is based on a tin EUV radiation source, which is a Z-pinch discharge plasma with $1500 \mathrm{~Hz}$ repetition rate, which has been described in detail elsewhere [93]. This source is a good tool for exploring EUV-induced surface processes over a large number of pulses ( $>1 \mathrm{MShot}$ ). In brief, EUV radiation is introduced into a so-called "clean" chamber (see Fig. 7.1), separated from the source and collector optics by a Si:Zr spectral purity filter (SPF). The clean chamber is differentially pumped to pressure of $3.5 \times 10^{-8}$ torr. Under vacuum conditions, the background hydrocarbon carbon growth rate was measured to be $0.4 \pm 0.2 \mathrm{~nm} / 10$ Mshot.

The diameter of the EUV beam at the sample was $5 \mathrm{~mm}$. In addition to the direct beam, some scattered EUV radiation was also incident on the sample. The EUV pulse duration is about $100 \mathrm{~ns}$ (FWHM), with a tail, as described in [15].

The incident EUV power was measured using a sensitive thermo-couple attached to a thin copper disk. It was found that the radiation intensity was $0.13 \mathrm{~W} / \mathrm{cm}^{2}$ after the SPF and approximately $0.75 \mathrm{~W} / \mathrm{cm}^{2}$ without the SPF. The ratio of EUV intensities with and without the SPF corresponds to the calculated transmission of the $100 \mathrm{~nm} \mathrm{Si:Zr} \mathrm{SPF}$ filter over an energy range of 60 to $100 \mathrm{eV}$ (see Fig 7.2).

The EUV intensity on the sample decreases with time because of carbon growth on the SPF filter and focusing optics. In later experiments, the EUV intensity was measured to be $0.1 \mathrm{~W} / \mathrm{cm}^{2}$ after the SPF.

During the experiments, the hydrogen pressure was set in the range of $2.8 \mathrm{~Pa}-86.5 \mathrm{~Pa}$. To ensure that each radiation pulse excited a plasma in an atmosphere dominated by hydrogen, hydrogen flowed through a liquid nitrogen trap (to remove water) and into the chamber at 100 liters $\times$ torr/minute .

To control the energy of the ion flux, the sample holder assembly was biased in the range of $-200-0 \mathrm{~V}$, while all other metallic electrodes were grounded. The samples consisted of carbon, deposited by magnetron sputtering on a silicon wafer to a thickness of $\sim 30 \mathrm{~nm}$. Each sample was exposed to the $10^{7}$ EUV pulses at a different combination of bias and hydrogen pressure. After exposure, the amount of carbon removed was measured by X-Ray fluorescence (XRF) (EDS) 


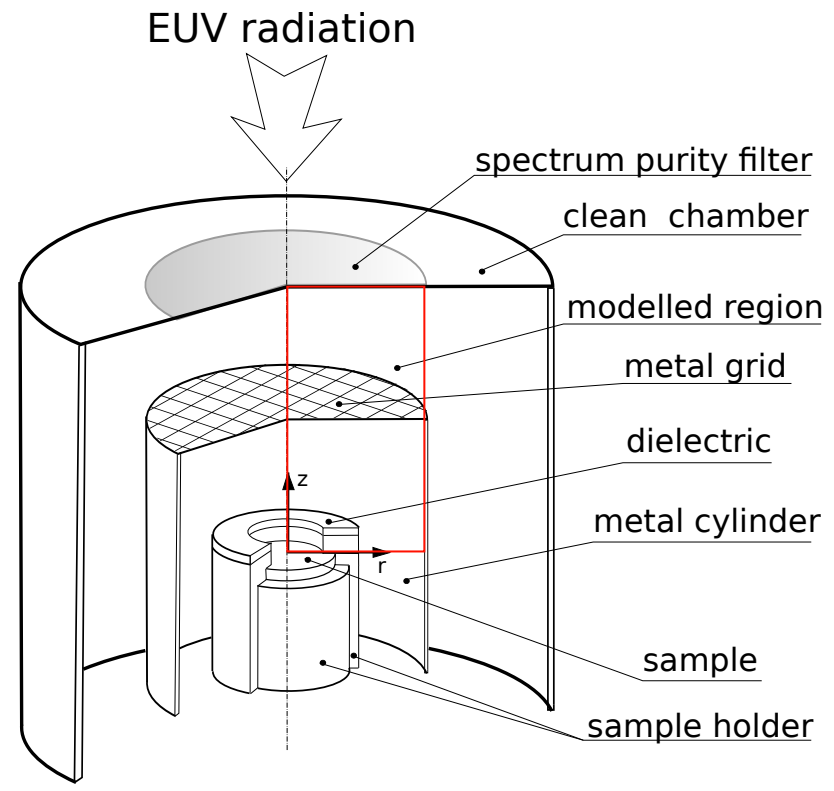

Figure 7.1: Configuration of the experimental chamber. The metal cylinder inner diameter is $28 \mathrm{~mm}$, the sample holder diameter is $18 \mathrm{~mm}$, the inner radius of dielectric mica diaphragm is $8 \mathrm{~mm}$, the distance from sample to grid is $2.4 \mathrm{~cm}$, distance between the grid and SPF is $1.5 \mathrm{~cm}$.

analysis and spectroscopic ellipsometry.

\subsection{Model}

A two dimensional particle in cell (PIC) model with $r z$ geometry was used to model the experiment. Our model follows the general PIC scheme, described elsewhere [28].

Ionization is initiated by an EUV pulse, which directly ionizes the background gas, and produces electrons by photo emission from the SPF and the sample. This process leads to the formation of an EUV induced plasma in the chamber. The ionization and photo-emission process and their inclusion in the model is described below.

Although the plasma is continually re-ignited by the pulsed EUV radiation, we consider each EUV pulse to induce a plasma in a cold neutral gas that is at equilibrium, because the characteristic time of plasma decay in the given geometry is about $\sim 20 \mu \mathrm{s}$, which is much shorter than the $660 \mu$ s between pulses. The restoration of thermal equilibrium in the background gas is also much shorter then the time between pulses.

\subsubsection{Chamber configuration}

The configuration of vacuum chamber is presented in Fig.7.1. Because the chamber is axially symmetric, it is possible to include the structure of the internal chamber in the simulations. To accurately model the plasma dynamics when the sample is biased, the space between the SPF 
and the metal grid is included in our simulations. The grid is included in the model as a number of metallic rings. When a bias is applied to the sample, ions from the plasma, which is formed between SPF and metal grid, are pulled towards the sample.

\subsubsection{Deielectric model}

In the experiment, a dielectric mica diaphragm was used to prevent EUV radiation being incident on the sample holder structure, thereby decreasing electron emission from the sample holder structure. Therefore, allowing plasma parameters to be estimated from the discharge characteristics of the sample. In the model, this feature is included with a simple dielectric model: a dielectric is not conductive, it can accumulate charge and the secondary electron yield under EUV irradiation is order of magnitude lower than for the sample.

The electron reflection and secondary electron emission under electron impact (SEE) are included in the model. These two processes are combined into SEE with the probability $(P(E))$ defined as follows.

$$
P(E)=p_{0}+\frac{E_{e}}{E_{1}}\left(1.0-p_{0}\right)
$$

Here $\mathrm{E}$ - is the energy of incoming electron. The parameters of the used mica are unknown, therefore, we choses $E_{1}=45 \mathrm{eV}$ and $p_{0}=0.7$, these parameters corresponds to linear fit for SEE yield for quarts in [130].

This approximation blends between significant slow electron backscattering for low energy electrons and secondary electron emission for higher impact energies. We do not use higher order approximations, since both effects are known to depend strongly on the surface conditions $[131,130]$.

This model for the dielectric is over simplified, but sufficient to allow us to to take the out of focus EUV radiation, which is incident on the diaphragm, into account in a consistent manner.

\subsubsection{Length scales and grid resolution}

The time evolution of one EUV pulse has two distinct stages: negative space-charge dominated during the beginning of the EUV pulse, and decay of the positively charged plasma after the pulse [22].

In order to model the negative space-charge dominated part of the plasma evolution, the potential well near the surface, which is formed by the electrons that have escaped to the volume, must be resolved. The length scale of the space-charge potential well depends on the energy distribution of photoelectrons from the surface and on the current which passes through the system.

For the purpose of estimation, it is possible to simplify the formulas from [74] and obtain

$$
z_{m} \simeq 0.1 \mathrm{~cm} \times \frac{(T[\mathrm{eV}])^{3 / 4}}{\left(I\left[\mathrm{~mA} / \mathrm{cm}^{2}\right]\right)^{1 / 2}}
$$

Here, $T$ is the initial temperature of the emitted photoelectrons, $I$ is the current density near the cathode, and $z_{m}$ is the distance from the cathode to the bottom of the space charge potential well. For $T \sim 1 \mathrm{eV}$ and $I \sim 20 \mathrm{~mA} / \mathrm{cm}^{2}$, one obtains $z_{m} \sim 0.02 \mathrm{~cm}$. 
For the plasma dominated part of the plasma evolution we need to resolve the Debye length in the volume, and also have a finer grid near the surface to resolve the plasma sheath.

From the EUV intensity $(\sim 0.02 \mathrm{~mJ}$ per pulse) and background hydrogen pressure (2.8 $\mathrm{Pa}$, $11.2 \mathrm{~Pa}$ ), the plasma density can be estimated to be about $10^{9} \mathrm{~cm}^{-3}$. For $T_{e} \sim 0.5 \mathrm{eV}$ and $N_{e} \sim$ $10^{9} 1 / \mathrm{cm}^{3}$ one obtains $R_{D} \sim 0.015 \mathrm{~cm}$, which is comparable to the space charge length scale estimated above. To achieve this, the grid is refined near the sample surface and SPF. The minimum grid cell size is $0.05 \times R_{D}$, and is gradually increased in steps of $5 \%$ until the bulk cell size of $0.5 \times R_{D}$ is reached. Several tests were performed to ensure that the chosen grid resolution does not affect the plasma dynamics.

\subsection{4 photo-electron emission}

The energy spectrum of photoelectrons emitted from the SPF and sample surface are calculated from [39]:

$$
P(E) \sim \frac{E}{(E+W)^{4}}
$$

Here $P(E)$ is the probability of emitting an electron with energy, $E$, from a surface with a work function, $W$. For carbon $W=5 \mathrm{eV}$ [40]. For electrons emitted from the surface, we assume an angular dependence given by a cosine emission law [39]. For purpose of estimation, we used the same approximation for the spectrum of photoelectrons emitted from the dielectric.

\subsubsection{EUV spectrum and photoionization}

To calculate the distribution of ion species, the spectrum of the EUV radiation must be included in the model, along with the energy-dependent cross sections for each photoionization process. Direct photoionisation of hydrogen by EUV photons is included as two processes:

$$
\begin{aligned}
& \mathrm{H}_{2}+h \nu \rightarrow \mathrm{H}_{2}^{+}+e \\
& \mathrm{H}_{2}+h \nu \rightarrow \mathrm{H}^{+}+\mathrm{H}+e
\end{aligned}
$$

The photoionisation cross-sections were taken from [36].

In the experiment, the EUV spectrum was measured (green curve Fig. 7.2) and found to be in agreement with data from [15]. The EUV spectrum, however, was measured before reflection from the Zr collector mirrors, and transmission through the SPF.

The calculated transparency curve that was provided with the Si:Zr SPF was used to calculate the EUV spectrum after transmission through the SPF (See Fig. 7.2 blue curve).

The SPF transmission curve has a transmission of about $1 \%$ in the range of $20-30 \mathrm{eV}$, which is important because the photoabsorption cross-section is very large in this energy range (see Fig. 7.2 red curve).

The contribution due to radiation in the $20-30 \mathrm{eV}$ range is difficult to quantify for two reasons: the intensity of the radiation varies significantly due to carbon growth on the SPF, and the accuracy of the SPF transmission curve in this energy range is unknown. Nevertheless, we include it because, even a small transmission will result in additional photoinization, which leads to a reduced space-charge potential barrier at low pressures (e.g. 2.8 $\mathrm{Pa}, 11.2 \mathrm{~Pa}$ ). 


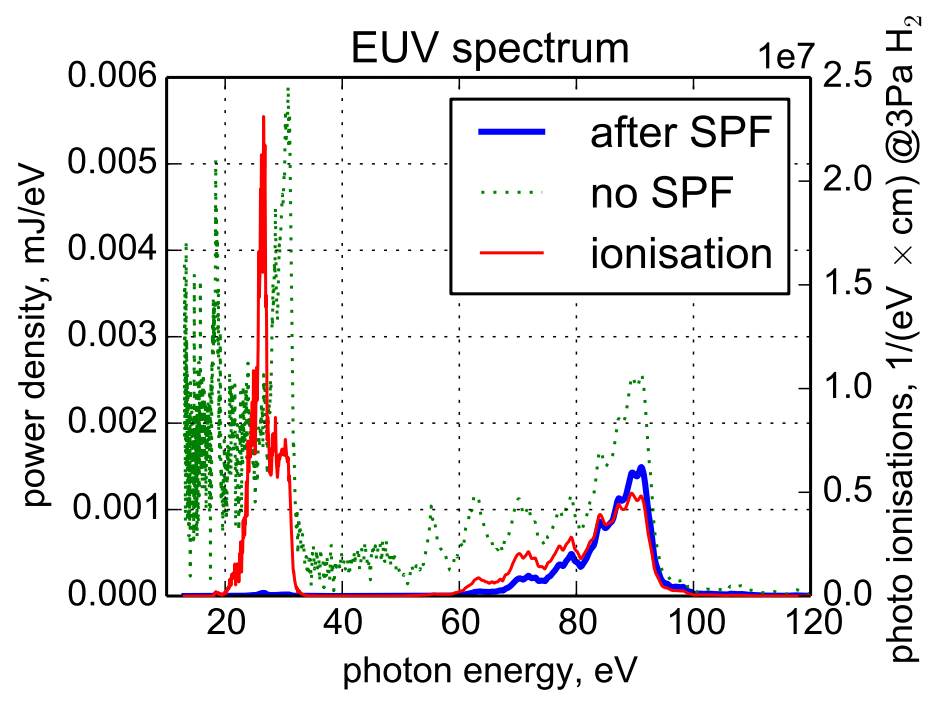

Figure 7.2: EUV spectrum after SPF as used in the simulations. Note, that the small SPF transmission, estimated at $\sim 1 \%$ of EUV energy per pulse, significantly contributes to the number of photoionisation events in the volume.

Although the photoionization due to the $20-30 \mathrm{eV}$ radiation range is significant compared to the $13 \mathrm{~nm}$ band, it has significance only for cases where no bias is applied to the sample. For cases with bias, the presence of this radiation leads to a decrease of the space-charge potential.

\subsubsection{Cross-sections set}

The cross-sections set used in the model consists of electron collisions with hydrogen and ion collisions with hydrogen. The collisions between plasma species and three body processes are neglected due to their low probability under the conditions considered here. To accurately model electron collision related processes in hydrogen discharges with a Monte Carlo (MC) model, one needs to take into account the differential cross-sections for ionisation and excitations processes. As described in detail in [64], the particular choice of the angular dependence of cross-sections significantly influences the simulation results. We adopt a set of cross sections found in Ref. [64].

We use an approach similar to that described in [64]. The reaction probability is sampled using the integrated reaction cross-section. The differential cross-section data is used to determine the collision kinematics and energy redistribution between products. Electron elastic scattering and hydrogen electronic excitations, and angular scattering data is taken from [46]. For electron impact ionization of hydrogen, we use an experimentally determined doubly differential crosssection [57, 58]. The set of cross-sections for collisions between ions and hydrogen is based on [62] because this set provides good agreement with swarm data for ions in hydrogen. We neglect the formation of $\mathrm{H}^{-}$, because the cross-section of dissociative electron attachment is very low, and the density of vibrationally exited hydrogen molecules too low to make a significant contribution to the production of $\mathrm{H}^{-}$. 
We make use of the procedure described in [42] to perform Monte Carlo collisions with the background gas. We tested the consistency of our implementation by modeling swarm experiments and found good agreement with experimental values [54] for the first Townsend electron ionization coefficient, the electron mobility, and for $\mathrm{H}^{+}$and $\mathrm{H}_{3}^{+}$mobility in hydrogen [55].

\subsection{Analysis of the charge - bias characteristic}

Let us begin with the analysis of the charge - bias voltage characteristics $(Q-V)$, which were measured during the experiments. The ion dose on the sample per pulse for the range of pressures and bias voltages considered here was estimated from the experimental results as a difference between the collected charge for given pressure and bias voltage and the collected charge for vacuum conditions [129]. The measured curves are presented in Fig.7.3. It is worth noting that these $Q-V$ characteristics have some peculiarities.

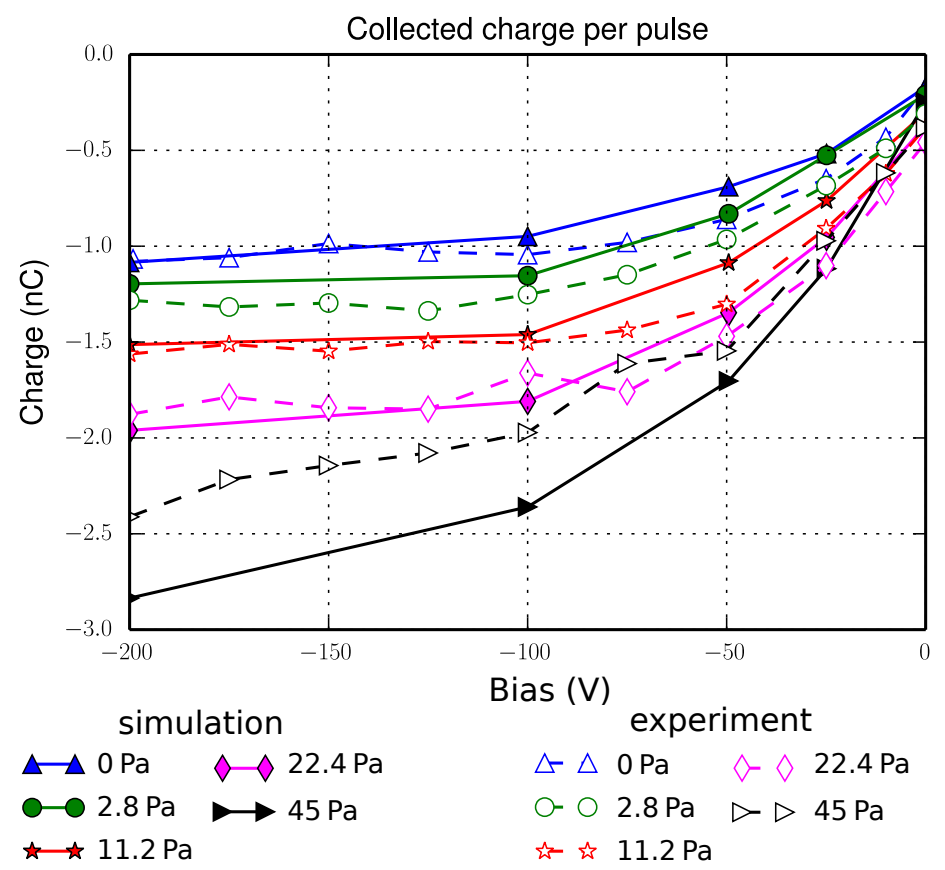

Figure 7.3: Collected charge as function of bias, $0.017 \mathrm{~mJ} /$ pulse $\left(0.13 \mathrm{~W} / \mathrm{cm}^{2}\right.$ at $1500 \mathrm{~Hz}$ to the spot of $5 \mathrm{~mm}$ diameter.

Firstly, all the characteristics reach saturation, and the turning point for the almost all curves is approximately $-75 \mathrm{~V}$. If there is a significant contribution from ionization by the accelerated secondary electrons from the sample in the drift regime, one would expect a rapid increase of the collected charge for biases in the range of -200 .. $-50 \mathrm{~V}$.

Secondly, the increase of the collected charge for $-200 \mathrm{~V}$ bias does not depend linearly on pressure in the range of $2.8 \mathrm{~Pa}$ to $22.4 \mathrm{~Pa}$, i.e. the collected charge increases less than two-fold while the pressure increases eight times. But, one would expect at least linear growth of the 
collected charge with pressure if the volume ionization provides a significant contribution to the total collected charge. Thus, the overall contribution of photoionization in the volume is small compared with the other factors.

Thirdly, for -200 V bias and 22.4 Pa background pressure, due to the photoionization process, all the external bias would be applied over a very small layer near the sample, thus, all the secondary electrons will gain $200 \mathrm{eV}$ energy. The $\mathrm{H}_{2}$ ionization cross-section for this energy is approximately $6 \cdot 10^{-17} \mathrm{~cm}^{2}$, thus, in the space between the sample and the grid $(l \sim 2.5 \mathrm{~cm})$, these electrons will, on average, have 0.8 ionization inducing collisions. If we assume that, under vacuum conditions and $-200 \mathrm{~V}$ bias, the SE current from the sample saturates, and we obtain that, just due to the direct electron induced ionization, without cascade process and photoionization, the collected charge should be greater than $1.8 \mathrm{nC}$. in the experiment, however, the collected charge is approximately $1.9 \mathrm{nC}$.

\subsubsection{Average secondary electron yield}

The above analysis suggests that the combination of EUV power per pulse, and effective secondary electron yield (SEY) produces approximately $1 \mathrm{nC}$ of electrons from the sample.

It is instructive to estimate the total SE charge from the experimental parameters. From the EUV intensity, repetition rate and spot size, the average dose per pulse was approximately $\sim 0.017 \mathrm{~mJ}$. The secondary electron yield for carbon under EUV radiation is estimated to be approximately $\gamma_{S E} \sim 0.01$ for the photon energy range of $60 \mathrm{eV}-100 \mathrm{eV}$ [37, 123], which leads to a saturation charge of $2 \mathrm{nC}$, which would lead to a significant disagreement between the simulated and measured charge bias characteristics. It is also worth noting that effective SEY from mica was small, since, in the experiment, the charge collected from a sample made from mica was measured to be an order of magnitude smaller than for the carbon sample.

However, the measured charge bias characteristics are the only experimental data that provides a reference point for the simulations of the experiment dynamics. Since the spatial distribution of EUV intensity is subject to an unknown systematic measurement error, and the SEY is known to vary widely, depending on the surface conditions, we chose to keep the product of incident EUV and SEY a constant, chosen to provide $1 \mathrm{nC}$ total SE charge from the sample.

\subsubsection{Role of dielectric ring}

During the simulations we found that the mica accumulated charge over many EUV pulses, significantly effecting the local field distribution, and, hence changing the flux incident on the sample. If charging is neglected, the $\mathrm{Q}-\mathrm{V}$ response under vacuum and $2.8 \mathrm{~Pa}$ conditions cannot be reproduced for any reasonable parameter values. The discrepancy is caused by the potential barrier near the sample surface, which is created by the negative space charge generated during the EUV pulse. Although the potential is low, it produces a significant effect because the SE energy spectrum (7.3) is strongly peaked at rather low energies: approximately $W / 3=1.3 \mathrm{eV}$.

The charge accumulation on the mica significantly increases the local field strength near the sample surface (see Fig. 7.4). This removes the space charge potential barrier for biases of $-100 \mathrm{~V}$ bias and higher under the conditions that we consider. 


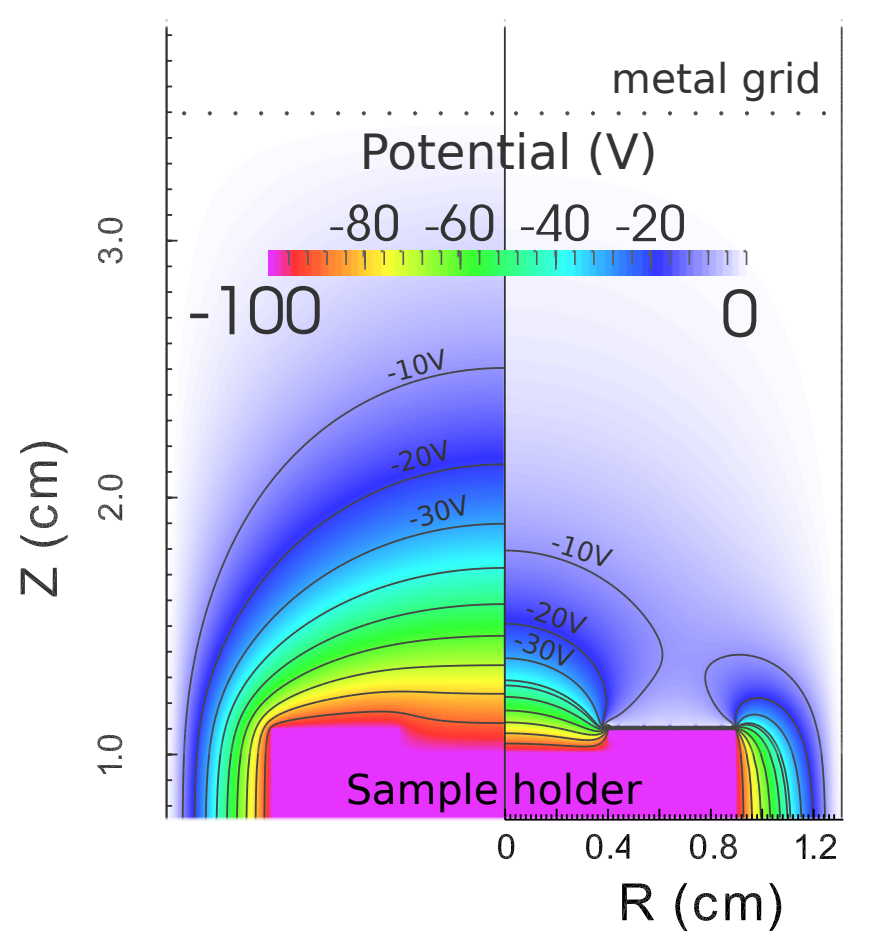

Figure 7.4: Comparison of the potential distibution hear the sample holder for uncharged mica (left) and pre-charged mica for $-100 \mathrm{~V}$ bias on the sample holder. The charge on the mica significantly increases the local field strength near the smaple surface.

The charging of the mica should saturate, leading to an unchanging charge density distribution on the mica. To estimate the charge density, we simulated one hundred pulses under vacuum conditions for all experimentally applied bias values. In these calculations, the effective SEY from the mica is assumed to be about 0.001 , which corresponds to the experimentally measured value.

This approach corresponds to the experimental procedure, since the $\mathrm{Q}-\mathrm{V}$ characteristics were measured via averaging a large number of pulses for every combination of bias and pressure. No special means were used in the experiment to remove the accumulated charge from the mica between pulses.

For negative biases, simulations show that the mica potential rapidly reaches approximately $0 \mathrm{~V}$ potential. For these conditions, further charging is very slow, because (a) the electric field strength near the mica is very small, therefore, even a small amount of emitted charge creates a space charge potential barrier, and, (b), because the mica potential is close to zero, there is a small flux of electrons from the sample to the mica.

The continued slow variation in charge distribution is impractical to simulate, however. The final charge distribution requires a very long time to calculate and is sensitive to the combination of the SEY from both the sample and the mica, and to the corresponding SE energy distribution functions. Nevertheless, the additional errors due to these limitations are expected to be small. 
In practice the maximum potential of the mica under vacuum conditions is bounded, since, as the mica's potential increases to $\sim 10 \mathrm{~V}$, the electron current from the sample to mica becomes significant.

It is worth noting that the EUV plasma itself can contribute to charging the mica, since, for stationary discharges, the dielectric charges to the plasma potential. Despite the the fact that the potential of the EUV-induced plasma can be high (e.g. $20-30 \mathrm{~V}$ or more after EUV pulse), it rapidly decreases to several volts due to electron cooling due to collisions with the background gas. Most probably, the mica potential would converge to the time averaged potential of the plasma, e.g. several volts. Therefore, in the following results, the mica was pre-charged to the value that was obtained from simulations under vacuum conditions for the appropriate bias.

The optimum parameter combination that reproduces the experimentally measured $\mathrm{Q}-\mathrm{V}$ characteristics were chosen as follows: the mica was pre-charged as described above, the EUV intensity was kept constant at the measured value, but the amount of the scattered EUV radiation incident on the sample holder was chosen so that $40 \%$ of EUV radiation was incident on the dielectric mica, and the effective SEY from the sample was kept at to 0.01 for the photon energy range of $60 \mathrm{eV}-100 \mathrm{eV}$, while the SE contribution from the VUV part of the spectrum was neglected. The simulated $\mathrm{Q}-\mathrm{V}$ characteristic, after parameter optimization, and comparison with the measurements are presented in Fig. 7.3.

\subsection{Ion fluxes to the sample surface}

The removal of carbon from the surface material should be directly dependent on the energy distribution function (EDF) of the ion flux incident on the surface. The conditions of the experimental study of carbon cleaning in the ISAN EUV experiment are summarized in Table 7.1. We computed the plasma conditions, ion fluxes, and ion EDF for these conditions. The simulated time for all cases was $10 \mu \mathrm{s}$. For $2.8 \mathrm{~Pa}-11.2 \mathrm{~Pa}$ and biases $-200 \mathrm{~V}--100 \mathrm{~V}$, the plasma had completely decayed and all ions were collected during the simulation time. But, for the $60 \mathrm{~Pa}$ and 86.5 Pa cases some plasma was still left in the simulation domain.

The EDFs, integrated over the simulation window, and averaged over the sample surface, are presented in Fig. 7.5. The composition of the computed ion flux depends significantly on the pressure. For the $3 \mathrm{~Pa}$ case, the main ion is $\mathrm{H}_{2}^{+}$, because the characteristic time for $\mathrm{H}_{2}^{+}$to $\mathrm{H}_{3}^{+}$ conversion in $3 \mathrm{~Pa} \mathrm{H}_{2}$ is approximately $0.5 \mu \mathrm{s}$. With increasing pressure, $\mathrm{H}_{3}^{+}$becomes the main ion, as expected. There is also a non-negligible contribution from fast $\mathrm{H}_{2}$ which is produced due to a resonant charge exchange reaction.

The maximum ion energy is larger than the applied bias voltage due to the build-up of the plasma potential. But the number of such fast ions is small, due to the fast plasma decay. For the same reason, the most energetic ion is $\mathrm{H}^{+}$because its small mass allows it to accelerate during the decay of the plasma potential.

It was observed, that contrary to the ion dose, the shape of the ion flux EDF was mainly defined by bias, pressure and the accumulated charge on the dielectric. However, as discussed in previous section, we expect that the equilibrium mica potential does not significantly differ from 0 V. For small variations of the mica potential (if all other parameters are the same), the variations of the EDF shape are small for all simulations where a sample bias was applied. Moreover, the 

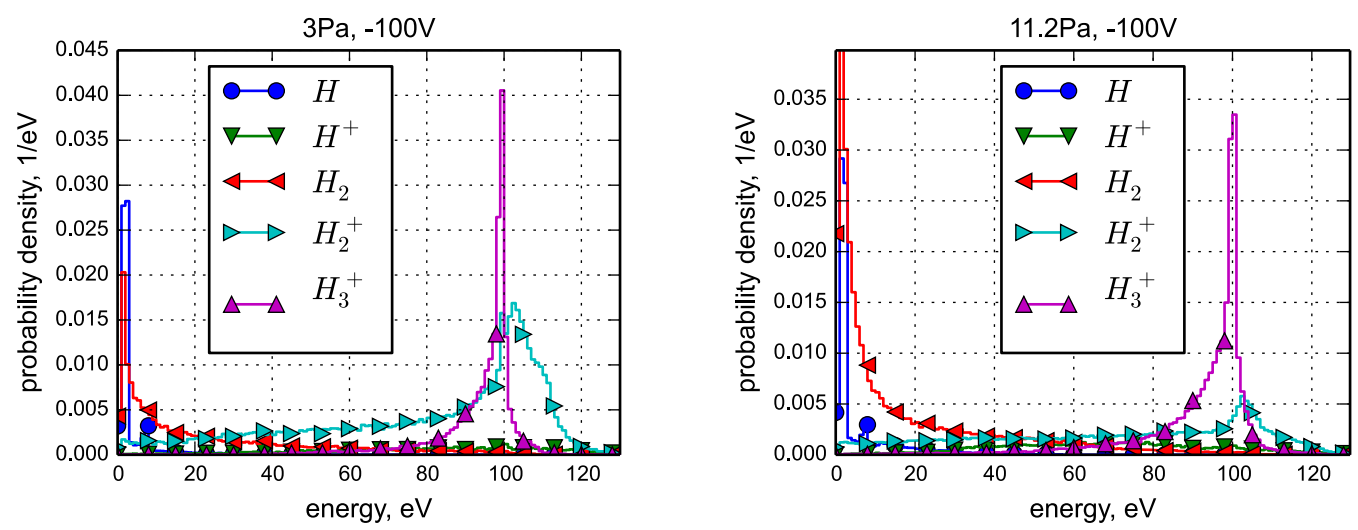

Figure 7.5: Energy distribution function of ion and fast neutrals flux to the surface for $3 \mathrm{~Pa}$ (left) and 11.2 $\mathrm{Pa}$ (right) for $-100 \mathrm{~V}$ bias. The fluxes were integrated over time and over sample surface and sum of fluxes normalized to unity. Note that $\mathrm{H}_{3}^{+}$spike is on the same position over energy in spite of the increase of pressure.

EDF was barely sensitive to reasonable variations of other parameters.

This insensitivity is a consequence of the experimental procedure. The applied bias allows ions to be collected from the entire chamber volume. Hence, the characteristics of the plasma are important only for the short time when the sheath between plasma and sample is small. As ions are collected at the sample, the sheath size becomes larger and larger. Therefore, as time progresses, the instantantaneous ion flux EDF starts to depend only on the bias potential, mica potential, and the distance that the ions travel between the plasma and the sample. This is because these parameters determine the maximum possible energy of the ions, while the background pressure determines the number of collisions (e.g energy loss), and, hence, the average energy of the ion at the sample. Therefore, for the considered conditions, the shape of the ion flux EDF is mostly determined by the bias, mica potential, pressure, and the chamber geometry.

It is notable, that the mica charge, due to exposure to EUV radiation and plasma, leads to the ion flux being focused on the sample (see Fig. 7.7). The ion flux to the mica itself becomes negligible compared to the simulation with zero charge on the mica. Therefore, the measured ion doses should represent accurately the ion doses on the sample.

Hence, for the analysis of the experiments, it is reasonable to take the computed EDF shape, normed to the experimentally measured ion dose (if it is available). This approach allows us to compare the experimental results at different pressures.

\subsection{Discussion}

The maximum etched depths and total etched volumes per exposure of $10^{7}$ pulses for different pressures and biases are presented in Table. 7.1. The etched volumes are the integrals over etch profiles, which were determined by XRF measurement (see Fig. 7.8). In the cases of $60 \mathrm{~Pa}$ and 86.5 Pa pressure, the etch profile was only measured at the center of the EUV spot. We assumed, 


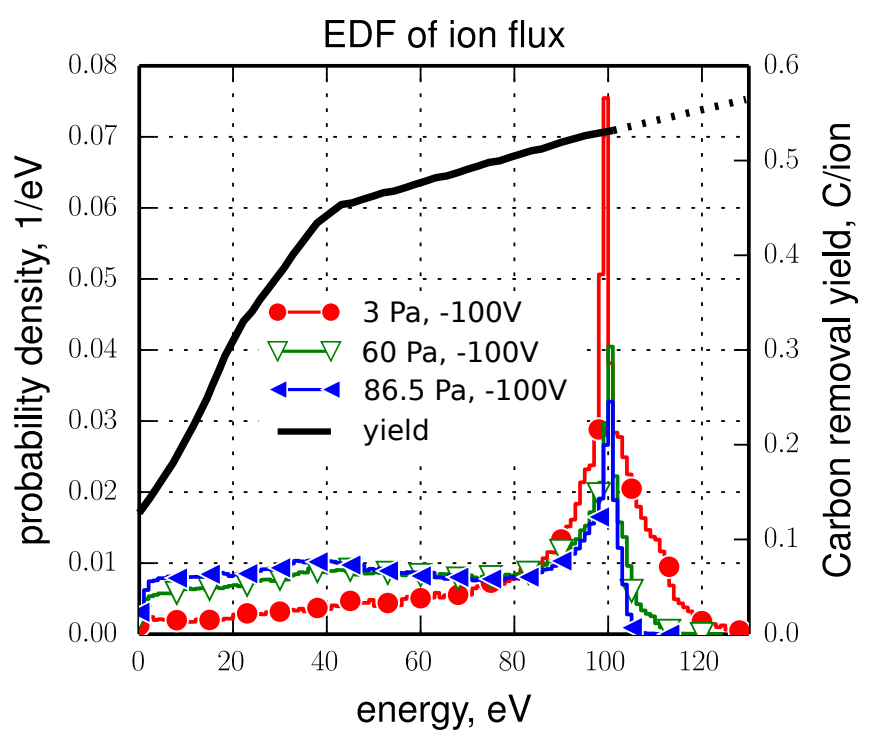

Figure 7.6: Comparison of the shape of the ion flux EDF obtained with pre-charged dielectric mica for different pressures. The ion fluxes were integrated over time and over sample surface, summed over ion types and normalized to unity. The carbon removal yield curve corresponds to surface wave discharge plasma (SWD) from [129]. The dotted part of the yield curve corresponds to linear extrapolation.

for these two cases, a uniform etching profile to estimate the carbon removal yield. Hence, for these cases, the amount of removed carbon is most probably overestimated.

As was discussed in the previous section, we used the experimentally measured ion doses for yield estimation. However, for some experiments, the ion dose was not measured. In these cases, we used the ion doses obtained from simulation results. This approach is valid at high pressures because the uncertainty in the simulations is on the order of $30 \%$ (see Fig. 7.3).

For all samples, the average carbon removal yield was larger than 0.2 carbon atoms per ion. Thus, physical sputtering is not the main process, since for energies below $200 \mathrm{eV}$, the expected sputtering yield is lower than $5 \cdot 10^{-2}$ carbon atoms per hydrogen ion [132]. Therefore, carbon is removed via a chemical sputtering process, or a reactive ion etching process, because the effective yield for these processes is known to be large under certain conditions [125].

However, the results for $-200 \mathrm{~V}$ bias, especially at low pressure $(2.8-11.2 \mathrm{~Pa})$, cannot be described by a chemical sputtering process, since the average yield is larger than one carbon atom per ion. Hence, reactive ion etching may be responsible for these very high etch rates. However, for reactive ion etching there should be weakly bound radicals on the top of the carbon, which are desorbed from surface due to ion impact, which, in turn, lead to a very high yield per ion. Although, we have no data about these radicals, it is likely to be some form of methane radical (e.g., $\mathrm{CH}_{x}$ ). The alternative: oxidation (e.g., $\mathrm{CO}$ and $\mathrm{CO}_{2}$ ) [123] is unlikely because water, which is the main source of oxygen, is removed from the hydrogen flow, and, during experiments, the 


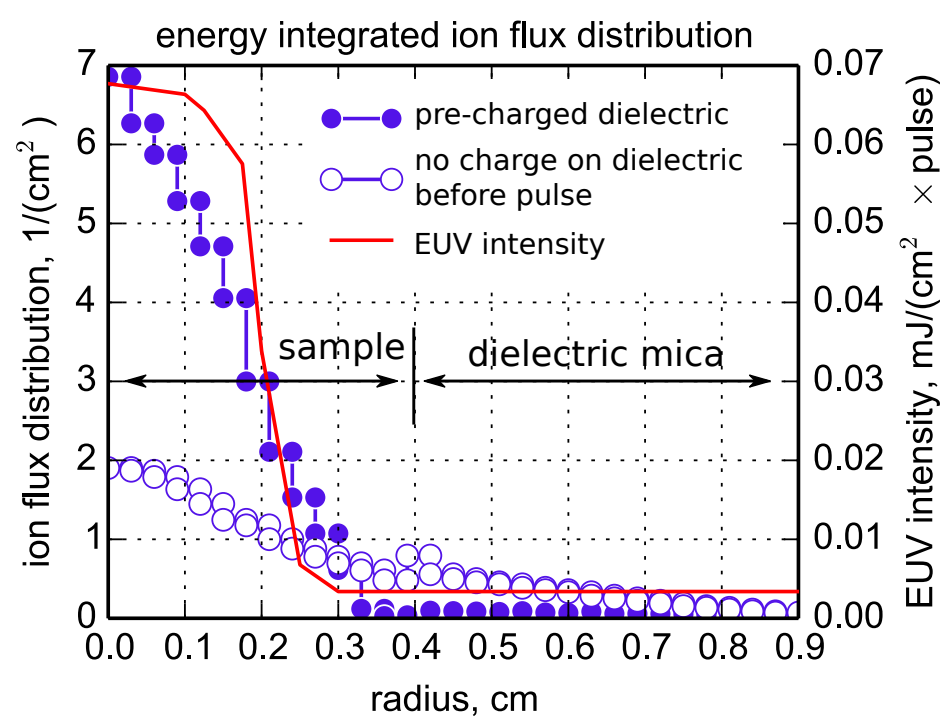

Figure 7.7: Comparison of the ion flux spatial distribution obtained with pre-charged mica and with zero charge on the mica. The ion fluxes were integrated over time and energy. Both distribution are normed to one if integrated over sample and dielectric mica surface areas. The conditions are $3 \mathrm{~Pa}$ background pressure and $-100 \mathrm{~V}$ bias on the sample. The charge on the mica focuses the ion flux on the sample. Other combinations of pressure and bias show similar behavior.

chamber walls are kept at liquid nitrogen temperatures, trapping the majority of the residual water. Hence, the contribution of water to the carbon removal was limited by the initial coverage which is typically sub mono layer for amorphous carbon for the considered temperature range [133].

In the case of experiments with $-50 \mathrm{~V}$ and $-100 \mathrm{~V}$ biases, the results can be compared with carbon etching in a surface wave discharge plasma [129], where similar magnetron deposited carbon on silicon substrate samples were used. The carbon removal yield for experiments with biases of $-50 \mathrm{~V}$ and $-100 \mathrm{~V}$ was estimated by convolving the simulated EDF with the energy dependent yield, as measured in the SWD experiments (see Fig. 7.6 and column "SWD recomputed" in table 7.1). In spite of $\mathrm{H}_{3}^{+}$being the main ion in SWD plasma, we used the same yield for estimation, since we do not expect dramatic difference between $\mathrm{H}_{3}^{+}$and $\mathrm{H}_{2}^{+}$for carbon removal. These estimates show that the yields found in EUV experiments with low biases agree with the recomputed yield from the SWD experiment. The margin of error is, however, large enough that we cannot completely exclude direct influence from EUV radiation.

\subsection{Conclusion}

The evolution of an EUV induced hydrogen plasma was simulated. The simulations, due to their close coupling to experimental conditions, allowed the magnitude, composition, and energy spectrum of the flux from the plasma to the surface to be estimated. Our model successfully 

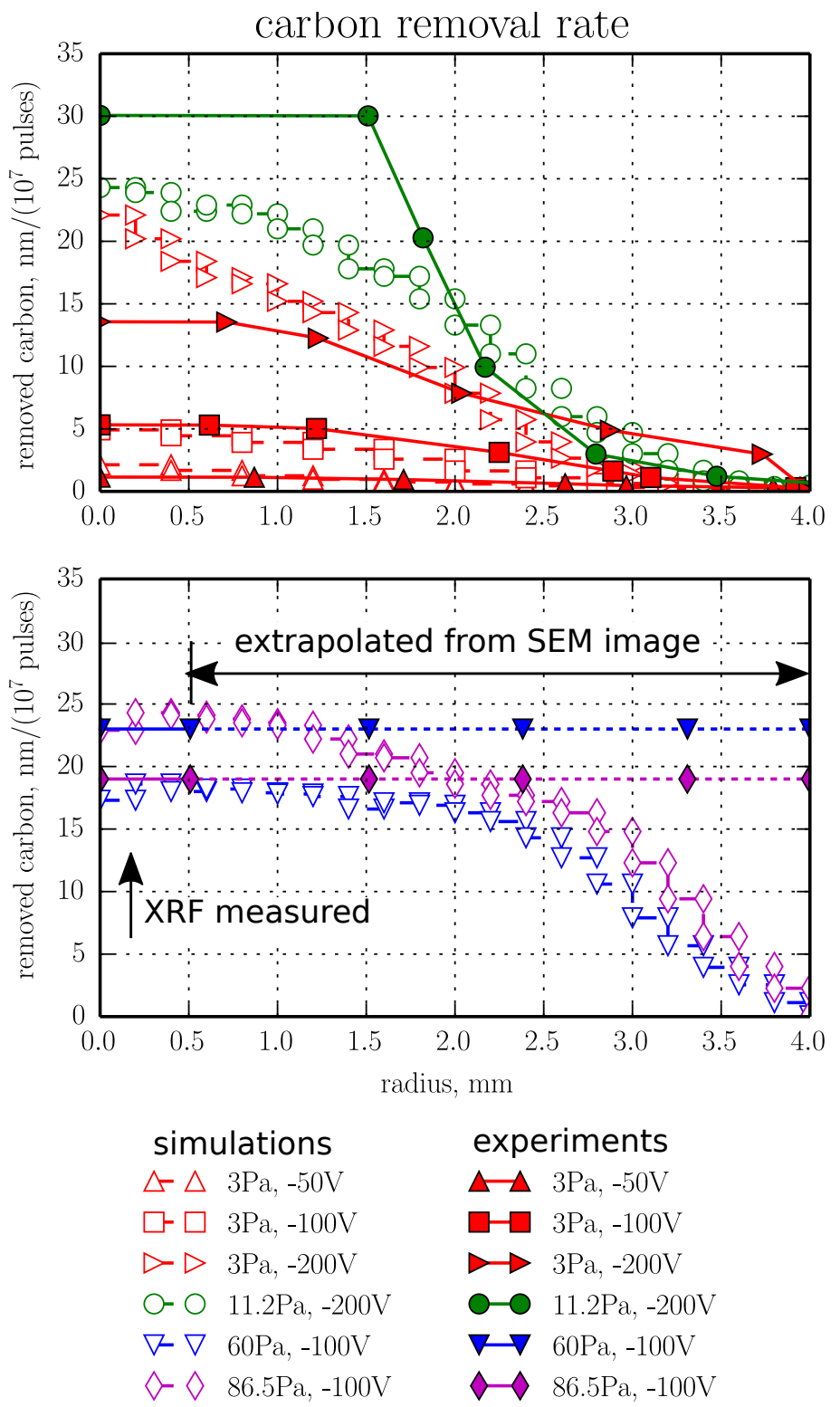

Figure 7.8: Experimentally observed and simulated carbon removal as function of pressure, bias and radius. For the conditions of $11.2 \mathrm{~Pa},-200 \mathrm{~V}$ the carbon layer was completely removed, exposing the substrate, at the center of the EUV spot. The etch yield for this case was set to the 1.6 $\mathrm{C}$ atom/ion as in table 7.1. For the conditions of $60 \mathrm{~Pa},-200 \mathrm{~V}$, the carbon layer was completely removed from entire area exposed to plasma, hence the case is not shown. The simulated profiles for $-50 \mathrm{~V}$ and $-100 \mathrm{~V}$ bias were computed via convolution of the simulated ions fluxes with the yield from SWD experiments Fig. 7.6. The profiles were normed to the ion dose per pulse from table 7.1. The EUV dose was $10^{7}$ pulses per sample in all cases, which is approximately $170 \mathrm{~J}$. The EUV radiation was concentrated in a spot with radius approximately $2 \mathrm{~mm}$. The detailed intensity distribution of the EUV spot is not accurately known. 
Table 7.1: Experimental conditions and carbon removal rate

\begin{tabular}{|c|c|c|c|c|c|c|c|}
\hline \multirow[b]{2}{*}{$\begin{array}{l}\text { pressure } \\
\mathrm{Pa}\end{array}$} & \multirow[b]{2}{*}{$\begin{array}{l}\text { bias } \\
\mathrm{V}\end{array}$} & \multirow[b]{2}{*}{$\begin{array}{l}\text { EUV power } \\
\mathrm{W} / \mathrm{cm}^{2}\end{array}$} & \multicolumn{2}{|c|}{ removed carbon } & \multirow{2}{*}{$\begin{array}{l}\text { ion } \operatorname{dose}^{b)} \\
\mathrm{nC} / \text { pulse }\end{array}$} & \multicolumn{2}{|c|}{ average yield } \\
\hline & & & $\begin{array}{l}\text { max etched depth } \\
\mathrm{nm} / 10^{7} \text { pulse }\end{array}$ & $\begin{array}{c}\text { etched volume } \\
10^{-6} \mathrm{~cm}^{3} / 10^{7} \text { pulse }\end{array}$ & & $\begin{array}{l}\text { present study } \\
\mathrm{C} \text { atom/ion }\end{array}$ & $\begin{array}{c}\text { SWD recomputed } \\
\mathrm{C} \text { atom/ion }\end{array}$ \\
\hline 3.0 & -50 & 0.11 & $1.7 \pm 0.5$ & $\sim 0.03$ & $\sim 0.1$ & 0.5 & 0.4 \\
\hline 3.0 & -100 & 0.11 & $5.7 \pm 0.5$ & $\sim 0.1$ & $\sim 0.25$ & 0.6 & 0.5 \\
\hline 3.0 & -200 & 0.13 & $14 \pm 0.5$ & $\sim 0.3$ & $\sim 0.25$ & 1.9 & - \\
\hline 11.2 & -200 & 0.13 & $30^{a)} \pm 0.5$ & $\sim 0.5$ & $\sim 0.5$ & $>1.6$ & - \\
\hline 60 & -100 & 0.13 & $23 \pm 0.5$ & $\sim 1.2^{c)}$ & $>2.2^{d)}$ & $<0.9$ & 0.5 \\
\hline 60 & -200 & 0.1 & $27 \pm 0.5^{e)}$ & $\sim 1.4^{c)}$ & $>2.3^{d)}$ & $\sim 1.0^{e)}$ & - \\
\hline 86.5 & -100 & 0.2 & $19 \pm 0.5$ & $\sim 1^{c)}$ & $>3.0^{d)}$ & $<0.5$ & 0.4 \\
\hline
\end{tabular}

a) The substrate was exposed, thus only lower boundary for cleaning rate could be estimated.

b) Estimated from measured Q - V characteristics (see Fig. 7.3) as the difference between the collected charge for a given pressure and bias voltage, and the collected charge for vacuum conditions.

c) The carbon removal was measured only in the center of the exposed area. However, an SEM image of the EUV exposed area showed no features. Therefore, for the purposes of estimating the carbon removal rate, we assume that the carbon was etched uniformly over the exposed area of the sample.

d) Experimental $\mathrm{Q}-\mathrm{V}$ values were missing, therefore the simulated ion dose were used for estimation. Only ions which hit the sample during the simulated period are included. No corrections were made for the plasma which was present in the chamber after the end of the simulation.

e) This value is only an order of magnitude estimation, because all carbon was removed from the sample.

computes the charge bias characteristics of the sample to an accuracy of a factor of two. This was used to quantitatively compare carbon etch rates between different EUV plasma conditions, as well as compare carbon etching under EUV-induced plasma to etching under SWD plasmas. In addition, the model describes the focusing effect of the dielectric surround, which allows the measured carbon etch profiles to be better understood in a quantitative manner.

It was observed that the carbon etching mechanism at low bias and pressure was different than that at high bias and pressure. For the higher energy range, the carbon removal yield in EUV-induced plasma was larger than one carbon atom per ion. Most probably, etching is dominated by a reactive ion etching process, which may be due to the production of methane radicals (e.g. $\mathrm{CH}_{x}$ ) that can desorb under energetic ion flux.

However, at low bias energies, the etch rates found in EUV-induced plasma agree with those found in SWD plasma to within model and measurement uncertainties. Therefore, the role of the EUV radiation in a previous study [129] was overestimated at low bias energies and pressures.

However, our study does not exclude EUV enhancing etch rates at low biases or having a small $(<0.2 \mathrm{C} /$ ion) enhancing effect over the range of biases and pressures that we studied. Therefore, experiments to determine the effect of EUV in the limit of low-to-no bias are required. 


\section{Acknowledgements}

The authors would like to thank Tatiana Rakhimova (Skobeltsyn Institute of Nuclear Physics) for helpful discussion.

This work is part of the research program "Controlling photon and plasma induced processes at EUV optical surfaces (CP3E)" of the "Stichting voor Fundamenteel Onderzoek der Materie (FOM)" which is financially supported by the Nederlandse Organisatie voor Wetenschappelijk Onderzoek (NWO). The CP3E programme is cofinanced by industrial partners, including ASML (Veldhoven), and the AgentschapNL through the Catrene EXEPT program. 


\section{Chapter 8}

\section{Valorisation and outline}

The design of the program developed in this thesis was determined by the industrial needs outlined in the chapter 1.1. However, from a more general plasma physics perceptive, it reduces to the need for a particle-in-cell code that can accurately and efficiently model low pressure low density transient plasmas. The code should include a volumetric source of ions and electrons that mimics the absorption of EUV radiation a long a path length of neutral gas.

The previous chapters of the thesis can be viewed as a successful validation of the model. We were able to reproduce, with good accuracy, measurements of currents in the EUV induced plasma in the ISAN setup (chapter 7). Therefore, the model can be straight forwardly applied to calculate ion fluxes from the EUV-induced plasma to MLM mirrors in EUVL applications.

It is worth to admit, that the for EUV induced plasmas both experiments and simulations tend to provide self consistent but misleading picture. For example the initial analysis of the experiments for carbon removal under EUV radiation pointed to the significant role of the EUV itself [129]. However, later analysis with help of the model showed that these conclusions were wrong. The cause was the wrong assumption about the uniformity of the ion flux over the sample surface. The simulations allowed to find that the ion flux was concentrated towards the center of the sample by the electric field (chapter 7). In the new analysis the role of EUV was estimated to be almost negligible.

The main benefit of simulations is the ability to make an quantitative estimation of the contribution of the different factors. In the typical experiments where are many of them some of them are well defined (e.g. chamber geometry), but most of them are poorly known EUV spectrum, EUV intensity, secondary electron yields from different materials. In most of experiments the discharge currents are recorded in order to trace the plasma. However, without the model only limited information of the data can be extract from such data because of two many contribution factors. It also worth to admit, that even for the Langmuir probes, that responses were profoundly studied for almost 100 years, the analysis of the probe response is still difficult, ad require certain care (see section 5 ).

Although, the large number of parameters is also a problem for simulations, the model can take into account the most probable range of variations of these parameters. Therefore, the model is viable numerical tool for analysis of the experiments.

\subsection{Outlook and applications beyond EUV-induced plasmas}

Apart from the application described above, plasma models have high relevance for many other industrial and scientific applications. However, the present code can not be easily applied outside of the low pressure $(1-30 \mathrm{~Pa})$ low density $\left(10^{7}-5 \cdot 10^{9} 1 / \mathrm{cm}^{3}\right)$ laboratory plasma regime. Below, 
we outline a few typical applications for plasma modeling and some of the modifications that would be required before the model presented here would be suitable for various applications

- Simulations of the EUV plasma induced by a free-electron laser (FEL) based radiation sources. The FELS are considered for application in EUVL [134]. However, their characteristics differ significantly in their pulse repetition rate, temporal duration, spectral content, and spatial and temporal coherence from plasma based sources, e.g., while a plasma based EUV source has a pulse duration of about $100 \mathrm{~ns}$ and a repetition rate in the range of $1 \mathrm{kHz}$ (DPP) .. $100 \mathrm{kHz}$ (LPP), a FEL based source has pulse width of about $10^{-5} . .10^{-6}$ ns (i.e. $10 . .100 \mathrm{fs}$ ) and a repetition rate of about $10^{5} \mathrm{kHz}$. Moreover, the FEL pulse duration is comparable to the characteristic electron relaxation time in a solid and is expected to be much shorter than the inverse plasma frequency of the plasma generated in the background gas. Therefore, in comparing plasma based EUV sources and FEL radiation, the main differences are expected to arise from the interaction of the radiation with the MLM, where photoelectrons and secondary electrons are generated. It might be expected, for instance, that simulations of an EUV plasma, ignited by a femtosecond FEL pulse, requires a different description of the photo electron emission from the surface compared to the case for nanosecond pulses.

- EUV palsmas. One limitation of the code, as it is currently implemented, is that each EUV pulse is considered to generate a plasma in a cold neutral gas. However, the repetition rates in the EUVL sources are expected to be large. In this case, the background can also be expected to contain a low density cold plasma as well. Therefore, the model should be extended to include a fluid model to accommodate the accumulation and flow of this background plasma.

- Material processing (e.g. etching). The model could be modified to be able to calculate capacitively coupled plasma discharge. For a small plasma density $\left(<10^{9} 1 / \mathrm{cm}^{3}\right)$, it is enough to extended the model with a submodule to iterate over periods of the radiofrequency field. Such a submodule allows a steady state solution to be found. However, industrial plasma etching equipment use plasmas with densities of about $10^{10}-10^{11} 1 / \mathrm{cm}^{3}$ or even higher. The efficient usage of PIC code for these plasmas would require the Poisson equation to be changed to the Poisson-Boltzmann equation, where the motion of the electrons is solved together with the electric field. This would require significant rework of the code. Moreover, for these kind of plasmas the constant background assumption is violated. Therefore, the code should be modified to take into account the production and accumulation of neutral species.

- Radiation sources (e.g. lamps). These plasmas have the similar problems as discussed for others. However, plasmas designed for radiation emission should include radiation transport, and step wise excitation/ionization processes are significant. Hence, these processes should be implemented in the model in order to compute these kind of plasmas.

- Space relevant plasmas. These can be split into:

- Space craft interaction with plasma, e.g. charging under space radiation. These application require a significantly more sophisticated implementation of the plasma 
surface interaction. More specifically, the generation of secondary electron emission, ion induced electron emission, and surface charge accumulation would need to be modeled more accurately. And, because of the non-trivial shape, the grid construction procedure would have to be modified to allow unstructured grids to be handled.

- Plasma thrusters. To generate thrust efficiently, plasma thrusters use magnetic fields to confine the plasma Therefore, the code should expanded in order to solve the full set of Maxwell equations, and the particle mover should be modified to include Lorentz force. Depending on the plasma density, modifications similar to those discussed for plasma etchers may be required as well.

In short, there many other plasma applications, with each requiring some of the underlying assumptions and simplifications of the current code to be modified. These modifications are possible, but would be time consuming to implement, while also vastly decreasing the efficiency of the code. An alternative would be to couple this code to other code bases that include these processes. In such an implementation, the relevant assumptions could be switched on and off as appropriate, allowing efficient simulation of a wider range of plasma conditions. However, the different code bases should be selected with care. For succesefull results it should have either a similar internal implementation or a sophisticated application programming interface. 



\section{Chapter 9}

\section{Conclusions}

The goal of the present work was to build a numerical tool that can be used to analyse the behavior of EUV induced plasmas. The focus of our work was on the interaction between EUV induced plasma and the surface. Therefore, the model was designed to be able to provide spatiallyenergy-time and species resolved fluxes from the plasma to the surface. These fluxes would then be used elsewhere as input for simulating surface processes. However, for these purposes, a good understanding of the dynamics of the EUV plasma in the bulk and in the plasma sheath is absolutely necessary.

To accomplish this, the code must also contain, in addition to the right physics, relevant cross sections. Assembling a reasonably accurate cross-sections set, and testing the model implementation on available experimental data for swarm experiments was a necessary and time consuming step. However, this success established a good background to all further development.

The task of simulating and EUV induced plasma is complex, therefore, we have divided it into several sub tasks. The dynamics of the EUV plasma in the bulk have many similar features with other plasmas, predominantly electron dynamics and collisions. Therefore, first, it was necessary to check whether the model predicts the right plasma density in a discharge without EUV.

For this purpose we compared the model predictions and experimental measurements of a low density transient plasma. The initial goal was to compare the model predicted plasma density the measured densities, obtained from the analysis of probe data. However, the significant disagreement between the two lead us to analyse the applicability of the probe method itself and to directly compare measured and predicted probe responses.

As shown in chapter 5 , probe theories, especially in the case of a low density non-stationary plasma, should be used with caution. Our model results show that the probe response can appear to be normal, while the calculated density is incorrect. These calculations also show that, despite the distortion, probes can still be used. Depending on the applied probe theory, the density may be over estimated or under estimated. Thus, by applying a range of theories, one can estimate the uncertainty range of the plasma density.

Once the electron dynamics in the model were checked, we considered an experiment with EUV radiation. Fortunately, we were able to begin with an experiment that was specially designed to reduce the influence of the surface processes (e.g. photo electron emission). Therefore, these simulations focus on the dynamics of the ignition of the plasma by the EUV radiation.

However, we found, that, even for this case, the number of important parameters is still rather large. As discussed in the chapter 6, the cumulative uncertainties in the parameters are important. The uncertainty in the transmission spectrum of the SPF, and the possible error margin in the measured EUV source emission spectrum were found to significantly influence 
plasma densities, for instance. This sensitivity is due to the rapid increase of the absorption cross-section with decreasing photon energy, which greatly amplifies the contributions of VUV part of the spectrum. For example, even 1\% transmission of the SPF filter in the range of 20 $30 \mathrm{eV}$ has a significant effect on the EUV plasma formation in hydrogen.

It is also worth noting that other processes, such as electron-induced electron emission from surfaces can also play an important role, especially for low pressure cases, where the mean free path of the elections is comparable with the setup dimensions.

Finally, we have considered an experiment with EUV radiation incident on a sample (chapter 7). For this experiment, all the parameters and processes mentioned above are important. However, in this experiment, an external bias was used to attract the plasma to the sample. Interestingly, the applied bias greatly simplified the simulations, since the plasma evolution was controlled by the bias and not by the large number of poorly known parameters. We found good agreement between simulated and experimentally measured discharge characteristics for the setup. Using this agreement, the relative fluxes of different ion species were combined with the measured total flux to compute the species and energy resolved fluxes to the surface. These fluxes were used to quantitatively compare carbon etch rates between different EUV plasma conditions, and to compare carbon etching under EUV-induced plasma to etching under surface wave discharge plasmas.

It was found that for the intersecting range of energies there was good quantitative agreement (to within model and measurement uncertainties) between the etch rates observed in experiments with and without EUV. Therefore, there the role of the EUV for carbon etching is small, and, hence, the main etching mechanism is, most likely, chemical sputtering of carbon. 


\section{Acknowledgments}

The last five years of my life were very challenging, demanding and in the same time very interesting. First of all, I would like to thank Prof. Konstantin Koshelev who acquainted me with the FOM institute and helped me via numerous extremely wise pieces of advice. With his help and constant support I started my work in ISAN and later on participated in the Ph.D project in FOM institute Rijnhuizen. I am also very thankful to my promoter Fred Bijkerk for giving me an opportunity to enroll as a Ph.D student in the CP3E program and work on the problems of the EUV induced plasmas.

My supervisors: Wim Goedheer, Vladimir Ivanov, Dmitry Lopaev and Chris Lee were of great help in guiding my research during all these years. I am especially greateful to Chris for all time he spent reading my texts, for his brilliant comments. That made the process of writing papers and thesis a lot funnier.

This work would not have been possible without help and support from all the people involved in the experiments. I thank Oleg Yakushev, Vladimir Krivtsun, Dmitry Abramenko, Alexey Abrikosov, Alexey Zotovich, Sergey Zyryanov, Robert Gayasov and Ruud van der Horst for carrying out the experiments and endless discussions about the meaning of the experimental results.

Next I want to thank all the members of XUV group who I met during my Ph.D: especially Andrei Yakshin, Robert van de Kruijs and Erwin Zoethout. Great thanks go to Melanie van der Vlis-Kettmann and Jacqueline Emmerich-Nijenhuis for their support in dealing with Dutch administrative rules and regulations.

I also want to mention colleagues from the ASML team: Edgar Osorio, Kees de Meire, Andrei Yakunin and Maarten van Kampen. Thanks for providing me with your input and driving me to consider the industrial needs into the present work.

I could not help but mentioning Igor Makhotkin, Slava Medvedev, Alexander Dolgov, Sebastian Huber, Vladimir Kvon who accompanied me in a fun exploration of the Netherlands. I am especially grateful to Alexandra Elbakyan for the invaluable help with the literature search. 



\section{Curriculum vitae}

I was born on January 9, 1986 in Sverdlovsk, which is now called Ekaterinburg. My mother and father received their higher education there and moved to Sarov where I finished school. In the middle school I successfully participated in school physics competitions on the regional and federal state level and decided to continue my career in Physics. In 2003 I started the Bachelor Degree program in Moscow Institute of Physics and Technology (State University), MIPT, in the Faculty of Physics and Energy Problems and continued my Master Degree there in 2007. In 2009 I joined the Institute of Nuclear Research and studied quantum field theory embedded in a five dimensional space-time. My first scientific article was published in Phys. Rev. D and was called "Vector bosons escaping from the brane: $e^{+}+e^{-} \rightarrow \gamma+$ nothing". Nevertheless, later I decided to apply my knowledge in industrial issues and joined the ISAN scientific team, who acquainted me with FOM institute where I entered PhD program on the present topic. Since 2015, I have been working as a research scientist for RND-ISAN/ISTEQ. 



\section{Bibliography}

[1] Alfred Kwok-Kit. Wong. Resolution enhancement techniques in optical lithography. Bellingham, Wash.: SPIE Press, 2001. ISBN: 0-8194-3995-9.

[2] Yayi. Wei, Robert L. Brainard, and Society of Photo-optical Instrumentation Engineers. Advanced processes for 193-nm immersion lithography. Bellingham, Wash.: SPIE, 2009. ISBN: 978-0-8194-7843-6.

[3] S. Y. Chou, P. R. Krauss, and P. J. Renstrom. "Imprint Lithography with 25-Nanometer Resolution”. In: Science Vol. 272, No. 5258 (1996), pp. 85-87. DoI: 10.1126 / science. 272.5258 .85 .

[4] Masayoshi Esashi et al. "Development of massively parallel electron beam direct write lithography using active-matrix nanocrystalline-silicon electron emitter arrays”. In: $M i$ crosystems \& Nanoengineering Vol. 1 (2015), p. 15029. DoI: 10.1038/micronano. 2015. 29.

[5] Vivek Bakshi. EUV lithography. Bellingham, Wash.; Hoboken, NJ: SPIE Press ; John Wiley, 2009. ISBN: 978-1-61583-717-5.

[6] Theodore E. Madey et al. "Surface phenomena related to mirror degradation in extreme ultraviolet (EUV) lithography”. In: Applied Surface Science Vol. 253, No. 4 (2006), pp. 16911708. DoI: $10.1016 / j$.apsusc. 2006.04.065.

[7] Jeromy Hollenshead and Leonard Klebanoff. "Modeling radiation-induced carbon contamination of extreme ultraviolet optics". In: fournal of Vacuum Science \& Technology B: Microelectronics and Nanometer Structures Vol. 24, No. 1 (2006), pp. 64-82. DoI: $10.1116 /$ 1.2140005 .

[8] S. B. Hill et al. "Accelerated lifetime metrology of EUV multilayer mirrors in hydrocarbon environments". In: Emerging Lithographic Technologies XII. Emerging Lithographic Technologies XII. Ed. by Frank M. Schellenberg. Vol. 6921. San Jose, CA, USA: SPIE, 2008, pp. 692117-11.

[9] Yasushi Nishiyama et al. "Carbon contamination of EUV mask: film characterization, impact on lithographic performance, and cleaning”. In: Emerging Lithographic Technologies XII. Emerging Lithographic Technologies XII. Ed. by Frank M. Schellenberg. Vol. 6921. San Jose, CA, USA: SPIE, 2008, pp. 692116-10. 
[10] Takahiro Nakayama et al. "Analysis of carbon deposition on multilayer mirrors by using two different beamlines”. In: Alternative Lithographic Technologies. Alternative Lithographic Technologies. Ed. by Frank M. Schellenberg and Bruno M. La Fontaine. Vol. 7271. San Jose, CA, USA: SPIE, 2009, 72713P-8.

[11] S. B. Hill et al. "Complex species and pressure dependence of intensity scaling laws for contamination rates of EUV optics determined by XPS and ellipsometry". In: Extreme Ultraviolet (EUV) Lithography. Extreme Ultraviolet (EUV) Lithography. Ed. by Bruno M. La Fontaine. Vol. 7636. San Jose, California, USA: SPIE, 2010, 76360E-10.

[12] Kevin Kemp and Stefan Wurm. "EUV lithography". In: Comptes Rendus Physique Vol. 7, No. 8 (2006), pp. 875-886. DoI: 10.1016/j . crhy . 2006.10.002.

[13] Burn J. Lin. "Sober view on extreme ultraviolet lithography". In: fournal of Microlithography, Microfabrication, and Microsystems Vol. 5, No. 3 (2006), pp. 033005-12.

[14] V. Y. Banine. "EUV Lithography: today and tomorrow". In: Future Trends in Microelectronics: Frontiers and Innovations. 2013, pp. 120-132.

[15] Vivek Bakshi. EUV sources for lithography. Bellingham, Wash.: SPIE Press, 2006. IsBN: 978-1-61583-716-8.

[16] V. Y. Banine, K. N. Koshelev, and G. H. P. M. Swinkels. "Physical processes in EUV sources for microlithography”. In: Journal of Physics D: Applied Physics Vol. 44, No. 25 (2011), p. 253001. DOI: 10.1088/0022-3727/44/25/253001.

[17] David C. Brandt et al. "LPP EUV source readiness for NXE 3300B". In: ed. by Obert R. Wood and Eric M. Panning. 2014, p. 90480C. DoI: 10.1117/12.2048184.

[18] Berg et al. "A plan to measure EUV resist contamination in the presence of hydrogen". In: International Workshop on EUV Lithography. Maui, Hawaii, 2015.

[19] J. P. Verboncoeur, A. B. Langdon, and N. T. Gladd. "An object-oriented electromagnetic PIC code”. In: Computer Physics Communications. Particle Simulation Methods Vol. 87, No. 1-2 (1995), pp. 199-211. DOI: 10.1016/0010-4655(94)00173-Y.

[20] S. F. Biagi. "A multiterm Boltzmann analysis of drift velocity, diffusion, gain and magneticfield effects in argon-methane-water-vapour mixtures". In: Nuclear Instruments and Methods in Physics Research Section A: Accelerators, Spectrometers, Detectors and Associated Equipment Vol. 283, No. 3 (1989), pp. 716-722. Dor: 10 . 1016/0168-9002 (89) 91446-0.

[21] G. J. M. Hagelaar and L. C. Pitchford. "Solving the Boltzmann equation to obtain electron transport coefficients and rate coefficients for fluid models”. In: Plasma Sources Science and Technology Vol. 14, No. 4 (2005), p. 722. DoI: 10.1088/0963-0252/14/4/011.

[22] M. H. L. van der Velden et al. "Particle-in-cell Monte Carlo simulations of an extreme ultraviolet radiation driven plasma”. In: Physical Review E Vol. 73, No. 3 (2006), p. 036406. DOI: 10.1103/PhysRevE.73.036406.

[23] R. C. Wieggers et al. "Plasma-induced damage of multilayer coatings in EUVL". In: Proceedings of SPIE. Damage to VUV, EUV, and X-ray Optics. Prague, Czech Republic, 2007, pages. DOI: $10.1117 / 12.724889$. 
[24] Ivanov M. F. and Galburg V. A. Numerical simulations of gas and plasma dynamics (in russian: Численное моделирование динамики газов и плазмы методами частиц). MIPT, 2000. 168 pp. ISBN: 5-7417-0141-8.

[25] Yu. P. Raizer. Gas discharge physics. Berlin; New York: Springer-Verlag, 1991. ISBN: 3-54019462-2.

[26] H. Ralph Lewis. "Energy-conserving numerical approximations for Vlasov plasmas". In: Journal of Computational Physics Vol. 6, No. 1 (1970), pp. 136-141. DoI: 10.1016/00219991 (70)90012-4.

[27] A. Bruce Langdon. ““Energy-conserving” plasma simulation algorithms”. In: fournal of Computational Physics Vol. 12, No. 2 (1973), pp. 247-268. DoI: 10.1016/S0021-9991 (73) 80014-2.

[28] Charles K Birdsall and A. Bruce Langdon. Plasma physics via computer simulation. New York: McGraw-Hill, 1985. ISBN: 0-07-005371-5 978-0-07-005371-7.

[29] R. P. Fedorenko. "Iterative Methods for Elliptic Difference Equations". In: Russian Mathematical Surveys Vol. 28 (1973), pp. 129-195. DOI: 10.1070/RM1973v028n02ABEH001542.

[30] W Hackbusch. Multi-grid methods and applications. Berlin; New York: Springer-Verlag, 1985. ISBN: 0-387-12761-5.

[31] William L Briggs, Van Emden Henson, and S. F McCormick. A multigrid tutorial. Philadelphia, PA: Society for Industrial and Applied Mathematics, 2000. ISBN: 978-0-89871-462-3.

[32] U. Trottenberg. Multigrid. In collab. with C. W. Oosterlee and Anton Schüller. San Diego: Academic Press, 2001. 631 pp. ISBN: 0-12-701070-X.

[33] Gundolf Haase and Ulrich Langer. "Multigrid methods: from geometrical to algebraic versions”. In: Modern methods in scientific computing and applications. Springer, 2002, pp. 103-153.

[34] M. Brezina et al. "Algebraic Multigrid Based on Element Interpolation (AMGe)". In: SIAM Journal on Scientific Computing Vol. 22, No. 5 (2001), pp. 1570-1592. DOI: 10 . 1137 / S1064827598344303.

[35] V. Henson and P. Vassilevski. "Element-Free AMGe: General Algorithms for Computing Interpolation Weights in AMG”. In: SIAM Journal on Scientific Computing Vol. 23, No. 2 (2001), pp. 629-650. DOI: 10.1137/S1064827500372997.

[36] Y. M Chung et al. "Dissociative photoionization of $\mathrm{H}_{2}$ from 18 to $124 \mathrm{eV}$ ". In: The fournal of Chemical Physics Vol. 99, No. 2 (1993), pp. 885-889. DoI: doi:10.1063/1.465352.

[37] B. V. Yakshinskiy et al. "Carbon accumulation and mitigation processes, and secondary electron yields of ruthenium surfaces”. In: Proceedings of SPIE. Emerging Lithographic Technologies XI. San Jose, CA, USA, 2007, pages. DOI: 10.1117/12.711785.

[38] B Yakshinskiy et al. "DIET processes on ruthenium surfaces related to extreme ultraviolet lithography (EUVL)”. In: Surface Science Vol. 602, No. 20 (2008), pp. 3220-3224. DoI: 10 . $1016 / j$.susc. 2007.10 .055$. 
[39] Burton L. Henke, Jerel A. Smith, and David T. Attwood. "0.1-10-keV x-ray-induced electron emissions from solids-Models and secondary electron measurements". In: fournal of Applied Physics Vol. 48 (1977), p. 1852. DOI: 10.1063/1. 323938.

[40] David R Lide. CRC handbook of chemistry and physics, 2003-2004: a ready-reference book of chemical and physical data. CRC Press, 2003. IsBN: 0-8493-0484-9.

[41] H. R. Skullerud. "The stochastic computer simulation of ion motion in a gas subjected to a constant electric field”. In: Journal of Physics D: Applied Physics Vol. 1, No. 11 (1968), p. 1567. DOI: $10.1088 / 0022-3727 / 1 / 11 / 423$.

[42] Kenichi Nanbu. "Simple Method to Determine Collisional Event in Monte Carlo Simulation of Electron-Molecule Collision”. In: fapanese fournal of Applied Physics Vol. 33 (Part 1, No. 8 1994), pp. 4752-4753. DOI: 10.1143/J JAP. 33.4752.

[43] H. Tawara et al. "Cross Sections and Related Data for Electron Collisions with Hydrogen Molecules and Molecular Ions”. In: Journal of Physical and Chemical Reference Data Vol. 19, No. 3 (1990), p. 617. DoI: 10.1063/1.555856.

[44] Jung-Sik Yoon et al. "Cross Sections for Electron Collisions with Hydrogen Molecules". In: Journal of Physical and Chemical Reference Data Vol. 37, No. 2 (2008), p. 913. Dor: $10.1063 / 1.2838023$.

[45] R. K. Janev, D. Reiter, and U. Samm. Collision Processes in Low-Temperature Hydrogen Plasmas. 4105. FZ-Juelich, 2003.

[46] M. J. Brunger and S. J. Buckman. "Electron-molecule scattering cross-sections. I. Experimental techniques and data for diatomic molecules". In: Physics reports Vol. 357, No. 3 (2002), pp. 215-458.

[47] S. J. Buckman and A. V. Phelps. "Vibrational excitation of $\mathrm{D}_{2}$ by low energy electrons". In: The fournal of Chemical Physics Vol. 82, No. 11 (1985), p. 4999. Dor: 10.1063/1.448673.

[48] A. V. Phelps. "Cross Sections and Swarm Coefficients for $\mathrm{H}^{+}, \mathrm{H}_{2}^{+}, \mathrm{H}_{3}^{+}, \mathrm{H}, \mathrm{H}_{2}$, and $\mathrm{H}^{-}$in $\mathrm{H}_{2}$ for Energies from $0.1 \mathrm{eV}$ to $10 \mathrm{keV}$ ". In: Journal of Physical and Chemical Reference Data Vol. 19, No. 3 (1990), p. 653. DoI: 10.1063/1.555858.

[49] S. F. Biagi. "Monte Carlo simulation of electron drift and diffusion in counting gases under the influence of electric and magnetic fields". In: Nuclear Instruments and Methods in Physics Research Section A: Accelerators, Spectrometers, Detectors and Associated Equipment Vol. 421, No. 1 (1999), pp. 234-240.

[50] A. V. Phelps. "Energetic ion, atom, and molecule reactions and excitation in low-current $\mathrm{H}_{2}$ discharges: Model”. In: Physical Review E Vol. 79, No. 6 (2009), p. 066401. Dor: 10 . 1103/PhysRevE.79.066401.

[51] L. L. Alves et al. "Comparisons of sets of electron-neutral scattering cross sections and swarm parameters in noble gases: II. Helium and neon”. In: Journal of Physics D: Applied Physics Vol. 46, No. 33 (2013), p. 334002. DOI: 10.1088/0022-3727/46/33/334002.

[52] Z. Lj. Petrović et al. "Kinetic phenomena in charged particle transport in gases, swarm parameters and cross section data". In: Plasma Sources Science and Technology Vol. 16, No. 1 (2007), S1-S12. DoI: 10 . 1088/0963-0252/16/1/S01. 
[53] Z. Lj. Petrović et al. "Measurement and interpretation of swarm parameters and their application in plasma modelling”. In: Journal of Physics D: Applied Physics Vol. 42, No. 19 (2009), p. 194002. DOI: 10.1088/0022-3727/42/19/194002.

[54] J. Dutton. "A survey of electron swarm data”. In: Gournal of Physical and Chemical Reference Data Vol. 4, No. 3 (1975), pp. 577-856. Dor: 10.1063/1. 555525.

[55] E. Graham et al. "Mobilities and longitudinal diffusion coefficients of mass-identified hydrogen ions in $\mathrm{H}_{2}$ and deuterium ions in $\mathrm{D}_{2}$ gas". In: The fournal of Chemical Physics Vol. 59, No. 7 (1973), pp. 3477-3481. DoI: doi:10.1063/1.1680505.

[56] R. F. Stebbings and B. G. Lindsay. "Comment on the accuracy of absolute electron-impact ionization cross sections for molecules”. In: The fournal of Chemical Physics Vol. 114, No. 10 (2001), pp. 4741-4743. DoI: doi:10.1063/1.1346641.

[57] T. W. Shyn, W. E. Sharp, and Y.-K. Kim. "Doubly differential cross sections of secondary electrons ejected from gases by electron impact: 25-250 eV on $\mathrm{H}_{2}$ ”. In: Physical Review A Vol. 24, No. 1 (1981), pp. 79-88. DoI: 10.1103/PhysRevA.24.79.

[58] M. E. Rudd et al. "Doubly differential electron-production cross sections for 200-1500eV e $+\mathrm{H}_{2}$ collisions”. In: Physical Review A Vol. 47, No. 3 (1993), pp. 1866-1873. DoI: 10.1103/PhysRevA.47.1866.

[59] H. Ehrhardt et al. "Resonance Scattering of Slow Electrons from $\mathrm{H}_{2}$ and $\mathrm{CO}$ Angular Distributions”. In: Physical Review Vol. 173, No. 1 (1968), p. 222. DoI: 10.1103/PhysRev . 173. 222.

[60] J. P. England, M. T. Elford, and R. W. Crompton. "A Study of the Vibrational Excitation of $\mathrm{H}_{2}$ by Measurements of the Drift Velocity of Electrons in $\mathrm{H}_{2}-\mathrm{Ne}$ Mixtures”. In: Aust. f. Phys. Vol. 41, No. 4 (1988), pp. 573-586.

[61] R. H. Garvey, H. S. Porter, and A. E. S. Green. "Relativistic yield spectra for $\mathrm{H}_{2}$ ”. In: fournal of Applied Physics Vol. 48, No. 10 (1977), pp. 4353-4359. Dor: 10.1063/1. 323427.

[62] T. Šimko et al. "Computer simulations of $\mathrm{H}^{+}$and $\mathrm{H}_{3}^{+}$transport parameters in hydrogen drift tubes”. In: Physical Review E Vol. 56, No. 5 (1997), pp. 5908-5919. DoI: 10 . 1103/ PhysRevE.56.5908.

[63] B. L. Peko and R. L. Champion. "Total cross sections for low energy collisions of $\mathrm{H}_{3}^{+}$with molecular hydrogen and rare gases”. In: The fournal of Chemical Physics Vol. 107 (1997), p. 1156. DOI: $10.1063 / 1.474462$.

[64] M. S. Mokrov and Yu P. Raizer. "Monte Carlo method for finding the ionization and secondary emission coefficients and I-V characteristic of a Townsend discharge in hydrogen”. In: Technical Physics Vol. 53, No. 4 (2008), pp. 436-444. Dor: 10 .1134/S1063784208040075.

[65] Predrag S Krstic and David R Schultz. "Elastic and vibrationally inelastic slow collisions: $\mathrm{H}+\mathrm{H}_{2}, \mathrm{H}^{+}+\mathrm{H}_{2}$ ”. In: fournal of Physics B: Atomic, Molecular and Optical Physics Vol. 32, No. 10 (1999), pp. 2415-2431. DOI: 10.1088/0953-4075/32/10/310.

[66] Homer D. Hagstrum. "Theory of Auger Ejection of Electrons from Metals by Ions". In: Physical Review Vol. 96, No. 2 (1954), pp. 336-365. DoI: 10.1103/PhysRev .96. 336. 
[67] L. M. Kishinevsky. "Estimation of electron potential emission yield dependence on metal and ion parameters”. In: Radiation Effects Vol. 19, No. 1 (1973), pp. 23-27. DoI: 10.1080 / 00337577308232211.

[68] G. Lakits et al. "Threshold of ion-induced kinetic electron emission from a clean metal surface”. In: Physical Review A Vol. 42, No. 9 (1990). Dor: 10.1103/PhysRevA . 42.5780.

[69] H Winter et al. "Slow-ion induced electron emission from clean metal surfaces: "Subthreshold kinetic emission" and "potential excitation of plasmons"”. In: Nuclear Instruments and Methods in Physics Research B Vol. 182 (2001), pp. 15-22. DoI: 10 . 1016/S0168583X (01) 00649-8.

[70] Annemie Bogaerts and Renaat Gijbels. "Hybrid Monte Carlo-fluid modeling network for an argon/hydrogen direct current glow discharge". In: Spectrochimica Acta Part B: Atomic Spectroscopy Vol. 57, No. 6 (2002), pp. 1071-1099. DOI: 10 . 1016/S0584-8547 (02) 00047-2.

[71] H. Winter, F. Aumayr, and G. Lakits. "Recent advances in understanding particle-induced electron emission from metal surfaces". In: Nuclear Instruments and Methods in Physics Research Section B: Beam Interactions with Materials and Atoms Vol. 58, No. 3 (1991), pp. 301-308. DoI: 10.1016/0168-583X (91) 95859-C.

[72] R. A. Baragiola, E. V. Alonso, and A. Oliva Florio. "Electron emission from clean metal surfaces induced by low-energy light ions”. In: Physical Review B Vol. 19, No. 1 (1979), p. 121. Dor: 10.1103/PhysRevB.19.121.

[73] C. D. Child. "Discharge From Hot CaO". In: Physical Review (Series I) Vol. 32, No. 5 (1911), pp. 492-511. DoI: 10.1103/PhysRevSeriesI.32.492.

[74] Irving Langmuir. "The Effect of Space Charge and Initial Velocities on the Potential Distribution and Thermionic Current between Parallel Plane Electrodes”. In: Physical Review Vol. 21, No. 4 (1923), pp. 419-435. DoI: 10.1103/PhysRev .21. 419.

[75] I. H. Hutchinson. Principles of Plasma Diagnostics. Cambridge University Press, 2005. 460 pp. ISBN: 978-0-521-67574-1.

[76] Francis F. Chen. "Langmuir probe diagnostics". In: Mini-Course on Plasma Diagnostics, IEEE-ICOPS Meeting, Jeju, Korea. 2003.

[77] V Agarwala and T Fort. "Work function changes during low pressure oxidation of aluminum at room temperature”. In: Surface Science Vol. 45 (1974), pp. 470-482. Dor: 10 . 1016/0039-6028(74)90183-6.

[78] Edward Delarosa Sosa. "The electron emission characteristics of aluminum, molybdenum and carbon nanotubes studied by field emission and photoemission". University of North Texas, 2002.

[79] Hanspeter Helm. "Measurement of the ionization potential of triatomic hydrogen". In: Physical Review A Vol. 38, No. 7 (1988), pp. 3425-3429. Dor: 10 . 1103/PhysRevA . 38. 3425.

[80] F. Taccogna, S. Longo, and M. Capitelli. "Ion-Neutral Collision Effects in Langmuir Probe Theory”. In: Contributions to Plasma Physics Vol. 48, No. 5-7 (2008), pp. 509-514. DoI: 10.1002/ctpp. 200810082. 
[81] James G. Laframboise. Theory of spherical and cylindrical Langmuir probes in a collisionless, Maxwellian plasma at rest. UTIAS Report NO. 100. University of Toronto, Institute for Aerospace studies: DTIC Document, 1966.

[82] J E Allen, R L F Boyd, and P Reynolds. "The Collection of Positive Ions by a Probe Immersed in a Plasma”. In: Proceedings of the Physical Society. Section B Vol. 70, No. 3 (1957), pp. 297-304. DOI: 10.1088/0370-1301/70/3/303.

[83] Francis F. Chen. "Numerical Computations for Ion Probe Characteristics in a Collisionless Plasma”. In: J. Nucl. Energy Vol. Pt. C 7, No. 47 (1965).

[84] Talbot L and Chou YS. "Langmuir probe response in the transition regime". In: Rarefied Gas Dynamics Vol. 2 (Suppl 5 1969), pp. 1723-1737.

[85] M. Tichý et al. "A Collisional Model of the Positive Ion Collection by a Cylindrical Langmuir Probe”. In: Contributions to Plasma Physics Vol. 34, No. 1 (1994), pp. 59-68. DoI: $10.1002 /$ ctpp. 2150340108.

[86] O. Chudáček et al. "Langmuir Probe Determination of Charged Particle Number Density in a Flowing Afterglow Plasma”. In: Contributions to Plasma Physics Vol. 35, No. 6 (1995), pp. 503-516. DOI: 10.1002/ctpp. 2150350605.

[87] Felipe Iza and Jae Koo Lee. "Particle-in-cell simulations of planar and cylindrical Langmuir probes: Floating potential and ion saturation current". In: Journal of Vacuum Science \& Technology A Vol. 24, No. 4 (2006), pp. 1366-1372. DoI: 10.1116/1.2187991.

[88] J M Díaz-Cabrera et al. "Experimental radial motion to orbital motion transition in cylindrical Langmuir probes in low pressure plasmas”. In: Plasma Sources Science and Technology Vol. 24, No. 2 (2015), p. 025026. Dor: 10.1088/0963-0252/24/2/025026.

[89] David J. Davis et al. “Toward the In Situ Remediation of Carbon Deposition on Ru-Capped Multilayer Mirrors Intended for EUV Lithography: Exploiting the Electron-Induced Chemistry”. In: The fournal of Physical Chemistry C Vol. 111, No. 33 (2007), pp. 12165-12168. DOI: $10.1021 /$ jp074766y.

[90] R. M. van der Horst et al. "Exploring the temporally resolved electron density evolution in extreme ultra-violet induced plasmas". In: Journal of Physics D: Applied Physics Vol. 47, No. 30 (2014), p. 302001. DOI: 10.1088/0022-3727/47/30/302001.

[91] S. Gundermann et al. "Microwave diagnostics of the electron density in molecular mixture plasmas”. In: Contributions to Plasma Physics Vol. 41, No. 1 (2001), pp. 45-60.

[92] van der MHL (Marc) Velden et al. "Radiation generated plasmas:a challenge in modern lithography”. Technische Universiteit Eindhoven, 2008.

[93] A Dolgov et al. "Extreme ultraviolet (EUV) source and ultra-high vacuum chamber for studying EUV-induced processes”. In: Plasma Sources Science and Technology Vol. 24, No. 3 (2015), p. 035003. DOI: 10.1088/0963-0252/24/3/035003.

[94] J.W. Gallagher et al. "Absolute Cross Sections for Molecular Photoabsorption, Partial Photoionization, and Ionic Photofragmentation Processes”. In: fournal of Physical and Chemical Reference Data Vol. 17, No. 1 (1987), pp. 9-153. DoI: 10.1063/1.555821. 
[95] R. G. Houlgate et al. "The angular distribution of the $3 p$ electrons and the partial cross section of the 3s electrons of argon from threshold to $70 \mathrm{eV}$ ”. In: Fournal of Electron Spectroscopy and Related Phenomena Vol. 9, No. 2 (1976), pp. 205-209. DoI: 10 . 1016/03682048 (76) 81030-4.

[96] K. T. Taylor. "Photoelectron angular-distribution beta parameters for neon and argon". In: Journal of Physics B: Atomic and Molecular Physics Vol. 10, No. 18 (1977), p. L699.

[97] James A. R. Samson and G. N. Haddad. "Total photoabsorption cross sections of H2 from 18 to $113 \mathrm{eV}$ ”. In: fournal of the Optical Society of America B Vol. 11, No. 2 (1994), pp. 277279. DOI: $10.1364 / J O S A B .11 .000277$.

[98] J. A.R. Samson et al. "Recent progress on the measurement of absolute atomic photoionization cross sections”. In: Le fournal de Physique IV Vol. 01 (C1 1991), pages. Dor: 10 . 1051/jp4:1991113.

[99] W. F. Chan et al. "Absolute optical oscillator strengths for the electronic excitation of atoms at high resolution. III. The photoabsorption of argon, krypton, and xenon". In: Physical Review A Vol. 46, No. 1 (1992), pp. 149-171. DoI: 10.1103/PhysRevA .46.149.

[100] J. B. West and G. V. Marr. "The Absolute Photoionization Cross Sections of Helium, Neon, Argon and Krypton in the Extreme Vacuum Ultraviolet Region of the Spectrum”. In: Proceedings of the Royal Society A: Mathematical, Physical and Engineering Sciences Vol. 349, No. 1658 (1976), pp. 397-421. DoI: 10.1098/rspa.1976.0081.

[101] Joseph Berkowitz. Atomic and molecular photoabsorption: absolute total cross sections. Academic Press, 2001.

[102] D. M. P. Holland et al. "Multiple photoionisation in the rare gases from threshold to 280 eV”. In: Journal of Physics B: Atomic and Molecular Physics Vol. 12, No. 15 (1979), p. 2465.

[103] G. D. Hobbs and J. A. Wesson. "Heat flow through a Langmuir sheath in the presence of electron emission”. In: Plasma Physics Vol. 9, No. 1 (1967), p. 85.

[104] V. Baglin et al. The secondary electron yield of technical materials and its variation with surface treatments. LHC-Project-Report-433. 2000.

[105] Yinghong Lin and David C. Joy. "A new examination of secondary electron yield data". In: Surface and Interface Analysis Vol. 37, No. 11 (2005), pp. 895-900. DOI: 10.1002/sia. 2107.

[106] M. Belhaj et al. Electron emission at very low electron impact energy: Experimental and Monte-Carlo results. 2013. DOI: 10.5170/CERN-2013-002.137.

[107] P. H. Dawson. "Secondary Electron Emission Yields of some Ceramics”. In: fournal of Applied Physics Vol. 37, No. 9 (1966), p. 3644. Dor: 10.1063/1.1708934.

[108] T. Tondu, M. Belhaj, and V. Inguimbert. "Electron-emission yield under electron impact of ceramics used as channel materials in Hall-effect thrusters". In: fournal of Applied Physics Vol. 110, No. 9 (2011), p. 093301. DoI: 10.1063/1.3653820.

[109] R. H. Day et al. "Photoelectric quantum efficiencies and filter window absorption coefficients from $20 \mathrm{eV}$ to $10 \mathrm{KeV}$ ”. In: Journal of Applied Physics Vol. 52, No. 11 (1981), pp. 6965-6973. DOI: 10.1063/1.328653. 
[110] John Hornbeck. "The Drift Velocities of Molecular and Atomic Ions in Helium, Neon, and Argon”. In: Physical Review Vol. 84, No. 4 (1951), pp. 615-620. Dor: 10.1103/PhysRev . 84.615 .

[111] Rainer Johnsen and Manfred A. Biondi. "Mobilities of doubly charged rare-gas ions in their parent gases”. In: Physical Review A Vol. 18, No. 3 (1978), pp. 989-995. DoI: 10 . 1103/PhysRevA.18.989.

[112] Bibliography of Electron and Photon Cross Sections with Atoms. URL: http://www .nifs . ac.jp/report/nifs-data072.html.

[113] A.V. Phelps webapge. URL: http://jilawww.colorado.edu/ avp/.

[114] R. Rejoub, B. G. Lindsay, and R. F. Stebbings. "Determination of the absolute partial and total cross sections for electron-impact ionization of the rare gases". In: Physical Review A Vol. 65, No. 4 (2002), p. 042713. DoI: 10.1103/PhysRevA.65. 042713.

[115] Brent R. Yates and Murtadha A. Khakoo. "Near-threshold electron-impact doubly differential cross sections for the ionization of argon and krypton". In: Physical Review A Vol. 83, No. 4 (2011), p. 042712. DoI: 10.1103/PhysRevA.83. 042712.

[116] A. Fiala, L. C. Pitchford, and J. P. Boeuf. "Two-dimensional, hybrid model of low-pressure glow discharges”. In: Physical Review E Vol. 49, No. 6 (1994), p. 5607.

[117] H. Deutsch et al. "Calculated cross sections for the electron-impact ionization of metastable atoms”. In: Journal of Physics B: Atomic, Molecular and Optical Physics Vol. 32, No. 17 (1999), p. 4249.

[118] A. V. Phelps. "The application of scattering cross sections to ion flux models in discharge sheaths". In: Journal of Applied Physics Vol. 76, No. 2 (1994), p. 747. DOI: 10 . 1063 / 1. 357820.

[119] Kazuhiko Okuno. "Charge Transfer of $\mathrm{Ar}^{+2}$ and $\mathrm{Kr}^{+2}$ in Their Own Gases Studied by the Beam Guide Technique”. In: Journal of the Physical Society of Japan Vol. 55, No. 5 (1986), pp. 1504-1515. DOI: 10.1143/JPSJ.55.1504.

[120] R. M. van der Horst et al. "Exploring the electron density in plasmas induced by extreme ultraviolet radiation in argon". In: Journal of Physics D: Applied Physics Vol. 48, No. 28 (2015), p. 285203. DOI: 10.1088/0022-3727/48/28/285203.

[121] R M van der Horst et al. "Dynamics of the spatial electron density distribution of EUVinduced plasmas". In: Journal of Physics D: Applied Physics Vol. 48, No. 43 (2015), p. 432001. DOI: $10.1088 / 0022-3727 / 48 / 43 / 432001$.

[122] K. Boller et al. "Investigation of carbon contamination of mirror surfaces exposed to synchrotron radiation”. In: Nuclear Instruments and Methods in Physics Research Vol. 208, No. 1-3 (1983), pp. 273-279. DoI: 10 .1016/0167-5087 (83) 91134-1.

[123] Jeromy Hollenshead and Leonard Klebanoff. "Modeling extreme ultraviolet $/ \mathrm{H}_{2} \mathrm{O}$ oxidation of ruthenium optic coatings". In: Journal of Vacuum Science \& Technology B: Microelectronics and Nanometer Structures Vol. 24, No. 1 (2006), pp. 118-130. DoI: 10.1116/1. 2150225. 
[124] H Shin et al. "Reflectivity degradation of grazing-incident EUV mirrors by EUV exposure and carbon contamination”. In: Microelectronic Engineering Vol. 86, No. 1 (2009), pp. 99105. DOI: $10.1016 / j$. mee.2008.10.009.

[125] C. Hopf, A. von Keudell, and W. Jacob. "Chemical sputtering of hydrocarbon films". In: fournal of Applied Physics Vol. 94, No. 4 (2003), pp. 2373-2380. DoI: doi : 10 . 1063/1. 1594273.

[126] Jürgen Küppers. "The hydrogen surface chemistry of carbon as a plasma facing material". In: Surface Science Reports Vol. 22, No. 7-8 (1995), pp. 249-321. DoI: 10 . 1016 / 0167 5729 (96) 80002-1.

[127] Shengguang Liu et al. "A general model for chemical erosion of carbon materials due to low-energy H $\mathrm{H}^{+}$impact”. In: Journal of Applied Physics Vol. 108, No. 7 (2010), p. 073302. DOI: $10.1063 / 1$. 3485821 .

[128] Bhavin N. Jariwala, Cristian V. Ciobanu, and Sumit Agarwal. "Atomic hydrogen interactions with amorphous carbon thin films”. In: Journal of Applied Physics Vol. 106, No. 7 (2009), p. 073305. DOI: 10.1063/1.3238305.

[129] A. Dolgov et al. "Comparison of $\mathrm{H}_{2}$ and He carbon cleaning mechanisms in extreme ultraviolet induced and surface wave discharge plasmas”. In: fournal of Physics D: Applied Physics Vol. 47, No. 6 (2014), p. 065205. DoI: 10.1088/0022-3727/47/6/065205.

[130] A. Dunaevsky, Y. Raitses, and N. J. Fisch. "Secondary electron emission from dielectric materials of a Hall thruster with segmented electrodes”. In: Physics of Plasmas Vol. 10, No. 6 (2003), p. 2574. DOI: 10.1063/1.1568344.

[131] H. A. Fowler and H. E. Farnsworth. "Reflection of Very Slow Electrons". In: Physical Review Vol. 111, No. 1 (1958), pp. 103-112. Dor: 10.1103/PhysRev.111.103.

[132] Yasunori Yamamura and Hiro Tawara. "Energy dependence of ion-induced sputtering yields from monatomic solids at normal incidence". In: Atomic Data and Nuclear Data Tables Vol. 62, No. 2 (1996), pp. 149-253. Dor: 10.1006/adnd.1996.0005.

[133] A. Gao et al. "Defect formation in single layer graphene under extreme ultraviolet irradiation”. In: Applied Surface Science Vol. 317 (2014), pp. 745-751. Dor: 10 . 1016/ j . apsusc . 2014.08 .177$.

[134] Y. Socol et al. "Compact 13.5-nm free-electron laser for extreme ultraviolet lithography". In: Physical Review Special Topics - Accelerators and Beams Vol. 14, No. 4 (2011), p. 040702. DoI: 10.1103/PhysRevSTAB . 14.040702. 